\title{
Surgical options to treat heart failure patients
}

Citation for published version (APA):

Bolotin, G. (2001). Surgical options to treat heart failure patients: monitoring, analysis, and development of skeletal muscle operations. [Doctoral Thesis, Maastricht University]. Datawyse / Universitaire Pers Maastricht. https://doi.org/10.26481/dis.20010504gb

Document status and date:

Published: 01/01/2001

DOI:

10.26481/dis.20010504gb

Document Version:

Publisher's PDF, also known as Version of record

\section{Please check the document version of this publication:}

- A submitted manuscript is the version of the article upon submission and before peer-review. There can be important differences between the submitted version and the official published version of record.

People interested in the research are advised to contact the author for the final version of the publication, or visit the DOI to the publisher's website.

- The final author version and the galley proof are versions of the publication after peer review.

- The final published version features the final layout of the paper including the volume, issue and page numbers.

Link to publication

\footnotetext{
General rights rights.

- You may freely distribute the URL identifying the publication in the public portal. please follow below link for the End User Agreement:

www.umlib.nl/taverne-license

Take down policy

If you believe that this document breaches copyright please contact us at:

repository@maastrichtuniversity.nl

providing details and we will investigate your claim.
}

Copyright and moral rights for the publications made accessible in the public portal are retained by the authors and/or other copyright owners and it is a condition of accessing publications that users recognise and abide by the legal requirements associated with these

- Users may download and print one copy of any publication from the public portal for the purpose of private study or research.

- You may not further distribute the material or use it for any profit-making activity or commercial gain

If the publication is distributed under the terms of Article $25 \mathrm{fa}$ of the Dutch Copyright Act, indicated by the "Taverne" license above, 
SURGICAL OPTIONS TO TREAT

HEART FAILURE PATIENTS 
(C) G. Bolotin, Maastricht 2001

ISBN 9052783039

Vormgeving en druk: Datawyse | Universitaire Pers Maastricht 


\title{
SURGICAL OPTIONS TO TREAT HEART FAILURE PATIENTS
}

\author{
Monitoring, analysis, and development \\ of skeletal muscle operations
}

\author{
Proefschrift \\ ter verkrijging van de graad van doctor \\ aan de Universiteit Maastricht, \\ op gezag van de Rector Magnificus, \\ Prof.dr. A.C. Nieuwenhuijzen Kruseman, \\ volgens het besluit van het College van Decanen, \\ in het openbaar te verdedigen \\ op vrijdag 4 mei 2001 om 12.00 uur
}

door

Gil Bolotin

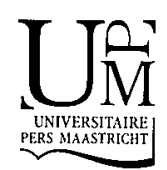




\section{Promotor}

Prof.dr. B. Mochtar

\section{Co-promotor}

Dr.ir. F. H. van der Veen

\section{Beoordelingscommissie}

Prof.dr. M.J.A.P. Daemen (voorzitter)

Prof.dr.ir. T. Arts

Prof.dr. M. Brouwer (UMC Nijmegen)

Prof.dr. M. Jacobs

Dr. V. van Ommen 
Dedicated to the memory of my dear friend, Eyal Prass 


\section{Contents}

List of abbreviations

CHAPTER 1

Introduction

CHAPTER 2

Acute and chronic heart dilatation model induced in goats by carotid jugular A-V shunt

CHAPTER 3

Peri-operative hemodynamic and geometrical changes of the left ventricle during cardiomyoplasty in goats with dilated left ventricle

CHAPTER 4

Early effects of cardiomyoplasty in a goat model of ventricular dilatation

CHAPTER 5

The use of the novel non-fluoroscopic three dimensional electroanatomical mapping system to monitor and analyze heart surgery in animal models

CHAPTER 6

Acute descending aortomyoplasty induces coronary blood flow augmentation

CHAPTER 7

Hemodynamic evaluation of descending aortomyoplasty versus intra-aortic balloon pump performed in normal animals: an acute study 
CHAPTER 8

Hemodynamic analysis of descending vs. ascending aortomyoplasty, and comparison with intra-aortic balloon pump

CHAPTER 9

Summary and conclusions

CHAPTER 10

Samenvatting en conclusies

Acknowledgments

Curriculum vitae

Publications 


\section{List of abbreviations}

$\begin{array}{ll}\text { IABP } & \text { Intra Aortic Balloon Pump } \\ \text { LD } & \text { Latissimus Dorsi } \\ \text { A-V shunt } & \text { Arterial-Venous Shunt } \\ \text { LVEDV } & \text { Left Ventricular End Diastolic Volume } \\ \text { LVEDD } & \text { Left Ventricular End Diastolic Diameter } \\ \text { LVESV } & \text { Left Ventricular End Systolic Volume } \\ \text { 3D } & \text { Three Dimensional } \\ \text { RV } & \text { Right Ventricle } \\ \text { LV } & \text { Left Ventricle } \\ \text { CHF } & \text { Congestive Heart Failure } \\ \text { NYHA } & \text { New York Heart Association } \\ \text { PVR } & \text { Pulmonary Vascular Resistance } \\ \text { SVR } & \text { Systemic Vascular Resistance } \\ \text { PAP } & \text { Pulmonary Artery Pressure } \\ \text { CO } & \text { Cardiac Output } \\ \text { EF } & \text { Ejection Fraction } \\ \text { CI } & \text { Cardiac Index } \\ \text { SV } & \text { Stroke Volume } \\ \text { SW } & \text { Stroke Work } \\ \text { ICU } & \text { Intensive Care Unit } \\ \text { CT } & \text { Computerized Tomography } \\ \text { MRI } & \text { Magnetic Resonance Imaging } \\ \end{array}$




\section{Introduction}

1 Heart failure 10

1.1 Chronic heart failure patients and treatment options 10

$\begin{array}{lll}1.2 & \text { Pre-clinical failure models } & 13\end{array}$

2 Cardiomyoplasty 16

2.1 Historical review 16

2.2 The surgical technique 16

2.3 Postoperative care and conditioning 17

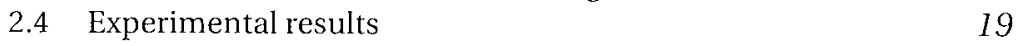

2.5 Clinical results 20

2.6 Defining the population of patients suitable for
cardiomyoplasty

$\begin{array}{lll}2.7 & \text { Summary } & 22\end{array}$

3 Aortomyoplasty 23

3.1 Background 23

3.2 The surgical technique 23

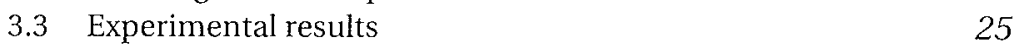

3.4 Clinical results 26

3.5 Summary 26

$4 \quad$ Monitoring and imaging modalities for experimental $\begin{array}{ll}\text { cardiac surgery } & 27\end{array}$

4.1 Swan Ganz catheter, arterial catheters, and coronary blood flow measurements $\quad 27$

4.2 Echocardiography 28

4.3 Conductance catheters 28

4.4 Electro-Anatomical mapping 28

4.5 Monitoring and imaging modalities selection for
the current studies

5 Aims of the thesis 29 


\section{HEART FAILURE}

\section{I Chronic heart failure and options for treatment}

Despite recent improvement in treatment of heart failure, this disease is still a major cause of morbidity and mortality in the Western world. Moreover, the incidence and prevalence of heart failure is increasing, accompanied by severe socio-economic implications (1). A prevalence of $1 \%$ in a population over the age of 50 was reported in the data from the Framingham study, a figure that doubles with every additional decade of age thereafter (2). The direct result of this modern epidemic is an increase in hospital admissions and an enormous annual cost $(3,4,5)$. Congestive heart failure is characterized by an impaired quality of life and markedly shortened life expectancy (6). Life expectancy of these patients depends on many variables, including the origin of the specific underlying heart disease, the age and gender of the patient, and the severity of the disease (7). According to the Framingham heart study, the one-year survival rate of patients with a new onset of congestive heart failure was $57 \%$ for men and $64 \%$ for women, and the five-year survival rate was $25 \%$ and $38 \%$, respectively (8).

Optimal medical treatment of patients with congestive heart failure improves the quality of life and survival rates, but mortality is still unacceptably high (9). The medical treatment should be based on the etiology of the heart failure (10). The two main etiologies for heart failure are ischemic heart disease and nonischemic idiopathic dilated cardiomyopathy. Ischemic heart disease is the leading cause of heart failure in Western countries (11). Digoxin treatment administered to both groups of patients demonstrated positive effects in a study that combined ischemic and nonischemic idiopathic dilated cardiomyopathy patients on both death or hospitalization (12). On the other hand angiotensin-converting enzyme (ACE) inhibitors significantly reduced mortality, mainly in the ischemic patient population (13). In addition, Carvedilol (beta-blocker), used in a program of placebo-controlled trials, was found to reduce mortality and morbidity in both ischemic and non-ischemic heart failure patients $(14,15,16)$. In a randomized trial with low dose amiodarone, a reduction in mortality and hospital admission was reported in all subgroups of severe heart failure patients (17). Finally, another medical option is intermittent treatment with intravenous inotropic agents, which showed long-term improvement in either symptoms or clinical status only in a few randomized trials $(18,19)$. Nevertheless, in the year 2000 , the annual mortality rate for medically treated heart failure patients is still above $10 \%$ (20). 
Surgical treatment is another option for end stage heart failure patients. Coronary artery bypass grafting (CABG) is considered a good choice as long as there is a viable myocardium and in the presence of graftable coronary arteries $(21,22,23)$. Although patients with low preoperative left ventricular ejection fraction (LVEF) demonstrate high short and long term mortality rates after $C A B G$, the results of the procedure are comparable to those seen in heart transplant patients at one year after surgery $(24,25)$. Asymptomatic patients usually show better results after CABG than do symptomatic patients; however, even symptomatic patients who show no post operative LVEF improvement, improve in terms of both clinical condition and survival rate after $C A B G$ to a degree similar to that demonstrated by patients with higher post operative LVEF $(26,27)$. The latest trend in CABG is toward less invasive techniques, especially off-pump surgery, which may produce even better results for high risk patients, including patients with a low $\operatorname{LVEF}(28,29)$.

To date, heart transplantation is the gold standard for end-stage heart failure treatment. It leads to a higher life expectancy and a better quality of life for this severely ill population, as compared to medically treated patients (30). On the other hand, the lack of donors and the high percentage of patients who are not suitable for heart transplantation reduce the possibility of performing this procedure on a large scale (31). The results of heart transplantation should be calculated and adjusted to reflect the $25 \%$ annual mortality of patients awaiting the operation (32). In a report from the United States, 3800 new registrations were reported in 1996, while 2300 heart transplants were performed and 740 patients died while on the waiting list (33). Moreover, chronic rejection is the main reason for the high long-term mortality rate; this factor, as well as the obstacles previously mentioned, indicate the need for other surgical options to treat patients with heart failure (34). Assessing the last registry of the International Society for Heart and Lung Transplantation is important in order to understand the current status of heart transplantation (35). The trend towards a reduction in total heart transplantation started in 1995, continued in 1998 with the lowest total heart transplant rate since 1989 (35). The majority of the transplant patients are aged 35-65, and the two dominant indications are myopathy $(45.6 \%)$ and coronary artery disease $(45.6 \%)$ (35). Overall one year survival for cardiac transplantation is $79 \%$. The patients' $50 \%$ survival is at 8.8 years and the fall off in survival is almost a straight line from year 1 through year 15 , with a constant mortality rate of $4 \%$ per year (35). The results through 1995-1998 were equal to those reported through 19911994, reaching a plateau in the improvement of results demonstrated every previous five years (35).

The introduction by Batista and coworkers of partial left ventriculectomy as an alternative for heart transplant was promising (36). Indeed, the short- 
term results were encouraging, and improvements regarding both hemodynamics and functional capacity were reported $(37,38)$. However, other reports of only $56 \%$ one-year survival without the need for heart transplantation lead to the conclusion that partial left ventriculectomy might be better indicated as a bridge to transplant (39).

Left ventricular assist devices (LVAD) were proven to be effective as a bridge to heart transplantation; moreover, the use of LVAD before heart transplant improved the post-operative results as compared to intravenous inotrope-treated patients (40). The progress in the technology of assist devices and the minimal limitations they impose on the recipient have made them a chronic treatment alternative to heart transplantation and not just a bridge to transplant $(41,42)$. However, besides the limitations to daily life and the risk of infection and thromboembolic events, the functional capacity of patients with LVAD was lower when compared to that of post heart transplantation patients (43). Another option for LVAD patients is the potential left ventricular recovery, which implies a possible weaning from the LVAD (44).

Two other surgical alternatives are the total artificial heart and heart xenografting, often referred to as the future surgical solution for end-stage heart failure $(45,46)$. However, heart xenografting, still in the stage of animal experiments, has yet to contend with problems of immunology and infection before proceeding to the clinical stage $(47,48)$. Total artificial heart was successfully implanted for long term in animals; however, in clinical work it has been used so far only as a bridge to transplant (49). Future technological and biological progress will determine whether total artificial heart will be a long-term therapy that will replace heart transplantation (50).

Dynamic cardiomyoplasty is another surgical treatment for congestive heart failure patients who are ineligible for heart transplantation (51). At present, it is clear from the immediate and long term results of dynamic cardiomyoplasty that this procedure is not suitable for the end-stage heart failure patient in New-York-Heart-Association (NYHA) Class IV, or for patients with significant mitral regurgitation or pulmonary hypertension (52). However, it is possible that dynamic aortomyoplasty, which is still in the experimental and early clinical stage of its development, may offer a surgical treatment for the end-stage heart failure patient in NYHA Class IV, or for patients with significant mitral regurgitation when cardiomyoplasty is contraindicated (53). The skeletal muscle ventricle is another technique that uses the biologic power of a skeletal muscle; however, this technique is also still in the experimental stage $(54,55)$.

This thesis is focused on cardiomyoplasty and aortomyoplasty experiments to clarify several questions regarding the two surgical techniques. The 
precise issues that will be investigated regarding cardiomyoplasty and aortomyoplasty will be discussed in sections 3 and 4 .

\subsection{Heart failure models}

During the last decade, considerable progress has been made in our understanding and management of heart failure, and several new medical and surgical therapeutic modalities have been presented (10,56). Some of these medical and surgical treatment options are currently in the preclinical stage, which explains the need for an animal heart failure model for the development and testing process. Implementation of such models provides diseased animals in which the feasibility and safety of an experimental procedure can be evaluated (57).

An ideal animal heart failure model should have a human counterpart, should closely mimic heart failure in humans and should induce reproducible heart failure at a repeatable degree of severity (58). A variety of animal heart failure models have been developed, which can be classified either according to the species and size of the animal or according to the mechanism that produces the heart failure (59). Small animal models such as rats, guinea pigs, mice, and rabbits have the advantage of being relatively inexpensive, and their short gestation period enables a large sample size to be produced within a short period of time (59). Large animal models such as dogs, sheep, swine, and goats allow a more accurate study of the left ventricular function and volumes than do rodent models, and chronic instrumentation is easier. However, higher costs and ethical problems limit their use to studies in which they are truly needed (60). The mechanisms by which heart failure is induced include rapid ventricular pacing (61), embolization or ligation of coronary arteries (62), pressure overload (63), volume overload (64), drugs (65), genetic alteration (66), viral sources (67), and idiopathic dilated cardiomyopathy (68).

Rapid ventricular pacing is used widely in large animal models and has the advantage of reproducibility and precise control of the severity of the heart failure, based on the pacing rate (69). Several weeks of chronic rapid pacing produce the syndrome of congestive heart failure, including bi-ventricular chamber dilatation, significant reduction in ejection fraction, and increase in systemic vascular resistance (70,71). Another advantage (and sometimes disadvantage) of the chronic rapid pacing model is the reversibility of the heart failure hemodynamic and neurohumoral abnormalities when pacing is stopped (72).

In several animal models, complete or partial reduction of the coronary flow is the inducing factor of heart failure. Myocardial infarction in a rat model induced by ligation of the left anterior descending coronary artery resulted in 
overt heart failure after 3-6 weeks (73). In this model, left ventricular dilatation reduced systolic function, and increased filling pressures were documented (74). If the left coronary artery is not completely ligated, heart failure may occur as a consequence of chronic myocardial ischemia (75). Coronary artery blood flow interference is widely used in large animals, as an acute model of myocardial infarction by ligation or control of constant external pressure to induce chronic stenosis in the coronary artery (76-77). Heart catheterization and intra-coronary embolization is another option for intervention in coronary blood flow (78). The results of acute infarct or chronic ischemia depend on the size of the injected polymer, the target vessel, and the number of repeated intra-coronary injections $(78,79)$. The most sophisticated model includes up to seven embolization procedures performed 1-3 weeks apart (62). Three months after the final microembolization, clinical signs of heart failure were documented, including left ventricular dilatation, decreased ejection fraction, and neurohumoral activation similar to that observed in humans with heart failure (62). This model proved that a decrease in Badrenoreceptors, L-type calcium channels, as well as sarcoplasmatic reticulum $\mathrm{Ca}^{2+}$-ATPase activity, could be induced $(80,81)$. This model has several disadvantages, including, in addition to its complexity, a high incidence of arrhythmia and mortality, that cause this model to be time consuming, technically demanding, and expensive.

Several models induce heart failure by volume overload, which results either in the creation of an arteriovenous fistula or in mitral or aortic regurgitation due to destruction of the valve. $(82,83,84)$. The size of the A-V shunt (in relation to the animal model) is the main factor for the severity of the heart dilatation and the mortality rate of the animals $(58,85)$. Flaim and coworkers reported high mortality rates $(77 \%)$ and signs of pulmonary congestion that commonly occur within 24 hours after performing the A-V shunt in small animals (86). In another animal model of inferior vena cava aortic A-V shunt, clinical signs of heart failure, such as tachycardia and a rise in LVEDP, were demonstrated (87). Pressure overload in guinea pigs, induced by descending aortic banding, caused heart failure eight weeks after the procedure (88). A canine model of gradually induced pressure overload was presented by Koide and coworkers (89). They concluded that the extent of the left ventricular muscle dysfunction could be predicted, to a large extent, according to the baseline difference between mass and wall stress that occurs naturally in healthy dogs.

Another type of heart failure can be induced by drug treatment. The drug most frequently used is doxorubicin, which results in acute and chronic cardiotoxicity and has been used in a variety of animal species (90). Siri and coworkers induced myocardial failure after 6-9 weeks with two weekly intra- 
venous injections of doxorubicin (90). Another possibility is intra-coronary administration of the drug, which allows a more precise dosage of the intra cardiac drug to be determined, and reduction of systemic intoxication (91). Many other drugs were used to induce heart failure in animal models, including epinephrine and imipramine $(92,93)$.

Several genetic heart failure models are induced by the addition or deletion of genes responsible for heart failure $(94,95)$. These models, which rely on the identification of genes that are causative for heart failure, enable the evaluation of molecular mechanisms responsible for the development and progression of the disease (96). Viral myocarditis in mice is another option for a heart failure model $(67,97)$, while several other groups use idiopathic animal heart disease as a model to study heart failure $(68,98)$. One widely used idiopathic model uses autosomal recessive cardiomyopathic strains of Syrian hamster (99). The cardiac disease proceeds progressively in several histologic and clinical phases during the life of the animal and heart failure signs appear at 7-10 months (100). Several other animal heart failure models, such as those that use more than one factor to induce heart failure or right heart failure models, are beyond the scope of this review $(101,102)$.

The purpose of the current study was to investigate the acute effect of cardiomyoplasty, i.e., the significant feature of heart failure in this model should be heart dilatation. Therefore, a carotid-jugular shunt model was chosen to induce volume overload. Several factors need to be tested before the implications of this model can be evaluated in relation to the study of heart failure: the acute effect of the shunt, the reversibility of the heart dilatation, as well as measurement of the chronic heart dilatation generated after creation of the shunt. Once these factors have been tested and documented it is possible to decide on the correct timing of the cardiomyoplasty procedure.

In summary, there are numerous animal heart failure models; however, none of them is an ideal model for studying the physiology and treatment of heart failure in man. The question of which animal model to use should be based on the understanding of each model's advantages and disadvantages, as well as the investigator's own experience. We chose the goat carotid-jugular A-V shunt model to investigate the acute effects of cardiomyoplasty. However, more data regarding the model should be gathered before the implications of cardiomyoplasty can be investigated. 


\section{CARDIOMYOPLASTY}

\section{I Historical review}

The concept to use skeletal muscle as a source of energy to augment failing hearts has a long history. In the past, skeletal muscle was used passively for the structural repair of the heart. In a first report from 1931, De Jesus described the use of the pectoralis muscle to repair a traumatic laceration of the left ventricle (103). In 1935, Beck described the use of a pectoralis flap, while preserving its neurovascular pedicle around an ischemic ventricle to treat patients with angina pectoris (104). As early as 1948, Petrovsky began using the diaphragmatic muscle to reinforce aneurysms of the heart in more than 100 patients (105).

The first attempt to assist the heart with an electrically stimulated skeletal muscle was published in 1959 (106), and the latissimus dorsi was first used in 1966. When electrically stimulated, a blood pressure of 60 to $80 \mathrm{mmHg}$ was obtained during periods of ventricular fibrillation (107). The main problem of these trials was the inevitable occurrence of skeletal muscle fatigue after some minutes of continuous stimulation. The breakthrough was achieved with the report of Salmons and co-workers, which described the possibility of converting a skeletal muscle into a fatigue-resistant muscle by continuous administration of slow electrical stimulation $(108,109)$. The second major step was the development of a special stimulator able to produce a burst of electrical pulses that mimics the long titanic contraction of the myocardium. This stimulator is also capable of sensing spontaneous heart beats, thus enabling synchronization of contractions (110). The first clinical application of these advances was performed by Carpentier and co-workers in 1985 (111). A left latissimus dorsi flap was used to replace part of the ventricle, after resection of a large cardiac tumor. Later, the same group and others described dynamic cardiomyoplasty in other patients, as a surgical procedure providing a power source to enhance failing hearts (112-113). In the fifteen years after the first clinical success, more than 1000 patients worldwide underwent this operation, and a considerable amount of research has been performed.

\subsection{The surgical technique}

The surgical technique of dynamic cardiomyoplasty has been described extensively by Chachques and co-workers (112). Under general anesthesia, the patient is initially placed in the right lateral position. The left latissimus dorsi muscle is exposed through a flank incision extending from the axilla to the mid point of the 12 th rib. The muscle is freed from the surrounding tissue 
while ligating and cutting the collateral blood vessels that supply mainly its distal part. The proximal neurovascular pedicle is preserved. Two stimulating electrodes are connected to the muscle, the proximal, negative electrode is connected in close proximity to the course of the main nerve branches, and the distal, positive electrode is implanted transversely into the muscle $4-6 \mathrm{~cm}$ distally. At this stage, measurements of the muscle stimulation threshold and muscle recruitment are obtained using an external stimulator. A $6 \mathrm{~cm}$ segment of the anterior portion of the second rib is then resected to allow transposition of the latissimus dorsi muscle flap (LDMF) into the thorax. The muscle is inserted into the pleural cavity, and its tendon is cut and sutured to the periosteum of the third rib, while closing the thoracic window. The cutaneous flaps are then sutured to the thoracic wall with interrupted absorbable sutures, and the wound is closed after positioning of the subcutaneous drainage.

In the second stage of the operation, the patient is placed in the supine position and the heart is exposed through a median stemotomy incision. To introduce the LDMF and the pacing leads, a long incision is made in the left pleura. Two epicardial sensing electrodes are connected to the right ventricle, and sensing and impedance measurements are performed. The heart is wrapped with the LDMF and the flap is secured with stitches to the myocardium or pericardium. Should extreme heart dilatation not allow complete muscle wrapping, a piece of autologous pericardium can be used to complete the wrapping. A pocket for the cardiomyostimulator is then fashioned beneath the left rectus abdominis muscle, and after connection of all leads to the stimulator, the sternotomy is closed.

Cardiopulmonary bypass is generally not required because surgery is performed on the beating heart, unless concomitant cardiac surgery is carried out. Nevertheless, it should always be available on standby.

\subsection{Postoperative care and conditioning}

Due to the poor cardiovascular performance of patients after cardiomyoplasty, the reported mean duration of stay in an intensive care unit is 8.8 days, the mean period of assisted ventilation is 4.5 days, and the mean total postoperative hospital stay is as long as 36 days (114). After allowing two weeks of delay for flap recovery (vascular delay) and for the formation of adhesions between the LDMF and the myocardium, the conditioning protocol is started $(114,115)$.

Conditioning is necessary because there are major differences between skeletal and cardiac muscles. The specific morphologic structure of cardiac muscle cells enables them to contract in an all-or-none fashion (functional 
syncytium), remain contracted for a longer period, and work continuously without fatigue (116). Cardiac muscle cells are equipped with an abundance of mitochondria, thus producing enough ATP for continuous work without incurring oxygen debt. In contrast, skeletal muscle cells have a different morphologic structure: they are organized in motor units, remain contracted for a shorter period, and develop an oxygen debt and fatigue after some minutes of continuous work. In addition, there are several types of skeletal muscle cells. The two main skeletal muscle fibers are Type I and Type II. Type I is characterized by slow twitch contraction, containing a large number of mitochondria, thus enabling aerobic work and having relative fatigue resistance. This is in contrast with the fast twitch Type II fibers, which have a small number of mitochondria and are thus restricted to anaerobic work and display fatigue after a few minutes of continuous work. The relative number of these two main skeletal fiber types determines the muscle's functional qualities. Type II muscle fibers constitute from 50\% (117) upto 60\% (118) of the human latissimus dorsi. Therefore, the human latissimus dorsi tends to fatigue after some minutes of continuous work. Following the reports of Salmons and co-workers, conditioning protocols have been developed to transform fast twitch Type II muscle fibers into slow twitch Type I muscle fibers. After twelve weeks of conditioning, the majority of the muscle fibers become slow twitch Type I, and the muscle is relatively fatigue resistant (109).

In experimental animals, an undesirable effect of muscle transformation was the striking reduction in contractility and power (119). Another secondary sequela was late chronic damage to the LD muscle (120). To prevent such chronic muscle damage, adaptations were made to the previous stimulation protocol, reducing the frequency of LD muscle contractions. The most recent modification was to stimulate the muscle at a low ratio of $1: 6$ while the patient's heart rate is low (usually during sleep). During higher heart rates, the duration of the delivered burst of pulses is shortened and the muscle stimulation ratio is increased.

Davidse and coworkers demonstrated that transformation to Type I fibers occurs in up to $80 \%$ of cardiomyoplasty patients, and this is despite reports that Type I fibers were found to be reduced in heart failure patients $(121,122)$.

The conditioning protocol takes twelve weeks, during which the number of the electrical pulses in a burst is gradually increased every two weeks. The final stimulator setting is usually six pulses in a burst duration of 185 milliseconds, every second spontaneous beat when a high heart rate is sensed, and every sixth spontaneous beat when a slow heart rate is sensed. The exact parameters and voltage of the pulse are adjusted individually for each patient. 


\subsection{Experimental results}

Most attempts to demonstrate systolic augmentation in healthy animal hearts that have undergone cardiomyoplasty have failed (123). According to the hypothesis that systolic augmentation can be significant only when the procedure is applied to a failing heart, animal experiments have focused on models of heart failure. Millner and co-workers demonstrated a significant improvement in cardiac output and stroke volume with a significant reduction of pulmonary artery capillary wedge pressure and left ventricular end-diastolic pressure, while pacing the latissimus dorsi flap (124). These results were demonstrated in sheep with heart failure induced by ligation of a coronary artery. In another model, heart failure was induced in dogs by intravenous treatment with Adriamycin (Table 1) (125). In this model, a considerable improvement of right and left heart performance was demonstrated after cardiomyoplasty.

Due to the difficulties of demonstrating such significant hemodynamic improvement in humans, an effort was made to explore another physiological explanation for clinical improvement in patients after cardiomyoplasty. Lee and co-workers demonstrated a significant reduction in systolic wall stress after cardiomyoplasty, and suggested that it is this reduction, followed by reduction of myocardium oxygen consumption, that is responsible for the patient's well-being (126). Mannion and co-workers published several reports about the presence of collateral blood vessels from the latissimus dorsi muscle flap to the myocardium (127). In a later work of this group, a rise in collateral blood flow was achieved after chronic electrical stimulation (128).

Another hypothesis is the girdling effect of the latissimus dorsi muscle flap on the geometric structure of the heart. This girdling effect has a reverse remodeling influence on the heart, and possibly prevents the ongoing development of dilatation in the end stage of congestive heart failure. Capouya and co-workers demonstrated prevention of further dilatation and improvement in ejection fraction after cardiomyoplasty, even without electrical stimulation (129). A significant reduction in the size of the dilated goat heart was demonstrated in our laboratory three months after dynamic cardiomyoplasty (130). These findings were observed three months after performing a carotid artery jugular vein shunt to induce cardiac dilatation. 
Table 1. Hemodynamic data of cardiomyoplasty on biventricular function in a canine model with adriamycin induced chronic heart failure, according to Cheng et al., 1993 (125)

$\begin{array}{llll}\text { Variable } & \text { Without assistance } & \text { With assistance } & \text { P. Value } \\ \text { Left diastolic pressure }(\mathrm{mm} \mathrm{Hg}) & 11.0 \pm 1.6 & 9.6 \pm 1.5 & \mathrm{p}<0.05 \\ \text { Right systolic pressure }(\mathrm{mm} \mathrm{Hg}) & 21 \pm 2 & 26 \pm 3 & \mathrm{p}<0.05\end{array}$

\subsection{Clinical results}

Throughout the last decade, data have been published that summarize the follow-up of patients up to seven years after the operation $(115,131,132)$.

Mortality. In the early years of the clinical procedures, the mortality rate was rather high (115). Carpentier and co-workers reported a 54\% perioperative mortality rate in patients who underwent cardiomyoplasty between 1985-1987, compared to a perioperative mortality rate of $12.8 \%$ in patients who underwent the same procedure between 1988 and 1992. This striking change can most likely be ascribed to the development of the surgical technique, experience, and the improvement in patient selection. In a five-year follow-up of this group of patients, the late mortality rate was $30 \%$. According to the report of Carpentier, this rate is still less than the mortality rate in equivalent groups of patients who received only medicinal treatment. Moreira and co-workers published the results of a five-year follow-up of 31 patients after cardiomyoplasty (132). There was no perioperative mortality. The late mortality was significantly influenced by the functional state of the patients before the operation. The five-year mortality rate was $40 \%$ in the group of patients in functional class III according to the New York Heart Association (NYHA). In contrast, there was a $100 \%$ mortality rate in the group of patients in functional class IV. Other groups in which cardiomyoplasty was performed more recently had low perioperative mortality rates. The group from Lyon, France, reported $0 \%$ mortality six months after the operation (114). Analysis of the causes of the late mortality showed that more than $50 \%$ was caused by sudden cardiac death, most probably due to a malignant ventricular arrhythmia (114). These results encourage the current development of a combined myostimulator and defibrillator.

Functional capacity. All groups reported a significant improvement in functional capacity after surgery: a mean functional class of 3.2 NYHA before the operation, compared to a mean 1.7 NYHA after the cardiomyoplasty (132). In addition, a significant improvement in exercise capacity, a considerable reduction in drug use, and a significant reduction in the frequency of hospitalization due to congestive heart failure were reported (Table 2) (115). 
Table 2. Functional improvement in 29 patients surviving cardiomyoplasty and not undergoing heart transplantation, according to Carpentier et al, 1993 (115)

$\begin{array}{llll}\text { Variable } & \text { Preop. } & \text { Postop. } & \text { P. Value } \\ \text { NYHA Functional class } & 3.3 & 1.6 & \mathrm{p}<0.05 \\ \text { Exercise capacity }(\mathrm{W}) & 60 \pm 24 & 96 \pm 18 & \mathrm{p}<0.1 \\ \text { Hospitalizations per patient per year (no.) } & 2.4 & 0.4 & \mathrm{p}<0.05 \\ \text { Oxygen consumption }(\mathrm{ml} / \mathrm{min} / \mathrm{kg}) & 12.8 \pm 3.5 & 18.6 \pm 4 & \mathrm{p}<0.05\end{array}$

Hemodynamics. Hemodynamic improvements were reported as many as three years after the operation, but usually these changes were small and did not always correspond to patients' clinical condition. In the group of patients operated by Carpentier and co-workers, ejection fraction was significantly higher two years after cardiomyoplasty, at $30 \%$ compared to $24 \%$ at baseline (115). In addition, a significantly higher cardiac index was recorded. None of the other hemodynamic parameters demonstrated statistically significant changes. Moreira and co-workers noticed a significant improvement in the ejection fraction after cardiomyoplasty, but also a slow retum to baseline values five years after the operation (52).

Due to the moderate hemodynamic improvement in contrast to the major clinical improvement as well as the experimental knowledge gained regarding the physiological explanation for this clinical improvement, recent efforts have focused on demonstrating these changes in patients. Bellotti and coworkers reported a significant reduction in wall stress of the left ventricle in patients one year after surgery, while the stimulator was turned on (133). Lately, Kass and co-workers reported a reduction in end-systolic volume in patients one year after the operation (134). The authors offer the hypothesis that clinical improvement can be ascribed to the reduction of the ventricular size and partially to the reversal of chamber remodeling due to heart failure. These results were demonstrated by pressure volume loops attained using the conductance catheter. In addition, the author suggests that NYHA class-IV patients had a higher mortality rate due to the greater myocyte loss replaced by fibrosis and that their heart may be less capable of reverse chamber remodeling. Using the same conductance catheter technology, Schreuder and coworkers demonstrated hemodynamic improvement in patients after cardiomyoplasty while establishing the exact timing of the electrical burst based on data gathered in real time from the pressure volume loops (135). One should be aware that, today, a wide randomized cardiomyoplasty trial is being 
carried out in the USA, but the results are expected to be published only by the end of this year.

\subsection{Defining the population of patients suitable for cardiomyoplasty}

This section summarizes the preclinical and clinical insights gathered to date, in order to define the patients for whom the procedure is suitable. The selection of suitable patients, who can benefit from cardiomyoplasty, is important because significant reduction in the perioperative mortality is related to better selection of patients (115). In 1992, Furnay summarized the preoperative risk factors for cardiomyoplasty perioperative mortality (136). Suitable patients should demonstrate symptoms of NYHA functional class III, i.e., congestive heart failure symptoms after minor effort, but not at rest. The mortality rate was high in patients who demonstrated preoperative NYHA functional class IV, i.e., congestive heart failure symptoms at rest. In addition, the author recommended excluding patients with right heart failure and pulmonary hypertension.

Results from centers in France and Brazil indicate that it is possible to characterize patients suitable for cardiomyoplasty $(115,132)$ Patients should be under the age of 70, demonstrate symptoms of NYHA functional capacity III, congestive heart failure preferably in sinus rhythm. Significant ventricular arrhythmia, NYHA functional class IV, severe increased left ventricular enddiastolic volume $(>300 \mathrm{ml})$, and poor left ventricular function (ejection fraction $<10 \%$ ) are contraindications to the procedure.

These precise criteria limit the number of patients for whom the operation is suitable. Moreover, since most cardiologists prefer to treat patients in NYHA functional capacity class III conservatively rather than resorting to a major operation, the number of patients is even further limited. The existence of other, less invasive options, the absence of encouraging clinical results before more data had been gathered from the recent important preclinical studies, as well as economical considerations caused the procedure to be abandoned by most centers.

\subsection{Summary}

Cardiomyoplasty is a surgical treatment for a well-defined group of patients who suffer from congestive heart failure. The procedure is not a contraindication to future heart transplantation, when indicated. The clinical results up to seven years after cardiomyoplasty are encouraging, but there is still a gap between significant functional capacity improvement of the patients after cardiomyoplasty and the moderate hemodynamic change. Experimental data 
and recent measurements in patients provide new insights in understanding the physiologic effect of cardiomyoplasty. Nevertheless, since the procedure did not gain wide popularity and is most likely not suitable for NYHA functional capacity class IV patients, aortomyoplasty may be another skeletal muscle application that should be investigated as a potential treatment for stage IV heart failure patients.

\section{AORTOMYOPLASTY}

\subsection{Background}

The intra-aortic balloon pump (IABP), proven to be a useful clinical tool for support of the heart failure patient, was found to be especially effective in the setting of acute ischemia. The IABP causes decreases in peak systolic pressure and myocardial oxygen consumption, and increases in mean diastolic blood pressure and coronary blood flow $(137,138,139)$. However, long-term support by the IABP is not possible, due to the occurrence of infection and thromboembolic events (140). This has raised new interest in the concept of diastolic augmentation using a conditioned skeletal muscle as a power source for external aortic counterpulsation. Since 1990, considerable experimental and preliminary clinical data on dynamic aortomyoplasty have been gathered $(141,142)$.

\subsection{The surgical technique}

There are two different methods of performing dynamic aortomyoplasty. Firstly, by wrapping the latissimus dorsi muscle around the ascending aorta using the right latissimus dorsi muscle (141), and secondly by wrapping the descending aorta using the left latissimus dorsi muscle (142). The first part of the operation, the preparation of the latissimus dorsi muscle, is identical to the first part of the cardiomyoplasty procedure, and has been described in detail by Chachques and co-workers (112). Under general anesthesia, the patient is initially placed in the left or right lateral position according to the side of the latissimus dorsi that will be used. The latissimus dorsi muscle is exposed through a flank incision extending from the axilla to the mid point of the 12 th rib. The muscle is freed from the surrounding tissue while ligating and cutting the collateral blood vessels that supply mainly its distal part. The proximal neurovascular pedicle is preserved. Two stimulating electrodes are implanted in the muscle, the proximal, negative electrode is connected in close proximity to the course of the main nerve branches, and the distal, posi- 
tive electrode is implanted transversely into the muscle 4-6 cm distally. At this stage, the muscle stimulation threshold and muscle recruitment are measured using an external stimulator.

The second stage of the operation, the wrapping of the aorta, varies according to the aortic portion intended for wrapping. Chachques and coworkers originally proposed the ascending aorta for aortomyoplasty, based on the major benefits of performing aortic counterpulsation as close as possible to the aortic valve (141). In this case, a right LDM is used because of preferred anatomical orientation and proximity. Aortic root enlargement is performed using a pericardial patch, to increase the amount of blood displaced by the LDM contraction during the aortic counterpulsation, as described in animal studies. When the ascending aorta is relatively short, the muscle can be split and wrapped proximally and distally to the innominate artery around the aorta (141).

Wrapping of the descending thoracic aorta has been proposed as an alternative method of aortomyoplasty (142). Such a technique may present some advantages when compared to the ascending aortomyoplasty, since the portion of the aorta suitable for the skeletal muscle wrapping is longer. In case of re-operation due to previous cardiac surgery, resternectomy and mediastinal dissection are avoided in this approach. Muscle preparation and the wrapping of the aorta can be achieved through a single skin incision, which is advantageous because it is simple, short, and a less traumatic procedure than the ascending aortomyoplasty (143). Nevertheless, descending aortomyoplasty usually requires the ligation of one or more intercostal arteries with the subsequent potential complication of postoperative neurological disorders (Adamkiewitz artery). Cernaianu recently reported a modification of the descending aortomyoplasty operation, which attempts to preserve the intercostal arteries (144). Indeed, studies have demonstrated the use of muscle splitting at the level of the intercostal artery and of pericardial "bridges" to connect the LDM posteriorly to the wrapped aortic portion. Cardiopulmonary bypass is not generally required, unless concomitant cardiac surgery is carried out. Nevertheless, it should always be available on standby. The aortomyoplasty surgical procedure is still under extensive experimental and clinical evaluation. Improvement and refinement of the surgical technique is still likely, and may lead to wider clinical applications in the future.

The conditioning protocol for transforming the skeletal muscle fibers into Type I, slow twitch, fatigue resistant fibers is the same as after the cardiomyoplasty procedure and is discussed in section 2.3 of the introduction. There are not sufficient clinical data at this moment to discuss the postoperative care of the aortomyoplasty patients. 


\subsection{Experimental results}

Both ascending and descending aortomyoplasty reveal significant hemodynamic improvements when the latissimus dorsi muscle (LDM) wrapped around the aorta is stimulated. Chachques and co-workers demonstrated that stimulation of the LDM wrapped around the ascending aorta resulted in significant diastolic augmentation in goats, up to a diastolic pressure of 90 $\mathrm{mmHg}$ (compared to a baseline of $115 / 65 \mathrm{mmHg}$ ) (141). The results were even better when using a pericardial patch to enlarge the ascending aortic cavity: diastolic pressures reached $105 \mathrm{mmHg}$ (baseline $105 / 70 \mathrm{mmHg}$ ). With the aortic patch there was a significant increase in the subendocardial viability index (diastolic pressure-time index/systolic tension-time index), from $29 \%$ to $42 \%$, using a high-dose of propranolol to reduce cardiac contractility. The same group presented the experimental results 12 and 24 months postoperatively with a conditioned LDM (145). Two years after the operation, diastolic augmentation could still be observed. While inducing cardiac failure by propranolol, stimulation of the LD muscle resulted in a significant decrease in left ventricular end-diastolic pressure and systemic vascular resistance, and a significant increase in cardiac output, from $3.6 \pm 0.5$ to $5.5 \pm 1.0 \mathrm{l} / \mathrm{min}$. The examination of histological structure two years after the wrapping procedure showed preservation of the aortic wall structure and tight adhesions between the aortic adventitia and the LDM. (145).

Dynamic ascending aortomyoplasty also revealed clear hemodynamic benefits in an animal study carried out by Lazzara and co-workers. They demonstrated a significant increase in mean diastolic blood pressure, from 62 $\pm 4 \mathrm{mmHg}$ to $71 \pm 3 \mathrm{mmHg}(\mathrm{p}<0.05)$ and EVR (index of myocardial diastolic perfusion and ventricular oxygen demand) (146). During this study in dogs, heart failure was induced by rapid ventricular pacing, and the procedure was monitored using the conductance catheter technique. The pressure volume loops demonstrated a shift to the left, which indicates a decrease in both left ventricular end-diastolic and end-systolic volumes, as well as the reduction of systolic pressures during skeletal muscle contraction (146).

These are indeed encouraging pre-clinical results; however, some important aspects are still missing in the pre-clinical study. First, two major hemodynamic effects of IABP have not yet been proven to occur when the skeletal muscle is contracted around the aorta: augmentation of coronary flow and afterload reduction. Several authors have reported diastolic pressure augmentation $(141,144)$, and although one may assume that this will lead to coronary blood flow augmentation, this assumption was not tested in the aortomyoplasty experiments. The factor of afterload reduction, which is induced by the IABP, has been overlooked by most authors. However, it is an 
important hemodynamic effect, especially for heart failure patients, and should be evaluated at the pre-clinical phase. The other important issue currently missing in the pre-clinical stage is an objective comparison between the two main surgical techniques, ascending and descending aortomyoplasty. If one of the surgical techniques turns out to be better, it should be used at the clinical stage; if different advantages are proven in each technique, this finding may help the surgeon to choose the optimal surgical option according to the patient's individual needs.

\subsection{Clinical results}

The clinical experience of dynamic aortomyoplasty is very limited. Since November 1992, 14 clinical aortomyoplasty procedures have been performed worldwide in four different centers (147). The mean age of these patients was $56 \pm 7$ years, 10 men and four women, of whom nine had ischemic cardiomyopathy and five had idiopathic dilated cardiomyopathy. The mean end-diastolic left ventricular diameter was $82 \pm 6 \mathrm{~mm}$, the mean preoperative New York Heart Association (NYHA) functional class was 3.4, and the mean left ventricular ejection fraction was $16 \% \pm 4 \%$. The aortomyoplasty technique was either ascending aorta wrapping $(n=11)$ or descending aorta wrapping $(n=3)$. Cardiopulmonary bypass was not used in any of these cases. Hospital mortality was $14 \%$. During a mean follow-up of 9 months, there was an additional $36 \%$ death rate. Postoperatively, there was a significant improvement in NYHA functional class and in patients' quality of life. Diastolic augmentation was demonstrated by hemodynamic and Doppler echocardiographic studies. In another report of clinical experience, by Mesana and coworkers, two of the four patients died at the immediate postoperative stage and only one patient survived one year (148). At one year, clear hemodynamic and clinical improvements were documented. However, it is important to note that this procedure is still in the early stages of clinical evaluation and the number of patients treated is too small to show any statistical significance. Furthermore, current studies do not indicate which patient is suitable for dynamic aortomyoplasty. Finally, the data available are, as yet, insufficient to address the question regarding preference for one technique over the other when comparing ascending and descending aortomyoplasty.

\subsection{Summary}

Aortomyoplasty is a surgical procedure which should sustain the hemodynamic effects of the intra-aortic balloon pump in the long-term. This operation will probably be suitable for end-stage heart failure patients. The experi- 
mental results are encouraging, but further experimental studies are needed, especially to measure coronary blood flow augmentation, assess afterload reduction, and conduct a comparative analysis of ascending and descending aortomyoplasty.

\section{MONITORING AND IMAGING MODALITIES FOR EXPERIMENTAL CARDIAC SURGERY}

The importance of imaging and monitoring techniques for the evaluation of cardiac surgery research cannot be overestimated. However, an objective comparison between different techniques is hard to find in the literature, and neither are there recommendations regarding preference of a monitoring technique or the analysis of a particular procedure in cardiac surgery. Some of the monitoring techniques and imaging modalities require considerable training and experience, and it can therefore be anticipated that most investigators will limit their evaluation, depending on knowledge and experience The objective of this chapter is to provide an overview of imaging and monitoring modalities used in this thesis and to compare them, in order to explain why a particular modality was selected for each study.

\subsection{Swan Ganz, arterial catheters, and coronary blood flow measurements}

The clinical and experimental importance of the pulmonary arterial catheter is well established since its first introduction by Swan and coworkers (149). Indeed, numerous experimental and clinical works were based on this technique, which is known as a reliable method for calculating cardiac output, systemic and pulmonary resistance, and wedge pressure, representing left-ventricular end-diastolic pressure $(150,151)$. However, it is important to note that this technique is not errorless and that results should be interpreted with caution $(152,153)$. The Swan-Ganz catheter measures several heartbeats together, is incapable of sensing the difference between succeeding beats, and is unable to generate ventricular volumes and additional details concerning the left ventricular regional hemodynamics. A simple and basic technique is arterial catheters, which are widely used clinically and experimentally; however, the poor correlation between the arterial blood pressure and the actual hemodynamic status has been well documented (154). Several authors suggested extrapolation of the arterial pressure curve contour to generate cardiac output measurements (155); however, while for clinical purposes this noninvasive technique may be advantageous, its advantage is less relevant in experimental work, and until now the accuracy of the technique has not been sufficient $(156,157)$. 
Coronary blood flow measurements can be attained using several methods; for our aortomyoplasty studies we chose to use the Doppler flow probe technique, which was proven to be accurate enough to record relative velocity changes (158).

\subsection{Echocardiography}

Echocardiography is a well-established tool for clinical evaluation (159). However, the use of echocardiography as an objective tool is under debate, especially for pre-clinical research (160). Echocardiography is capable of generating three-dimensional geometric information of the heart. Improvements in the $3 \mathrm{D}$ as compared to $2 \mathrm{D}$ echocardiography are important in this aspect $(161,162)$. However, although several methods were developed to facilitate automatic border detection, the main drawback of echocardiography for experimental use is the subjective bias of the operator and analyzer of the exam $(163,164,165)$. Even regarding the accuracy of the left ventricular volumes, echocardiography was found to be accurate only when the left ventricle's volumes were at steady state, and this is insufficient for an experimental study of the operation at more extreme preload situations $(166,167)$. While non-invasiveness and speed of data acquired are clinical advantages of the echocardiography technique, they are clearly less important at the preclinical, experimental stage (168).

\subsection{Conductance catheters}

The conductance catheter technique is clinically and experimentally well established, and was proven to be highly accurate when compared to other modalities (169). The system is capable of generating beat-to-beat real time pressure volume loops and is highly sensitive to minor hemodynamic differences between the beats (170). This is an important feature for the understanding and analysis of cardiac interventions, such as IABP, certain assist devices, cardiac pacing, and operations such as aortomyoplasty and cardiomyoplasty, which do not assist every spontaneous beat. The main drawback of the conductance catheter technology as compared to echocardiography and electroanatomical mapping is its inability to evaluate hemodynamic changes in different regions within the left ventricle.

\subsection{Electroanatomical mapping technique}

The novel three-dimensional electroanatomical mapping system was proven to be highly accurate and reproducible both in-vitro and in-vivo $(171,172)$, 
and is currently clinically used for several cardiologic imaging and intervention procedures, such as hemodynamic evaluation, distinguishing infarcted from healthy myocardium, ablations, and direct myocardial revascularization $(172,173,174,175,176)$. As mentioned earlier, one of the advantages of the electroanatomic mapping technique over the conductance catheter technique is its ability to demonstrate regional changes within the left ventricle (177).

The ability of the system to be tagged and to reconstruct the 3D beating left ventricle enabled a beat-to-beat analysis of the mean map gathered over 20-30 minutes of the beating heart. This makes the system less sensitive for differences between beats than the conductance catheter technique and more vulnerable to animal or patient instability. The ability of the system to sense and present the endocardial activation propagation may be important for the analysis of surgery that interferes with endocardial integrity, such as the Batista operation and aneurysmectomy procedures, as well as the Mase procedure and its modifications.

\subsection{Monitoring and imaging modalities selection for the current studies}

The decision as to which monitoring and imaging techniques are to be used in the current studies was determined according to the objective of each study. For the cardiomyoplasty experiment, previous data suggest that the conductance catheter technique is highly sensitive and the ideal technique to distinguish differences between assisted and unassisted beats $(178,179)$. SwanGanz was used mainly for the calibration of the conductance catheter measurements. However, when more 3D information was needed about the exact geometrical changes of the assisted systole, the electroanatomical technique was the imaging technique of choice. Echocardiography was used to gain more information about left ventricular dilatation induced by the $A-V$ shunt and to determine the correct timing for the cardiomyoplasty procedure. For the purpose of the aortomyoplasty studies, solid-state arterial pressure and coronary blood flow measurement techniques were sufficient to demonstrate whether the operation induced afterload reduction and coronary blood flow augmentation comparable to those induced by the IABP.

\section{AIMS OF THE THESIS}

The goal of this work is to offer a contribution to the field of surgical treatment for heart failure patients. The search for new insights in the understanding of cardiomyoplasty and aortomyoplasty brings to the forefront debates regar- 
ding the procedures per se as well as the significance of new clinical data. for treatment of end stage heart failure patients.

For this purpose, this thesis will focus on and clarify several hemodynamic issues concerning the animal pre clinical stage of both cardiomyoplasty and aortomyoplasty.

In the cardiomyoplasty studies the acute peri-operative and immediate postoperative stages were examined, and more specifically, the hemodynamic and geometrical changes to the left ventricle that resulted from the wrapping and from the stimulation protocol of the latissimus dorsi muscle were studied. In the aortomyoplasty studies, the procedure to induce coronary blood flow augmentation and afterload reduction were studied and compared to the findings with those induced by the IABP. A second goal of the aortomyoplasty studies was to perform a hemodynamic comparison between two techniques using the ascending or descending wrap.

For the cardiomyoplasty studies, an animal dilatation model was chosen to mimic the clinical condition of end stage congestive heart failure patients. The need to gather more data regarding this animal model before proceeding to the cardiomyoplasty study is addressed in Chapter 2.

In Chapter 3, using a conductance catheter in a goat model of carotidjugular A-V shunt, the peri-operative hemodynamic and geometrical changes that result from the wrapping procedure were tested. In Chapter 4 , the same model was used to test the effect of the stimulation protocol of the latissimus dorsi muscle.In Chapter 5 the novel electroanatomical mapping technique was used to generate new data concerning the exact squeezing mechanism of the skeletal muscle around the left ventricle. The aim of Chapter 6 was to test whether aortomyoplasty actually induces coronary blood flow augmentation, while in Chapter 7 the two major hemodynamic effects of the IABP, coronary blood flow augmentation and afterload reduction, were compared between descending aortomyoplasty and the IABP.

The study presented in Chapter 8 was designed as an objective comparison between the two main aortomyoplasty techniques and the gold standard, the IABP, in order to specify the hemodynamic benefit of each.

For these seven pre-clinical studies, several important cardiac imaging and monitoring technologies were used. Our experience with all these modalities, a comparison of the imaging and monitoring techniques, as well as guidelines for choosing the best monitoring and imaging modality according to the aim of the study will be summarized and included in Chapter 9. Also presented in this chapter are the conclusions drawn from each of the previous chapters. 


\section{REFERENCES}

1 Armstrong PW, Moe GW. Medical advances in the treatment of congestive heart failure. Circulation 1994;88:2941-2952.

2 Kannel WB, Belanger AJ, Epidemiology in heart failure. Am Heart J. 1991;121:951-957.

3 Reitsma JB, Mosterd A, De Cream AJM, Koster RW, Van Capello IJL, Grobbee DE, Thyssen JGP, Increase in hospital admission rates for heart failure in 'The Netherlands, 1980-1993. Heart 1996;76:388-392.

4 Yancy CW Firth BG. Congestive Heart Failure. Dis. Mon. 1988;34:467-536.

5 Garg R, Packer M, Pitt B, Yusuf S. Heart failure in the 1990s: evolution of a major public

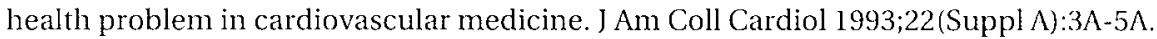

6 Cohn JN. Current therapy of failing heart. Circulation 1988; 78:1099-1107.

7 Parameshwar J, Keegan J, Sparrow J, Sutton GC, Poole-Wilson PA. Predictors of prognosis in severe chronic heart failure. Am Heart J 1992; 123: 421-426.

8 Ho KL, Anderson KM, Kannel WB et al. Survival after the onset of congestive heart failure in Framingham Heart Study subjects. Circulation 1993; 88:107-115.

9 Swedberg K. Reduction in mortality by pharmacological therapy in congestive heart failure. Circulation 1993; 87 (suppl. IV): IV-126-IV-129.

10 Follath F, Cleland JGF, Klein W, Murphy R. Etiology and response to drug treatment in heart failure. J Am Coll Cardiol 1998;32:1167-1172.

11 Codd MB, Surge DD, Gersh BJ, Melton LJ. Epidemiology of idiopathic dilated and hypertrophic cardiomyopathy. A population-based study in Olmsted County, Minnesota, 1975-1984. Circulation 1989;80:564-572.

12 Digitalis Investigators Group. The effect of digoxin on mortality and morbidity in patients with heart failure. N Engl J Med 1997;336:525-533.

13 Nony P, Boissel JP, Girard P, Leizorovicz A, Lievre M, Chifflet R. Relative efficacy of angiotensin converting enzyme inhibitors on mortality of patients with congestive heart failure: implications of randomized trials and role of the etiology (ischemic or non-ischemic) of heart failure. Eur Heart J 1992;13:1101-1108.

14 Packer M, Bristow MR, Cohn JN, et al. for the US Carvedilol Heart Failure Study Group. The effect of carvedilol on morbidity and mortality in patients with chronic heart failure. N Engl J Med 1996;334:1349-1355.

15 Bristow MR, Gilbert EM, Abraham WT, et al., for the MOCHA Investigators. Carvedilol produces dose-related improvements in left ventricular function and survival in patients with chronic heart failure. Circulation 1996;94:2807-2816.

16 Packer M, Collucci WS, Sackner-Bernstein JD, et al., for the PRECISE Study Group. Double-blind, placebo-controlled study of the effects of carvedilol in patients with moderate to severe heart failure. The Precise Trial. Circulation 1996;94:2793-2799.

17 Doval HC, Hul DR, Grancelli HO, Perrone SV, Bortman GR, Curiel R. Randomized trial of low-dose amiodarone in severe heart failure. Lancet 1994;344:493-498.

18 Colucci WS, Elkayam U, Horton PD, et al. Intravenous nesiritide, a natriuretic peptide, in the treatment of decompensated congestive heart failure. N Engl J Med 2000;343: 246-253.

19 Elis A, Bental T, Kimchi O, Ravid M, Lishner M. Intermittent dodutamine treatment in patients with chronic refractory congestive heart failure: a randomized double-blind placebo-controlled study. Clin Pharmacol Ther 1998;63:682-685.

20 Pitt B, Poole-Wilson PA, Segal R, Martinez FA, Dickstein K, Camm AJ, Konstam MA, Riegger G, Klinger GH, Neaton J, Sharma D, Thiyagarajan B. Effect of losartan compared 
with captopril on mortality in patients with symptomatic heart failure: randomized trial-The Losartan I Ieart Failure Survival Study ELITE II. Lancet 2000;355(9215): 1582-1587.

21 Letsou GV. Selection of appropriate patients with heart failure for aortocoronary bypass surgery. Curr Opin Cardiol 1999;14(3):230-233.

22 Pagano D, Lewis ML, Townend JN, Davies P, Camici PG, Bonser RS. Coronary revascularisation for postischemic heart failure: how myocardial viability affects survival. Heart 1999;82(6):684-688.

23 Mickleborough LL, Carson S, Tamariz M, Ivanov J. Results of revascularization in patients with severe left ventricular dysfunction. J Thorac Cardiovasc Surg 2000;1 19(3): $550-557$.

24 Herlitz J, Karlson BW, Sjoland II, Brandrup-Wognsen G, Haglid M, Karlsson T, Caidahl $K$. Long term prognosis after CABG in relation to preoperative left ventricular ejection fraction. Int J Cardiol 2000;72(2):163-171.

25 Tjan TD, Kondruweit M, Scheld HH, Roeder N, Borggrefe M, Schmidt C, Schober O, Deng MC. The bad ventricle: revascularization versus transplantation. Thorac Cardiovasc Surg 2000;48(1):9-14.

26 Argenziano $M$, Spotnitz HM, Whang $W$, Bigger JT Jr, Parides $M$, Rose E $\Lambda$. Risk stratification for coronary bypass surgery in patients with left ventricular dysfunction: analysis of the coronary artery bypass grafting patch trial database. Circulation 1999;100(19 suppl):II1 19-124.

27 Samady H, Elefteriades JA, Abbott BG, Mattera JA, McPherson CA, Wackers IJ. Failure to improve left ventricular function after coronary revascularization for ischemic cardiomyopathy is associated with worse outcome. Circulation 1999;100(12):1298- 1304.

28 Koutlas IC, Elbeery JR, Williams JM, Moran JF, Francalancia NA, Chitwood WR Jr. Myocardial revascularization in the elderly using beating heart coronary artery bypass surgery. Ann Thorac Surg 2000;69(4):1042-1047.

29 Akiyama K, Ogasawara K, Inoue T, Shinodou S, Okumura H, Negishi N, Sezai Y. Myocardial revascularization cardiopulmonary bypass in patients with operative risk factors. Ann Thorac Surg 1999;5(1):31-35.

30 Griffith BP, Hardesty RL, Trento A, Kormos RL, Bahnson ITT. Cardiac transplantation: emerging from an experiment to a service. Ann Surg 1986; 204: 308-314.

31 Evans RW, Mannien DL, Garrison LP, Maier AM. Donor availability as the primary determinant of the future of heart transplantation. JAMA 1986; 255:1892-1898.

32 Mcmanus RP, O'Hair DP, Bretzinger JM, Schweiger JS, Siegel R, Breen TJ, Olinger GN. Patients who died awaiting heart transplantation. J Heart Lung Transplant 1993;12:159-172.

33 Kauffman HM, McBride MA, Shield CF, Daily OP, Wolf JS, Kirklin JK. Determinants of waiting time for heart transplants in the United States. J Heart Lung Transplant 1999;18:414-419.

34 Keon WJ. Heart transplantation in perspective. J Card Surg 1999;14(2):147-151.

35 Hosenpud JD, Bennett LE, Keck BM, Fiol B, Boucek MM, Novick RJ. The registry of the society for heart and lung transplantation: Sixteenth official report - 1999.

36 Batista RJ, Santos JL, Takeshita N, Bocchino L, Lima PN, Cunha MA. Partial left ventriculectomy to improve left ventricular function in end-stage heart disease. J Card Surg 1996;11(2):96-97. 
37 Garcia JM, Barril J, Manapat A, Lopez (G, Luna B. Partial left ventriculectomy (Batista procedure) in the treatment of dilated cardiomyopathy: Makati Medical Center Philippine experience. I Card Surg 1999;14(3):211-213.

38 Kawaguchi AT, Bergsland J, Ishibashi-Ueda II, Ujiie T, Shimura S, Koide S, Salerno TA, Batista RJ. Partial left ventriculectomy in patients with dilated failing ventricle. J Card Surg 1998;13(5):335-342.

39 Etoch SW, Koenig SC, Laureano MA, Cerrito P, Gray L $\Lambda$, Dowling RD. Results after partial left ventriculectomy versus heart transplantation for idiopathic cardiomyopathy. J Thorac Cardiovasc Surg 1999;1 17(5):952-959.

40 Bank AJ, Mir SH, Nguyen DQ, Bolman RM 3rd, Shumway SJ, Miller LW, Kaiser DR, Ormaza SM, Park SJ. Effects of left ventricular assist devices on outcomes in patients undergoing heart transplant, Ann Thorac surg 2000;69(5):1369-1374.

41 Di Bella I, Pagani F, Banfi C, Ardemagni E, Capo E, Klersy C, Vigano M. Results with the Novacor assist system and evaluation of long-term assistance. Eur I Cardiothorac Surg 2000;18(1):112-116.

42 Mussivand $\mathrm{T}$. Mechanical circulatory devices for the treatment of heart failure. J Card Surg 1999;14(3):218-228.

43 Jaski BE, Lingle RJ, Kim J, Branch KR, Goldsmith R, Johnson MR, Lahpor JR, Icenogle TB, Pina I, Adamson R, Favrot LK, Dembitsky WP. Comparison of functional capacity in patients with end-stage heart failure following implantation of a left ventricular assist device versus heart transplantation: results of the experience with left ventricular assist device with exercise trial. J Heart Lung Transplant 1999;18(11):1031-1040.

44 Deng MC, Wilhelm M, Weyand M, Hammel D, Kerber S, Breithardt G, Scheld HH. Long-term left ventricular assist device support: a novel pump rate challenge exercise protocol to monitor native left ventricular function. J Heart Lung Transplant 1997;16(6):629-635.

45 Kaufman R, Reul $\mathrm{H}$, Rau G. The Helmholtz total artificial heart labtype. Artif Organs $1994 ; 18: 537-542$.

46 Hammar C, Schutz A, Pratschke J, Breuer M, Engelhardt M, Brandl U, Saumweber D, Babic R, Kemkes BM, Reichart B. Bridging to transplant: allogeneic heart transplantation after xenografting. J Heart Lung Transplant 1992;11(4 Pt 2):s182-188.

47 Zhang Z, Lazarovits A, Gao Z, Garcia B, Jiang J, Wang J, Xing IJ, White M, Zhong R. Prolongation of xenograft survival using monoclonal antibody CD45RB and cyclophosphamide in rat-to-mouse kidney and heart transplant models. Transplantation 2000;69(6):1137-1146.

48 Hastillo A, Hess ML, Heart xenografting: a route not trod. J Heart Lung Transplant 1993;12(1 Pt 1):3-4.

49 Copeland JG, Arabia FA, Smith RG, Sethi GK, Nolan PE, Banchy ME. Arizona experience with CardioWest Total Artificial Heart bridge to transplantation. Ann Thorac Surg 1999;68(2):756-760.

50 Copeland JG. Current status and future directions for a total artificial heart with a past. Artif Organs 1998;22(11):998-1001.

51 Carpentier A, Chachques JC, Acar C, Relland J, Mihaileanu S, Bensasson D, Kieffer JP, Guibourt P, Tournay D, Roussin I, Grandjean PA. Dynamic cardiomyoplasty at seven years. J Thorac Cardiovasc Surg, 1993; 106: 42-52.

52 Moreira LFP, Stolf NAG, Bocchi EA et al. Clincal and left ventricular function outcomes up to five years after dynamic cardiomyoplasty. J Thorac Cardiovasc Surg 1995; 109:353-363. 
53 Chachques JC, Radermercker M, Tolan MJ, Fischer EIC, Grandjean PA, Carpentier A, Aortomyoplasty counterpulsation: Experimental results and early clinical experience. Ann Thorac Surg, 1996; 61: 420-425.

54 Pochettino A, Anderson DR, Hammond RL,. Skeletal muscle ventricles: a promising treatment option for heart failure. J Cardiac Surg 1991; 6(suppl.):145-153.

55 Guldner NW, Klapproth P, Grossherr M, Stephan M, Rumpel E, Noel R, Sievers HH. Clenbuterol-supported dynamic training of skeletal muscle ventricles against systemic load: a key for powerful circulatory assist? Circulation. 2000 May 9;101(18):2213-9.

56 Wstaby S. Non-transplant surgery for heart failure. Heart 2000;83(5):603-610.

57 Arnolda LF, Llewellyn-Smith IJ, Minson JB. Animal models of heart failure. Aust N Z J Med 1999;29(3):403-409.

58 Arnolda LF, Liewellyn-Smith IJ, Minson JB. Animal models of heart failure. Aust NZ J Med 1999;29:403-409.

59 Hasenfuss G. Animal models of human cardiovascular disease, heart failure and hypertrophy. Cardiovasc Res 1998;39:60-76.

60 Power JM, Tonkin AM. Large animal models of heart failure. Aust $N$ Z J Med 1999;29(3):395-402.

61 Winslow RL, Rice J, Jafri S, Marban E, O'Rourke B. Mechanisms of altered excitationcontraction coupling in canine tachycardia-induced heart failure, II: model studies. Circ Res 1999; 19;84(5):571-586.

62 Sabbah HN, Stein PD, Kono T, Gheorghiade M, Levine TB, Jafri S, Hawkins ET, Goldstein $S$. A canine model of chronic heart failure produced by multiple sequential coronary microembolizations. Am J Physiol 1991;260(4 Pt 2):H1379-1384.

63 Desjardins S, Mueller RW, Cauchy MJ. A pressure overload model of congestive heart failure in rats. Cardiovasc Res 1988;22(10):696-702.

64 Flaim SF, Minteer WJ, Nellis SH, Clark DP. Chronic arteriovenous shunt: evaluation of a model for heart failure in rat. Am J Physiol 1979;236(5):H698-704.

65 Muders F, Friedrich E, Luchner A, Pfeifer M, Ickenstein G. Hemodynamic changes and neurohemoral regulation during development of congestive heart failure in a model of epinephrin-induced cardiomyopathy in conscious rabbits. J Card Fail 1999; 5(2):109-116.

66 Tofovic SP, Kusake H, Rominski B, Jackson EK. Caffeine increases renal renin secretion in a rat model of genetic heart failure. J Cardiovasc Pharmacol 1999;33(3):440-450.

67 Nishio R, Matsumori A, Shioi T, Wang W, Yamada T, Ono K, Sasayama S. Denopamine, a beta $I$ - adrenergic agonist, prolongs survival in a murine model of congestive heart failure induced by viral myocarditis: suppression of tumor necrosis factor-alpha production in the heart. J Am Coll Cardiol 1998;32(3):808-815.

68 Genao A, Seth K, Schmidt U, Carles M, Gwathmey JK. Dilated cardiomyopathy in turkeys: an animal model for the study of human heart failure. Lab Anim Sci 1996;46(4):399-404.

69 Chen HH, Schirger JA, Chau WL, Jougasaki M, Lisy O, Redfield MM, Barclay PT, Burnett JC Jr. Renal response to acute neutral endopeptidase inhibition in mild and severe experimental heart failure. Circulation 1999;100(24):2443-2448.

70 Amstrong PW, Stopps TP, Ford SE, de Bold AJ, Rapid ventricular pacing in the dog: pathophysiologic studies of heart failure. Circulation 1986;74:1075-1084.

71 Wilson JR, Douglas P, Hickey WF, et al. Experimental congestive heart failure produced by rapid ventricular pacing in the dog; cardiac effects. Circulation 1987;75:857-867. 
72 Whipple GH, Sheffield LT, Woodman EG, Thoephilis C, Iriedman S. Reversible congestive heart failure due to rapid stimulation of the normal heart. Proc New ling Cardiovasc Soc 196;20:39-40.

73 Preffer MA, Preffer JM, Fishbein MC, et al. Myocardial infarct size and ventricular function in rats. Circ Res 1979;44:503-512.

74 Liwin SE, Kats SE, Morgan JP, Douglas PS. Serial echocardiographic assessment of left geometry and function after large myocardial infarction in the rat. Circulation 1994;89:345-354.

75 Kajstura J, Zhang X, Reiss K, et al. Myocyte cellular hyperplasia and myocyte cellular hyperthrophy contribute to chronic ventricular remodeling in coronary artery narrowing-induced cardiomyopathy in rats. Circ Res 1994;74:383-400.

76 Van der Meer JJ, Reneman RS. An improved technique to induce a standardized functional stenosis of coronary artery. Eur Surg Res 1972;4:407-418.

77 Hosko MJ, Gross GJ, Warltier DC. Technique for precise, graded arterial stenosis and occlusion. Bas Res Cardiol 1977;72:651-659.

78 Zietkiewicz M, Perek B, Meyns B, Mesotten L, Dispersyn G, Nishimura Y, Flameng W. Chronic heart failure model induced by coronary embolization in sheep. Int J Atrif Organs 1999;22(7):499-504.

79 Sharov VG, Goussev A, Lesch M, Goldstein S, Sabbah HN. Abnormal mitochondrial function in myocardium of dogs with chronic heart failure. J Mol Cell Cardiol 199830(9):1757-1762.

80 Gengo PJ, Sabbah HN, Steffen RP, et al. Myocardial beta adrenoceptor and voltage-sensitive calcium chnnel changes in a canine model of chronic heart failure. J Mol Cell Cardiol 1992;24:1361-1369.

81 Gupta RC, Shimoyama H, Tanimura M, et al. SR Ca2+ $-\Lambda$ TPase activity and expression in ventricular myocardium of dogs with heart failure. Am J Physiol 1997;273:H12-H18.

82 McCullagh WH, Covell JW, Ross J Jr. Left ventricular dilatation and diastolic compliance changes during chronic volume overloading. Circulation 1972;45:943-951.

83 Kleavland JP, Kussmaul WG, Vinciguerra T, Diters R, Carabello BA, Volume overload hyperthrophy in closed-chest model of mitral regurgitation. $\Lambda \mathrm{m} J$ Physiol 1988;254:H1304-H1041.

84 Liu SK, Magid NR, Fox PR, Goldfine SM, Borer JS. Fibrosis, myocyte degeneration and heart failure in chronic experimental aortic regurgitation. Cardiology 1998;90(2): 101-109.

85 Yoshimura R, Sato T, Kawada T, Shishido T, Inagaki M, Miyano H, Sugimachi M, Sunagawa $\mathrm{K}$. Increased brain angiotensin receptor in rats with chronic high-output heart failure. J Card Fail 2000;6(1):66-72.

86 Flaim SF, Minteer WJ, Nellis SH, Clark DP. Chronic arteriovenous shunt: evaluation of a model for heart failure in rat. Am J Physiol 1979;236(5):H698-704.

87 Porter CB, Walsh RA, Badke FR, O'Rourke RA. Differential effects of diltiazem and nitroprusside on left ventricular function in experimental chronic volume overload. Circulation 1983;68:685-692.

88 Kiss E, Ball NA, Kranias EG, Walsh RA. Differential changes in cardiac phospholamban and sarcoplasmic reticulum Ca2+-ATPase protein levels. Effects on $\mathrm{Ca} 2+$ transport and mechanics in compensated pressure-overload hypertrophy and congestive heart failure. Circ Res 1995;77:759-764.

89 Koide M, Nagatsu M, Zile MR, Hamawaki M, Swindle MM, Keech G, DeFreyte BS, Tagawa $H$, Cooper G, Carabello A. Premorbid determinants of left ventricular 
dysfunction in a novel model of gradually induced pressure werloat in the adult canine. Circulation 1997;95:1601-1610.

90 Siri FM, Krueger I, Nordin C, Ming Z, Aronson RS. Depressed intracellular calcium transients and contraction in myocytes from hypertrophied and failing guinea pig hearts. Am J Phys 1991;261:H514-1530.

91 Shah HR, Vaynblat M, Ramdev G, Cumningham JN Jr, Chiavarelli M. Experimental cardionyopathy as a model of chronic heart failure. J Invest Surg 1997;10(6):387-396.

92 Muders F, Friedrich E, Luchner $\Lambda$, Preifer M, Ickenstein G, Hamelbeck B, Riegger $G \Lambda$, Elsner D. Hemodynamic changes and neurohumoral regulation during development of congestive heart failure in a model of epinephrine-induced cardiomyopathy in conscious rabbits. J Card Fail 1999;5(2):109-116.

93 Lucas C, Cheriex EC, Van der Veen FH, Habets J, Van der Nagel T, Penn OC, Wellens IIJ. Imipramine induced heart failure in the dog: $\Lambda$ model to study the effect of cardiac assist devices. Cardiovasc Res 1992;26:804-809.

94 Edwards JG, Lyon GE, Micales BK, Malhotra $\Lambda$, Factor S, Leinwand LA. Cardiomyopathy in transgenic myf5 mice. Circ Res 1996;78:379-387.

95 Hall DG, Morley GE, Vaidya D, Ard M, Kimball TR, Witt S $\Lambda$, Colbert MC. Early onset heart failure in transgenic mice with dilated cardiomyopathy. Pediatr Res 2000;48(1):36-42.

96 Rockman HA, Ono S, Ross RS, et al. Molecular and physiological alternation in murine ventricular dysfunction. Proc Natl Acad Sci USA 1994;91:2694-2698.

97 Yamada T, Matsumori A, Wang WZ, Ohashi N, Shiota K, Sasayama S. Apoptosis in congestive heart failure induced by viral myocarditis in mice. Heart vessels 1999;14(1):29-37.

98 Doi R, Masuuyama T, Yamamoto K, Doi Y, Mano T, Sakata Y, Ono K, Kuzuya T, Hirota S, Koyama T, Miwa T, Hori M. Development of different phenotypes of hypertensive heart failure: systolic versus diastolic failure in Dahl salt-sensitive rats. I Hypertens $2000 ; 18(1): 111-120$.

99 Bajusz L. Hereditary cardiomyopathy: a new disease model. Am Heart J 1969:686-696.

100 Jasmin G, Proschek L. Hereditary polymyopathy and cardiomyopathy in Syrian hamster. I. Progression of heart and skeletal muscle lesions in the UM-X7.1 line. Muscle Nerve 1982;5:20-25.

101 Noguchi K, Matsuzaki T, Koyama T, Itomine T, Sakanashi M. Comparison of haemodynamic responses to cilnidipine and nicardipine in an experimental model of acute congestive heart failure. Clin Exp Pharmacol Physiol 1998;25(7-8):541-547.

102 Pichardo J, Palace V, Farahmand F, Singal PK. Myocardial oxidative stress changes during compensated right heart failure in rats. Mol Cell Biochem 1999;196(1-2)51-57.

103 De Jesus JR. Breve considerationes sobre un caso de herida penetrante del corazon. BolAssoc Med (PR) 1931;23:380-382.

104 Beck DS, The development of new blood supply to the heart by operation. Ann Surg 1935;102:229-232.

105 Petrovsky BV. Surgical treatment of cardiac aneurysms. J Cardiovasc Surg 1996;2:87-91.

106 Kantrowitz A, McKinnon WM. The experimental use of the diaphragm as an auxiliary myocardium. Surg Forum 1959; 9: 266-268.

107 Termet H, Chalencom JL, Estoir E, et al. Transplantation sur la myocarde d'un muscle strie par excite par pacemaker Ann. Chir Thorac Cardio 1966;5:568-571.

108 Salmons S, Vrbova G. The influence of activity on some contractile characteristics of mammalian fast and slow muscle. J Physiol Lond 1969; 201:535-549. 
109 Salmons S, I Ienriksson J. The adaptive response of skeletal muscle to increased use. Muscle and Nerve 1981; 4:94-105.

110 Dewar ML., Dtinkwater DC, Wittnich C, Chiu RC. Synchronously stimulated skeletal muscle graft for myocardial repair. J Thorac Cardiovasc Surg 1984; 87:325-331.

11 Carpentier A, Chachques JC. Myocardial substitution with a stimulated skeletal muscle: first successful clinical case. Lancet 1985; 1:1267.

112 Chachques JC, Grandjean PA, Carpentier A. Latissimus dorsi dynamic cardiomyoplasty. Ann Thorac Surg 1989; 47:600-604.

113 Magoveren GJ, Heckler $F R$, Park $S B$, et al. Paced skeletal muscle for dynamic Cardiomyoplasty. Ann Thorac Surg 1988;45:614-619.

114 Delahaye F, Jegaden O, Montagna P, et al. Latissimus dorsi Cardiomyoplasty in severe congestive heart failure: The Lyon experience.) Cardiac Surg 1991;6(suppl.):106-112.

115 Carpentier $\Lambda$, Chachques JC, $\Lambda$ car C, Relland J, Mihaileanu S, Bensasson D, Kieffer JP, Guibourt P, Toumay D, Roussin I, Grandjean PA. Dynamic cardiomyoplasty at seven years. J Thorac Cardiovasc Surg, 1993; 106: 42-52.

116 Adams RJ, Schwartz A. Comparative mechanisms for contraction of cardiac and skeletal muscle. Chest 1989; 78(suppl.): 123-129.

117 Johnson MA, Polgar J, Weightman D, Appleton D. Data on the distribution of fibre types in thirty-six human muscles. An autopsy study. J. Neurol. Sci 1973; 18: 121-129.

118 Sola OM, Haines LC, Kakulas BA et al.Comparative anatomy and histochemistry of human and canine latissimus dorsi muscle. J Heart Transplant 1990; 9:151-159.

119 Lucas CM, Dubclaar ML, Van der Veen FH et al. A new stimulation protocol for cardiac assist using the latissimus dorsi muscle. Pace 1993; 16:2012-2021.

120 Van der Veen FH, Davidse JHL. Structural alternations in the latissimus dorsi muscles in three patients two years after a cardiomyoplasty procedure. Eur. J. C. P. E. 1996; 6: 150 (abstract).

121 Davidse JHL, Van der Veen FH, Lucas CMHB, Penn OCKM, Daemen MJAP, Wellens IIJ]. Structural alternations in the latissimus dorsi muscles in three patients more than 2 years after a cardiomyoplasty procedure. Eur Heart J 1998;19:310-318.

122 Davidse JHL, Lucas CMHB, Daemen MJAP, Penn OCKM, Van der Veen FH. Skeletal muscle adaptation in patients with severe heart failure: Implication for aorto-and cardiomyoplasty. Basic Appl. Myol.1997;7(1):23-30.

123 Lucas CM, Van der Veen FH, Cheriex EC, et al. Long term follow-up (12 to 35 weeks) after dynamic cardiomyoplasty. J. Am Coll Cardiol 1993;22:758-767.

124 Millner RWI, Burroms M, Pearson I, Pepper JR. Dynamic cardiomyoplasty in chronic left ventricular failure: an experimental model. Ann Thorac Surg 1993;55:493-501.

125 Cheng W, Michele JJ, Spinale PG, et al. Effects of cardiomyoplasty on biventricular function in canine chronic heart failure. Ann Thorac Surg 1993;55:893-901.

126 Lee KF, Dignan RJ, Parmer JM, et al. Effects of dynamic cardiomyoplasty on left ventricular performance and myocardial mechanics in dilated cardiomyopathy. I Thorac Cardiovasc Surg 1991;102:124-131.

I27 Manion JD, Buckman PD, Mango MG, Dimco FD. Collateral blood flow from skeletal muscle to normal myocardium. J Surg Res 1992;53:578-587.

128 Bailey WF, Mango MG, Buckman PD. et al. Chronic stimulation enhanced extramyocardial collateral blood flow after a cardiomyoplasty. Ann Thorac Surg 1993; 56:1045-1053.

129 Capouya ER, Gerber RS, Drinkwater DC, et al. Girdling effect of nonstimulated cardiomyoplasty on left ventricular function. Ann Thorac Surg 1993;56:867-871. 
130 Kaulbach HG, Van der Veen FH, Schreuder JJ, et al. Iynamic cardiomyoplasty in chronic cardiac overload [Abstract]. Circulation 1995;92:I-379.

131 Magoveren JA, Magoveren GJ, Maher TD, et al. Operation for congestive heart failure: transplantation, coronary artery bypass and cardiomyoplasty. Amn Thorac Surg 1993;56:418-424.

132 Moreira LFP, Stolf NAG, Bocchi EA, et al. Clinical and left ventricular function outcomes up to five years after dynamic cardiomyoplasty. I Thorac Cardiovasc Surg 1995; 109:353-363.

133 Bellotti G, Moraes A, Bocchi E, et al. Late effects of cardiomyoplasty on left ventricular mechanics and diastolic filling. Circulation 1993;88(part 2):304-308.

134 Kass Dk, Baughman KL, Pak PH, et al. Reverse remodeling from cardiomyoplasty in human heart failure. Circulation 1995;91:2314-2318.

135 Schreuder JJ, Van der Veen, Van der Velde ET, et al. Beat-to-beat analysis of left ventricular pressure-volume relation and stroke volume by conductance catheter and aortic model flow in cardiomyoplasty patients. Circulation 1995;91:2010-2017.

136 Furnay AP, Magovern JA, Christlieb IY, et al. Clinical cardiomyoplasty: preoperative factors associated with outcome. Ann Thorac Surg. 1992;54:1139-1143.

137 Mueller H, Ayres SM, Conclin EF, Giannelli SJR, Mazzara JT, Grace WT, Nealon TF. The effects of intra-aortic counterpulsation on cardiac performance and metabolism in shock associated with acute myocardial infarction. J Clin Invest 1971; 50: 1885-1900.

138 Kerber RE, Marcus ML, Ehrhardt J, Abbovd FM. Effect of intra-aortic balloon counterpulsation on the motion and perfusion of acutely ischemic myocardium. Circulation 1976; 53: 853-859.

139 Fuchs RM, Brin KP, Brinker JA, Guzman PA, Hevzer RR, Yin FCP. Augmentation of regional coronary blood flow by intra-aortic balloon counterpulsation in patients with unstable angina. Circulation 1983; 68: 117-123.

140 Freed PS, Wasfie T, Zado B, Kantrowitz $A$. Intra-aortic balloon pumping for prolonged circulatory support. Am J Cardiol 1988; 61: 554-557.

141 Chachques JC, Grandjean PA, Fischer EIC, Latremouille C, Jebara VA, Bourgeois I, Carpentier A. Dynamic Aortomyoplasty to assist left ventricular failure. Ann Thorac Surg 1990;49:225-230.

142 Pattison CW, Cumming DVE, Williamson A, Clayton-Jones DG, Dumm MJ, Goldspink $G$, Yacoub M. Aortic counterpulsation for up to 28 days with autologous latissimus dorsi in sheep. J Thorac Cardiovasc Surg 1991;102:766-773.

143 Mesana TG, Mouly A, Caus Th, Bauer S, Collard F, Monties IR. Preliminary clinical experience with descending dynamic aortomyoplasty. EUR. J. C. P. E. 1996; 6(supp): 150.

144 Flum DR, Cernaianu AC, Meada R, Lee LA, Salartash K, Grosso MA, Weiss RL, Cilley JH, Delrossi AJ. Descending thoracic aortomyoplasty: a technique for clinical application. Ann Thorac Surg 1996; 61: 93-98.

145 Chachques JC, Haab F, Cron C, Fischer EIC, Grandjean PA, Bruneval P, Acar C, Jebara VA, Fontaliran F, Carpentier A. Long-term effects of dynamic aortomyoplasty. Ann Thorac Surg 1994; 58: 128-134.

146 Lazzara RR, Trumble DR, Magovern JA. Chronic counterpulsation with descending thoracic aortomyoplasty improved cardiac function in animals with heart failure. $J$ Heart Lung Transplant 1994; 13: 652-660.

147 Chachques JC, Radermercker M, Tolan MJ, Fischer EIC, Grandjean PA, Carpentier A. Aortomyoplasty counterpulsation: Experimental results and early clinical experience. Ann Thorac Surg 1996; 61: 420-425. 
148 Mesana TG, Mouly-Bandini A, Ferzoco SJ, Collard F, Caus T, Reul RM, Monties JR, Schoen FJ, Cohn LH. Dynamic aortonyoplasty: clinical experience and thoracoscopic surgery feasibility study.J. Card Surg 1998; 13:60-69.

149 Swan HCJ, Ganz W, Forrester J et al. Catheterization of the heart in man with the use of a flow-directed balloon-tipped catheter. N Engl J Med 1970;283:447-451.

150 Ginosar Y, Sprung CL. The Swan-Ganz catheter: Twenty-five years of monitoring. Crit Care Clin 1996;12:771-776.

151 Stetz CW, Miller RG, Kelly GE, Raffin TA. Reliability of the thermodilution method in the determination of cardiac output in clinical practice. Am Rev Respir Dis. 1982;126: 1001-1004.

152 Manny J, Grindlinger GA, Dennis RC, Weisel RD, Hechtman HB. Myocardial performance curves as guide to volume therapy. Surg Gynecol Obstet 1979; 149: 863-873.

153 Sibbald WJ, Driedger A^, Myers ML, Short AIK, Wells GA. Biventricular function in the adult respiratory distress syndrome: hemodynamic and radionuclide assessment, with special emphasis on right ventricular function. Chest 1983;84:126-134.

154 Wo CCJ, Shoemaker WC, Appel PL, Bishop MH, Kram HB, Hardin E. Unreliability of blood pressure and heart rate to evaluate cardiac output in emergency resuscitation and critical illness. Crit Care Med 1993;21:218-223.

155 Geoedje O, Hoeke K, Lichtwarck-Aschoff M, Faltchauser A, Lamm P, Reichart B. Continuous cardiac output by femoral arterial thermodilution calibrated pulse contour analysis: comparison with pulmonary arterial thermodilution. Crit Care Med $1999 ; 27(11): 2407-2412$.

156 Urzua J, Salinas C, Cipriano A, Guarini M, Lema G, Canessa R. Estimation of ventricular volume and elastance from the arterial pressure waveform. J Clin Monit Comput 1998;14(3):177-181.

157 Hirschi MM, Binder M, Gwechenberger M, Herkner H, Bur A, Kittler H, Laggner AN. Noninvasive assessment of cardiac output in critically ill patients by analysis of the finger blood pressure waveform. Crit Care Med 1997;25(11):1909-1914.

158 Tadaoka S, Kagiyama M, Hiramatsu O, Ogasawara Y, Tsujioka K, Wada Y, Sawayama T, Kajiya F. Accuracy of $20-\mathrm{MHz}$ Doppler catheter coronary artery velocimetry for measurement of coronary blood flow velocity. Cathet Cardiovasc Diagn 1990;19(3): 205-213.

159 Nagueh SF. Noninvasive evaluation of hemodynamics by Doppler echocardiography. Curr Opin Cardiol 1999;14(3):217-224.

160 Rodevan O, Bjornerheim R, Ljosland M, Maehle J, Smith HJ, Ihlen H. Left atrial volumes assessed by three- and two-dimensional echocardiography compared to MRI estimates. Int J Card Imaging 1999;15(5):397-410.

161 Nadkarni SK, Boughner DR, Drangova M, Fenster A. Three-dimensional echocardiography: assessment of inter-and intra-operator variability and accuracy in the measurements of left ventricular cavity volume and myocardial mass. Phys Med Biol 2000;45(5):1255-1273.

162 De Castro S, Pandian NG. Three-dimensional echocardiography: clinical relevance and application. Am J Cardiol 1998;81(12A):96G-102G.

163 Sonka M, Liang W, Kjanani P, Allan J, DeJong S, Kerber R, Mckay C. Intracardiac echocardiography: computerized detection of left ventricular borders. Int J Card Imaging 1998;46(6):397-411.

164 Heusch A, Koch JA, Krogmann ON, Korbmacher B, Bourgeois M. Volumetric analysis of the right and left venticle in a porcine heart model: comparison of three-dimensional 
echocardiography, magnetic resonance imaging and angiocardiography. Fur I Ultrasound $1999 ; 9(5): 245-255$.

165 Jiang L, Morrissey R, Handschumacher MD, Vazquez de Prada $3 \Lambda$, lle ], Picard MH, Weyman AE, Levine RA. Am heart J 1996;131:553-559.

166 Chen Ch, Nevo E, Fetics B, Nakayama M, Pak PH, Maughan WL, Kass DA. Comparison of continuous left ventricular volumes by transthoracic two-dimensional digital echo quantification with simultaneous conductance catheter measurements in patients with cardiac disease. Am J Cardiol 1997;80(6):756-761.

167 Gorcsan J III, Denault A, Mandarino WA, Pinsky MR. Left ventricular pressure-volumes relations with transesophageal echocardiographic automated border detection: comparison with conductance-catheter technique. Am Heart J 1996;131 (3):544-552.

168 Balestrini L, Fleishman C, Lazoni L, Kisslo J, Resai Bengur $\Lambda$, Sanders SP, Li JS. Real-time 3-dimensional echocardiography evaluation of congenital heart disease. J Am Soc Echocardiogr 2000;13(3):171-176

169 Burkhoff ZD, Van der Valde E, Kass D, Baan J, Maughan W, Sagawa K. Accuracy of volume measurements by conductance catheter in isolated, ejecting canine hearts. Circulation 1985;72(2):440-447.

170 Dritsas A, Joshi J, Webb SC, Athanassopoulos G, Oakley CM, Nihoyannopoulos P. Beatto beat variability in stroke volume during VVI pacing as predictor of hemodynamic benefit from DDD pacing. PACE 1993;16:1713-1718.

171 Gepstein L, Hayam G, Ben-Haim SA. A novel method for nonfluoroscopic catheter-based electroanatomical mapping of the heart. In-vitro and in-vivo accuracy results. Circulation 1997;95:1611-1622.

172 Gepstein L, Hayam G, Shpun S, Ben-Haim SA. Homodynamic evaluation of heart with a nonfluoroscopic electroanatomical mapping technique. Circulation 1997;96:3672- 3680.

173 Kornowski R, Hong MK, Gepstein L, Goldstein S, Ellahham S, Ben-Haim S $\Lambda$, Leon MB. Preliminary animal and clinical experiences using an electroanatomical endocardial mapping procedure to distinguish infarced from healthy myocardium. Circulation 1998;98:1116-1124.

174 Gepstein L, Evans SJ. Electroanatomical mapping of the heart: Basic concepts and implications for the treatment of cardiac arrhythmias. PACE 1998;21:1268-1278.

175 Nademanee K, Kosar EM. A nonfluoroscopic catheter-based mapping technique to ablate focal ventricular tachycardia. PACE 1998;21:1442-1447.

176 Kornowski R, Hong MK, Leon MB. Current perspectives on direct myocardial revascularization. Am J Cardiol 1998;81(7A):44E-48E.

177 Gepstein L, Goldin A, Lessick J, Hayam G, Shpun S, Schwartz Y, Hakim G, Shofti R, Turgeman A, Kirshenbaum D, Ben-Haim SA. Electromechanical characterization of chronic myocardial infarction in the canine coronary occlusion model. Circulation 1998;98:2055-2064.

178 Lorusso R, Van der Veen FH, Schreuder JJ, Bolotin G, Kaulbach HG, Frietman R, Habets Jo, Van der Nagel $T$, Wellens HJ. Hemodynamic effects in acute cardiomyoplasty of different wrapped muscle activation times as measured by pressure/volume relation. J Card Surg 1996;11:217-225.

179 Schreuder JJ, Van der Veen, Van der Velde ET, et al. Beat-to-beat analysis of left ventricular pressure-volume relation and stroke volume by conductance catheter and aortic model flow in cardiomyoplasty patients. Circulation 1995;91:2010-2017. 


\section{Acute and chronic heart dilatation model- induced in goats by carotid jugular A-V shunt}

Gil Bolotin, Roberto Lorusso, Hans Kaulbach, Jan Schreuder, Gideon Uretzky, Frederik van der Veen

Presented at the Fourth World Congress on Heart Failure, Jerusalem, 1996. The Journal of Heart Failure, 1996; 3: 591. 


\begin{abstract}
Objectives: Congestive heart failure is characterized by impaired quality of life and markedly shortened life expectancy. There are currently many medical and surgical modalities that aim to treat patients with heart failure, which implies the need for an animal heart failure model for the development and testing these therapeutic modalities. The purpose of this study was to evaluate the acute and chronic effect of carotid-jugular A-V shunt in goat model of heart dilatation.

Methods: After cross clamping of the left carotid artery, an end-to-side anastomosis of ten $\mathrm{mm}$ in diameter was performed between the free end of the vein and the side of the artery. A dual-micromanometer transducer conductance catheter was used to determine the pressure-volume loops of the left ventricle. The acute and chronic hemodynamic changes were measured at baseline, immediately after the creation of the shunt, and eight weeks later.

Results: During and at the end of the eight weeks none of the animals showed clinical signs of heart failure or distress. There was an immediate rise in LVEDV (104 $\pm 27 \mathrm{ml}$ ) as compared to the baseline volume ( $75 \pm 26$ ), and eight weeks later the LVEDV reached $111 \pm 22$.

Conclusions: The carotid-jugular A-V shunt in goats causes significant immediate and long-term heart dilatation, without signs of heart failure. This model is reproducible with low animal mortality and is appropriate for the study of cardiomyopalsty.
\end{abstract}




\section{INTRODUCTION}

Congestive heart failure is characterized by impaired quality of life and markedly shortened life expectancy (1). Optimal medical treatment of patients with congestive heart failure improves the quality of life and survival rates, but mortality is still unacceptably high (2). There are currently many medical and surgical modalities that aim to treat patients with heart failure, which implies the need for an animal heart failure model for the development and testing of these therapeutic modalities. A variety of animal heart failure models have been developed, including those in which heart failure is induced via drugs (3), rapid ventricular pacing (4), genetic alteration (5), viral sources (6), idiopathic dilated cardiomyopathy (7), coronary arterial embolization (8), pressure overload (9), and volume overload by arterio-venous $(A-V)$ shunt (10). The A-V shunt model induces left heart dilatation that mimics the clinical situation in patients with dilated cardiomyopathy (11).

The purpose of this study was to evaluate the acute and chronic effect of carotid-jugular $\mathrm{A}-\mathrm{V}$ shunt in goat model of heart dilatation.

\section{METHODS}

Experiments were performed in eight female goats, weighing 51 to $80 \mathrm{~kg}$, in accordance with the Guide for the Care and Use of Laboratory Animals published by the National Institutes of Health (NIH publication 86-23, revised 1985).

Dilated cardiomyopathy was induced by a left carotid jugular A-V shunt. The acute and chronic hemodynamic changes were followed for 8 to 10 weeks by three different monitoring modalities: Swan-Ganz catheters measured cardiac output before, immediately after the creation of the A-V shunt, and 8 weeks later; conductance catheters measured left ventricle dimensions and performance before, immediately after the creation of the A-V shunt, and 8 weeks later; and echocardiography was performed before and every two weeks after the A-V shunt operation.

\section{A-V shunt procedure}

General anesthesia was induced by thiopental sodium (Pentothal, Abbott S.P.A., Italy), administered intravenously at $15 \mathrm{mg}$ per $\mathrm{kg}$ body weight, and maintained after endotracheal intubation with oxygen/nitrous oxide (1:2) and 1.5\% Fluothane (Halothane, Zeneca Ltd., Macclesfield, Cheshire, UK). 
During the experiments, the lungs were ventilated with a positive pressure respirator (Harvard, Apparatus Inc., South Natick, Massachusetts) and body temperature was kept constant with a heating mattress. $\triangle$ single dose of 10.000 IU heparin i.v. was administered. Through a cervical incision, the left jugular vein and the left carotid artery were mobilized over a length of approximately $5 \mathrm{~cm}$ and ligated distally. After cross-clamping of the left carotid artery, an end-to-side anastomosis of $10 \mathrm{~mm}$ in diameter was performed between the free end of the vein and the side of the artery, using 6-0 polypropylene nonabsorbable running sutures (Ethicon, Edinburgh, Scotland). Clamps were removed and the patency of the fistula was visually confirmed by the pulsatile filling of the jugular vein.

\section{Heart catheterization}

A dual-micromanometer transducer conductance catheter (7F, Sentron, The Netherlands) was used to determine the volume of the ventricle on-line, by measuring the time-varying electrical conductance of the segments of intraventricular blood (8). The catheter's position along the long axis of the left ventricular cavity via the left femoral artery was set under fluoroscopic guidance and verified by inspection of the segmental conductance signals: a correct position was assumed if the signals of at least the four most distal segments displayed a typical phasic LV volume tracing. A Leycom Sigma-5DF signal conditioner-processor (CardioDynamics, Zoetermeer, The Netherlands) provided the current source and processed the segmental conductance, producing an on-line display of the LV contours, as well as continuous and instantaneous volume signals. The volume signals were combined with the pressure signals on an $X-Y$ oscilloscope to provide an instantaneous and uninterrupted display of pressure-volume loops.

Readings of the volume signal were corrected to account for the parallel conductance caused by tissues surrounding the ventricular cavities. A bolus of $7.5 \mathrm{ml}$ of hypertonic $\mathrm{NaCl}(9 \%)$ was injected into the central venous compartment via the Swan-Ganz catheter. As the bolus mixed with the fluid in the ventricular cavity, its conductivity increased, causing the overall conductance signal to increase while the parallel component remained constant. Endsystolic overall conductance was then plotted as a function of end-diastolic overall conductance during the mixing of the bolus; the conductance was equal to the intersection point between the regression line of these values and the line of identity. 


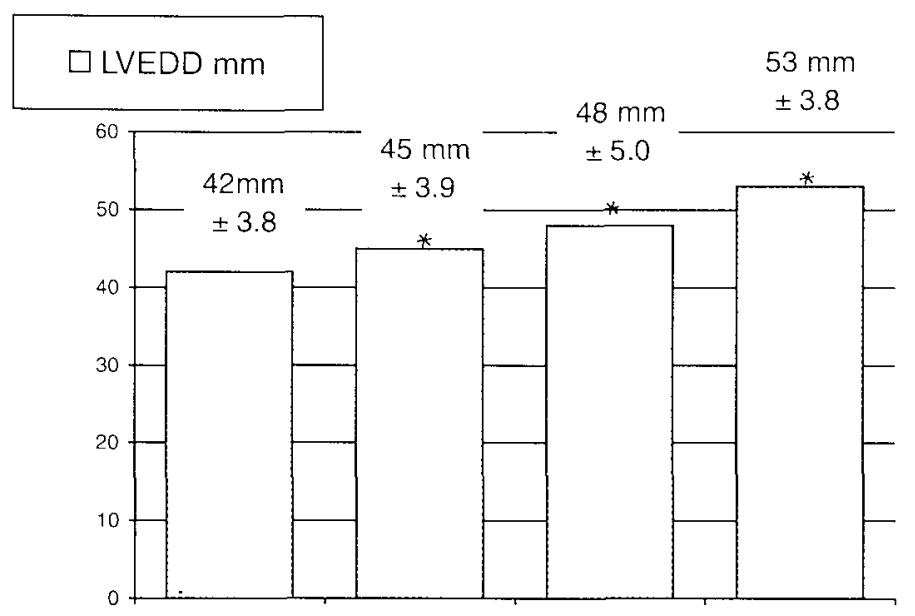

Figure 1. Left ventricular end-diastolic volume assessed by echo cardiography at $2-4$ weeks intervals. ${ }^{*}$ Significant increase as compared to the previous value $(\mathrm{P}<0.05)$.

\section{RESULTS}

During and at the end of the eight weeks none of the animals showed signs of heart failure or distress.

One animal died three weeks after the $\mathrm{A}-\mathrm{V}$ shunt operation most probably due to lung infection.

The carotid-jugular A-V-shunt induced both immediate and long-term hemodynamic changes. The immediate effect of the shunt, an abrupt rise in cardiac output, was observed within 30 minutes after opening of the shunt and measured by Swan-Ganz thermodilution technique: $7.9 \pm 1.9 \mathrm{l} / \mathrm{min}$ compared to the baseline cardiac output of $4.9 \pm 1.3 \mathrm{l} / \mathrm{min}$. Eight weeks after the shunt procedure, an increase in cardiac output was observed in comparison not only to the baseline value but also to the measurements obtained immediately after opening of the shunt, measuring $9.9 \pm 2.7 \mathrm{l} / \mathrm{min}$.

Echocardiography tests revealed a gradual increase in left ventricular enddiastolic diameter, reaching the maximal left ventricle dilatation at 8 weeks (Fig 1).

The acute and chronic hemodynamic changes were demonstrated by pressure-volume loops at baseline, immediately after the shunt, and after 8 weeks (Fig. 2). There was an immediate rise in the LVEDV $(104 \pm 27 \mathrm{ml})$ compared to the baseline volume $(75 \pm 26 \mathrm{ml})$, and eight weeks later the LVEDV reached 111 $\pm 22 \mathrm{ml}$. 


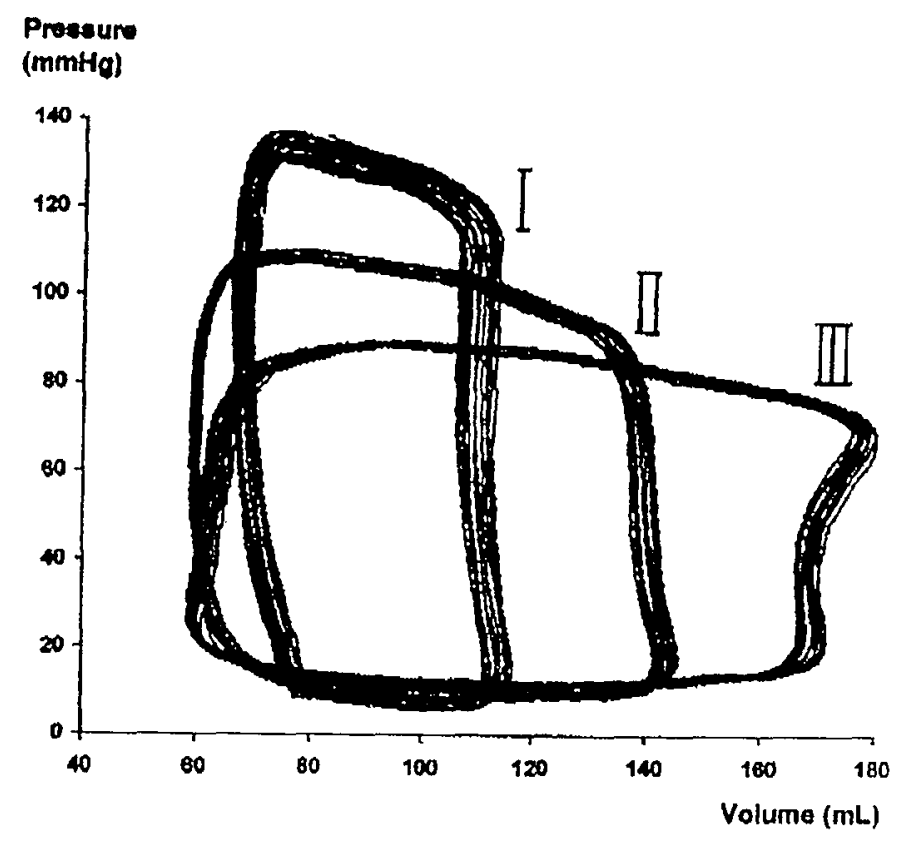

Figure 2. Pressure volume loops before I, 45 minutes after II, and 8 weeks after the creation of the shunt III.

\section{DISCUSSION}

This study demonstrates that the A-V shunt heart failure model is capable of producing stable, immediate and long-term high cardiac output (overload) dilatation. None of the eight goats demonstrated clinical signs of pulmonary congestion. Fortunately, these results are in contrast with the high mortality rates $(77 \%)$ and signs of pulmonary congestion that commonly occur within 24 hours of performing the A-V shunt in small animals, as reported by Flaim and coworkers (10). This discrepancy is probably due to the relatively small $\mathrm{A}-\mathrm{V}$ shunt of approximately $10 \mathrm{~mm}$, used in our study (in goats weighing 51 to $80 \mathrm{~kg}$ ). Moreover, in the present study the animals presented neither clinical nor hemodynamic signs of heart failure, such as tachycardia or an increase in LVEDP, as was observed with inferior vena cava aortic A-V shunt applied in mongrel dogs (12). The successful compensatory mechanism in the goat carotid jugular $\mathrm{A}-\mathrm{V}$ shunt is the reason for the lack of signs of renal failure such as were demonstrated in a rat aortovenocaval fistula by Wegner and coworkers (13). Therefore, the described model should be considered a compensatory overload dilatation model rather than a heart failure model. 
The Swan-Ganz and the conductance catheters that were used to monitor the heart hemodynamic changes immediately after the creation of the $A-V$ shunt revealed interesting results. The abrupt rise in cardiac output generated huge hemodynamic changes. The LVEDV increased from $75 \mathrm{ml}$ to $104 \mathrm{ml}$, while the LVESV reduced from $27 \mathrm{ml}$ to $20 \mathrm{ml}$, together resulting in an abrupt rise in left ventricular ejection fraction (from $57 \%$ to $79 \%$ ) and stroke volume (from $37 \mathrm{ml}$ to $72 \mathrm{ml}$ ). Strangely, no changes in heart rate were observed. We observed a moderate rise in the dPdt max (from $1361 \mathrm{mmHg} / \mathrm{s}$ to $1423 \mathrm{mmHg} / \mathrm{s}$ ), as well as a significant rise in peak ejection rate (PER) from $280(\mathrm{ml} / \mathrm{s})$ before the shunt to $446(\mathrm{ml} / \mathrm{s})$ immediately afterward. All these dramatic changes were reversible at this point in time, by closure of the shunt. These results suggest that the wide changes in the left ventricular model are within the efficient part on Starling's curve.

Another advantage of the shunt model is that it is possible to evaluate the patency of the shunt by palpating the blood thrill in the neck. The shunt's location facilitates access to ultrasonic measures for a more precise evaluation, as well as temporary closure, obtained by applying manual pressure at the animal's neck.

Our study ended 8 weeks after the creation of the A-V shunt, leaving unanswered the question of possible heart failure development in this model after a longer follow up period.

In conclusion, the carotid jugular $\mathrm{A}-\mathrm{V}$ shunt in goats causes significant left heart dilatation without signs of heart failure, and is reproducible with low animal mortality. 


\section{REFERENCES}

I Cohn JN. Current therapy of failing heart. Circulation 1988; 78:1099-1107.

2 Swedberg $\mathrm{K}$. Reduction in mortality by pharmacological therapy in congestive heart failure. Circulation 1993; 87 (suppl. IV): IV-126-IV-129.

3 Muders F, Friedrich E, Luchner $\Lambda$, Pfeifer M, Ickenstein G. Hemodynamic changes and neurohemoral regulation during development of congestive heart failure in a model of epinephrin-induced cardionyopathy in conscious rabbits. J Card Fail 1999;5(2): 109-116.

4 Winslow RL, Rice J, Jafri S, Marban E, O'Rourke B. Mechanisms of altered excitationcontraction coupling in canine tachycardia-induced heart failure, II: model studies. Circ Res 1999; 19;84(5):571-586.

5 Tofovic SP, Kusake H, Rominski B, Jackson EK. Caffeine increases renal renin secretion in a rat model of genetic heart failure. J Cardiovase Pharmacol 1999;33(3):440-450.

6 Nishio R, Matsumori A, Shioi T, Wang W, Yamada T, Ono K, Sasayama S, Denopamine, a beta 1 - adrenergic agonist, prolongs survival in a murine model of congestive heart failure induced by viral myocarditis: suppression of tumor necrosis factor-alpha production in the heart. J Am Coll Cardiol 1998;32(3):808-815.

7 Genao A, Seth K, Schmidt U, Carles M, Gwathmey JK. Dilated cardiomyopathy in turkeys: an animal model for the study of human heart failure. Lab Anim Sci 1996;46(4):399-404.

8 Sabbah HN, Stein PD, Kono T, Gheorghiade M, Levine TB, Jafri S, Hawkins IT, Goldstein S. A canine model of chronic heart failure produced by multiple sequential coronary nicroembolizations. Am J Physiol 1991;260(4 Pt 2):H1379-1384.

9 Desjardins S, Mueller RW, Cauchy MJ. $\Lambda$ pressure overload model of congestive heart failure in rats. Cardiovasc Res 1988;22(10):696-702.

10 Flaim SF, Minteer WJ, Nellis SH, Clark DP. Chronic arteriovenous shunt: evaluation of a model for heart failure in rat. Am J Physiol 1979;236(5):H698-704.

11 Vasan RS, Larson MG, Benjamin EJ, Evans JC, Levy D. Left ventricular dilatation and the risk of congestive heart failure in people without myocardial infarction. Engl J Med 1997;336(19):1350-1355.

12 Porter CB, Walsh RA, Badke FR, O'Rourke RA. Differential effects of diltiazem and nitroprusside on left ventricular function in experimental chronic volume overload. Circulation 1983;68:685-692.

13 Wegner M, Hirth-Dietrich C, Stasch JP. Role of neutral endopeptidase 24.11 in AV fistular rat model of heart failure. Cardiovasc Res 1996;31(6):891-898. 
Chapter

\section{Peri-operative hemodynamic and geometrical changes of the left ventricle during cardiomyoplasty in goats with dilated left ventricle}

Gil Bolotin, Roberto Lorusso, Jan J Schreuder, Hans Kaulbach, Gideon Uretzky, Frederik van der Veen

Presented at the Mediterranean Association of Cardiology and Cardiac Surgery, 9th Annual Meeting, Tel Aviv, Israel, October 1996. Isr J Med Sci, 1996;32:911. 


\begin{abstract}
Objective. Clinical data suggest temporary acute deterioration following cardiomyoplasty. The purpose of this study was to monitor the acute hemodynamic effects of cardiomyoplasty in a goat model of dilated left ventricle, using conductance catheters (pressure volume loops) and cardiac output measurements.

Methods. Eight female goats underwent acute cardiomyoplasty 8-12 weeks after inducing left ventricular (LV) dilatation by carotid jugular artero-venous (AV) shunt. The cardiomyoplasty procedure was monitored using a SwanGanz catheter for cardiac output measurements and a 12-electrode (dualfield) conductance catheter to LV pressure volume loops.

Results. After wrapping the heart with the latissimus dorsi muscle, there was a significant reduction in both cardiac output and LV end-diastolic volume at 10 minutes. Partial recovery was observed 45 minutes later.

Conclusion. A decrease in both cardiac output and LV end-diastolic volume was observed following myocardial wrapping. This may explain some of the perioperative and postoperative morbidity and mortality observed following cardiomyoplasty.
\end{abstract}

Keywords. Cardiomyoplasty, Conductance catheters, Heart failure model. 


\section{INTRODUCTION}

The acute peri-operative hemodynamic and geometrical changes after cardiomyoplasty are not fully understood (1). Corin and coworkers demonstrated early postoperative impairment of left ventricular (LV) diastolic function in a healthy dog model following cardiomyoplasty (2). In contrast, Lazzara and associates report little effect on LV end-diastolic volume in healthy dogs submitted to cardiomyoplasty (3). The aim of this work was to evaluate the acute hemodynamic and geometrical changes of the left ventricle during and immediately after the cardiomyoplasty procedure. The arterovenous (AV) shunt was chosen to induce heart dilatation in a goat model in order to simulate the end-stage dilated cardiomyopathy.

\section{METHODS}

\subsection{The carotid jugular AV shunt model}

Eight female goats, weighing 51 to $80 \mathrm{~kg}$, were used for the experimentation. All experiments were performed in accordance with the "Guide for the Care and Use of Laboratory Animals"(4). In the AV shunt surgical procedure, general anesthesia was induced by thiopental sodium (Pentothal, Abbott S.P.A., Italy) with $15 \mathrm{mg}$ per $\mathrm{kg}$ body weight iv and, after endotracheal intubation, maintained with oxygen/nitrous oxide (1:2) and 1.5\% Fluothane (Halothane, Zeneca L.td., Macclesfield, Cheshire, UK). During the experiments, the lungs were ventilated with a positive pressure respirator (Harvard, Apparatus Inc., South Natick, Massachusetts) and body temperature was maintained constant with a heating mattress. A single dose of $10,000 \mathrm{U}$ i.v. heparin was administered. Through a left cervical incision, the left jugular vein and the left carotid artery were exposed for a length of approximately $5 \mathrm{~cm}$ and ligated distally. After cross-clamping of the left carotid artery, an end-to-side anastomosis of about $1 \mathrm{~cm}$ in diameter between the free end of the vein and the side of the artery was performed, using 6-0 polypropylene (Ethicon, Edinburgh, Scotland), non-absorbable running sutures. Clamps were removed, and the patency of the fistula was confirmed by the pulsating filling of the jugular vein. The entire surgical procedure was completed in approximately 120 minutes.

Baseline measurements included cardiac output before and after performing the AV shunt ( 8 goats). Left heart pressure-volume loops were attained by the conductance catheter method before and after performing the $\mathrm{AV}$ shunt ( 3 goats). Baseline LV dimensions and wall thickness were measured by means of transthoracic echocardiography. Follow-up studies were 
performed 2 and 4 weeks after establishing the AV shunt, to examine ECCG, LV dimensions (short and long axis), and LV thickness (free wall and septum) by means of transthoracic echocardiography.

\subsection{The cardiomyoplasty procedure}

The cardiomyoplasty procedure was performed 8-10 weeks after the AV shunt procedure. General anesthesia was induced using the same drugs and equipment as in the AV shunt procedure. A left-sided, midaxillary incision was performed and all collateral blood vessels to the distal part of the latissimus dorsi (LD) muscle were coagulated. All attachments of the muscle were disconnected, taking care to preserve the axillary pedicle, in order to keep the thoracodorsal artery, vein, and nerve intact. Two intramuscular electrodes (Telectronics IML - 04B) were implanted in the upper part of the LD muscle flap, perpendicular to the main branches of the thoracodorsal nerve, as described by Chachques and coworkers (5). To assure proper positioning, threshold $(0.3-0.6 \mathrm{v})$, total recruitment $(1.0-2.5 \mathrm{v})$, and impedance $(220-300$ $\mathrm{ohm}$ ) values of the stimulation electrodes were measured by connecting these electrodes to a pacing system analyzer (Telectronics Pacing System PSA 2401). A $5 \mathrm{~cm}$ segment of the anterior portion of the third rib, including the periosteum, was then resected to allow transposition of the LD muscle flap into the thorax. The muscle was inserted into the chest cavity, its tendon was cut and sutured to the periosteum of the fourth rib, while closing the thoracic window. The thoracic cavity was opened at the fourth left intercostal space and the pericardium was opened. A sensing, pacing electrode (Telectronics 033 - S72) was implanted in the left ventricular wall and sensing (4.5-16.4 v) and impedance (210-290 ohm) measurements were recorded. The left LD muscle flap was than wrapped in a counterclockwise fashion around both ventricles. The muscle was first positioned around the right ventricle, and fixed near the atrioventricular groove at the base of the heart with interrupted sutures, after which the remaining part of the muscle was wrapped around the left ventricle. This distal portion was then sutured to the proximal part of the muscle.

\subsection{Monitoring}

To monitor the surgical procedure, cardiac output and pressure volume loops were taken before and after each step of the surgical procedure, including the introduction of the muscle into the chest cavity through the chest window (just cardiac output), and the wrapping of the heart. The same measurements were taken 10 and 45 minutes after accomplishing the heart wrapping. These 
measurements included cardiac output and central venous pressure obtained by means of a Swan-Ganz catheter, LV pressure and volume values obtained by a dual-micromanometer transducer conductance catheter (7F, Sentron, The Netherlands), and aortic pressure measured with a Sentron catheter $(7 \mathrm{~F}$, Sentron, The Netherlands).

For right heart catheterization, a skin incision was made laterally to the right external jugular vein. Through a $2 \mathrm{~mm}$ longitudinal phlebotomy in the external jugular vein, a Swan-Ganz thermodilution catheter was introduced into the pulmonary artery. The catheter was secured by purse-string sutures in the external jugular vein. To obtain reliable estimates of cardiac output by thermodilution, a computer-controlled injection system was used. Cardiac output was determined by injections of $10 \mathrm{ml}$ ice-cold glucose $5 \%$ using a cardiac output computer (COM-2, Baxter). Through the left femoral vein and under fluoroscopic guidance, the balloon of an occluder catheter was placed into the upper part of the inferior caval vein.

For left heart catheterization, a 12-electrode (dual-field) conductance catheter (7F, Sentron) was placed, via the left femoral artery and under fluoroscopic guidance, along the long axis of the LV cavity. An aortic pressure catheter was placed via the right femoral artery.

The position of the conductance catheter was verified by inspection of the segmental conductance signals. The appropriate location of the conductance catheter was assumed if the signals from at least the four most distal segments displayed a typical phasic LV volume tracing. If the most proximal segment reflected typical phasic atrial volume changes, this segment was excluded from the calculation of the total ventricular volume. Correct positioning of the conductance catheter was further facilitated by on-line display of the LV contour, derived from the four or five segmental conductance signals.

The conductance catheter determines ventricle volume on-line by measuring time-varying electrical conductance of segments of intraventricular blood. Total ventricular volume was calculated from these measurements using formulae that have been described previously (6). The pigtail catheter was especially designed, equipped with $1 \mathrm{~mm}$-long electrodes placed $10 \mathrm{~mm}$ apart from each other. An alternating current of $0.03 \mathrm{~mA}$ at $20 \mathrm{kHz}$ was passed through the first two electrodes, and voltages were measured between the four or five adjacent electrode pairs ( 2 to 3 through 6 to 7 ), from which five conductances were calculated. Electrodes 9 and 10 were not used. A Leycom Sigma-5DF signal conditioner-processor (CardioDynamics) provided the current source and processed the segmental conductances, producing an online display of the $\mathrm{LV}$ contour as well as a continuous and instantaneous volume signal. The volume signal of the ventricle was combined with the LV 
pressure signal on an X-Y oscilloscope, in order to continuousiy display pressure-volume loops in real time.

The correction of the volume signal for the parallel conductance caused by tissues surrounding the ventricular cavities was done by injecting a bolus of $7.5 \mathrm{ml}$ of hypertonic $\mathrm{NaCl}(9 \%)$ into the central venous compartment via the Swan-Ganz catheter. As the bolus mixes with the fluid in the ventricular cavity, its conductivity increases, causing the overall conductance signal to increase, while the parallel component remains constant. End-systolic overall conductance was then plotted as a function of end-diastolic, overall conductance during the mixing of the bolus; the parallel conductance was equal to the intersection point between the regression line of these values and the line of identity.

Statistical analysis was performed with Student's test for paired variants. Values are presented as mean \pm SD. Significance was assumed at $\mathrm{P}<0.05$.

\section{RESULTS}

\subsection{The carotid jugular AV shunt model}

The carotid jugular AV shunt induced both immediate and long-term hemodynamic effects. The immediate results of the shunt consisted of a marked increase in the cardiac output to $7.9 \pm 1.7 \mathrm{l} / \mathrm{m}$, only a few minutes after the AV shunt was opened, compared to the average baseline cardiac output of $4.9 \pm 1.4$ $1 / \mathrm{m}$.

The long-term results of the shunt were monitored by echocardiography and cardiac output measurements. As compared to baseline measurements, a consistent significant increase in the LV end-diastolic diameter was observed 2,4 , and 8 weeks following the AV shunt procedure. Eight weeks after the shunt, a further increase in cardiac output was observed $(9.9 \pm 2.7 \mathrm{l} / \mathrm{m})$.

\subsection{The Cardiomyoplasty procedure}

During the cardiomyoplasty procedure, cardiac output decreased slightly but significantly after the LD muscle was introduced into the chest (6\%); however, it decreased by an additional $18 \%$ immediately after the wrapping procedure, and was partially improved 45 minutes later. Average cardiac output results measured during the cardiomyoplasty procedure are presented in Figure 1.

The wrapping procedure was monitored with conductance catheters showing the pressure volume loops before and after the wrapping. After the wrapping, a significant reduction was observed in the mean LV end-diastolic 

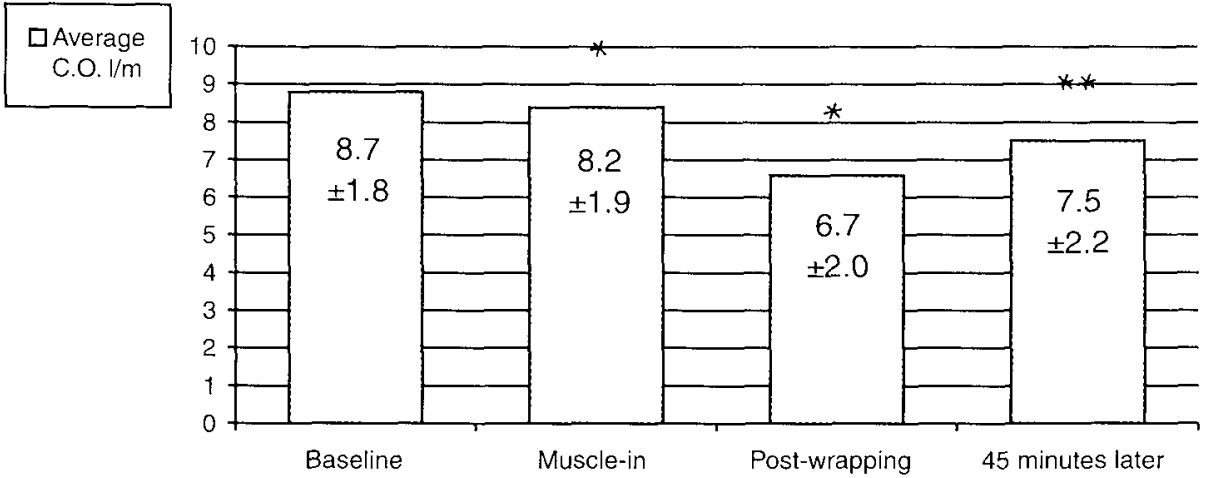

Figure 1. Average cardiac output measured by Swan-Ganz catheters at different stages of the cardiomyoplasty procedure. Baseline - following anesthesia. Muscle in - after introducing the LD muscle into the thorax. 10 minutes post wrapping - after the LD muscle was attached to the ventricles. 45 minutes post wrapping - after a recovery period of 45 minutes.

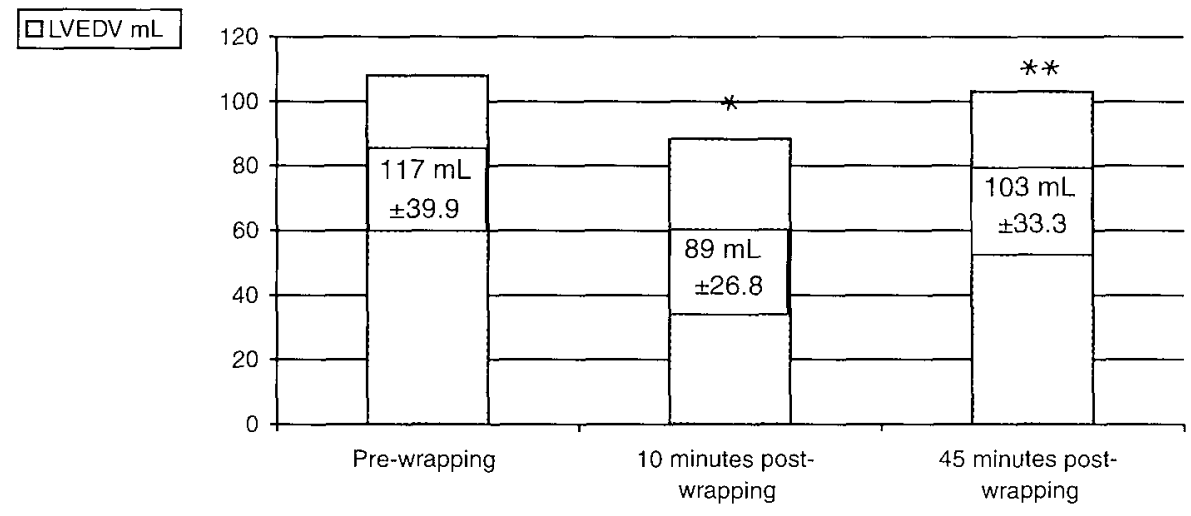

Figure 2. The average end-diastolic left ventricular volume measured with conductance catheters before, 10 minutes after, and 45 minutes after the wrapping.

volume; 45 minutes later a partial recovery was noted (Figure 2). An example of pressure-volume loops before, immediately after, and 45 minutes after the wrapping is presented in Figure 3.

Heart rate, LV end-systolic volume, stroke volume and ejection fraction before and after the wrapping are presented in Table 1. 


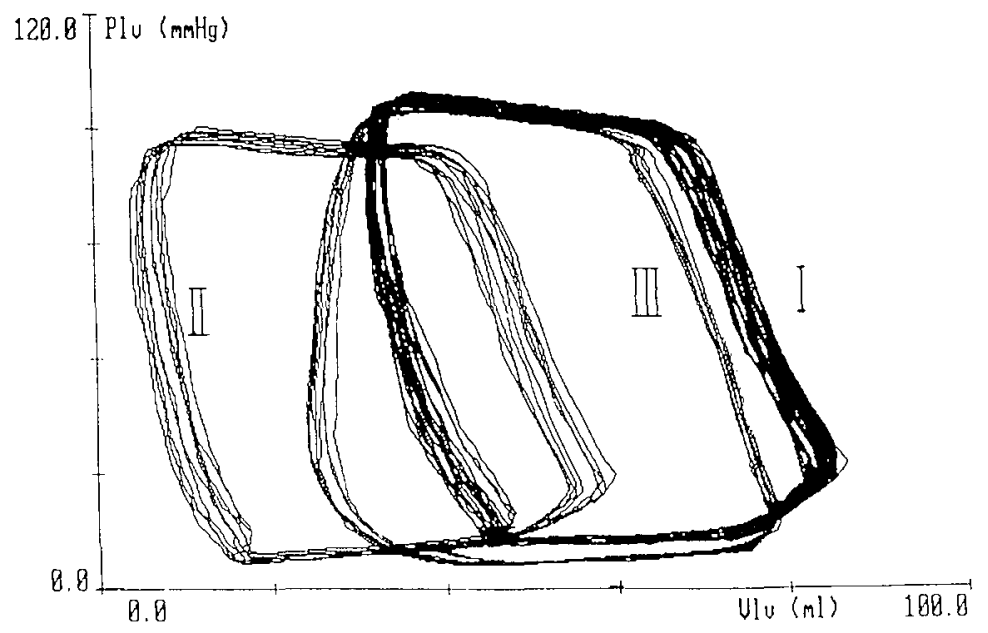

Figure 3. I - Pressure volume loops before wrapping. II - Pressure volume loops immediately after wrapping. III - Pressure volume loops 45 minutes after wrapping.

Table 1. Hemodynamic data before, 10, and at 45 minutes after the wrapping of the latissimus dorsi muscle around the heart.

\begin{tabular}{|c|c|c|c|c|c|}
\hline & $\begin{array}{l}\mathrm{HR} \\
\text { (beat/min) }\end{array}$ & $\begin{array}{l}\text { LVEDV } \\
(\mathrm{ml})\end{array}$ & $\begin{array}{l}\text { LVESV } \\
(\mathrm{ml})\end{array}$ & $\begin{array}{l}\text { SV } \\
(\mathrm{ml})\end{array}$ & $\begin{array}{l}\mathrm{EF} \\
(\%)\end{array}$ \\
\hline Pre-wrapping & $104 \pm 7$ & $117 \pm 40$ & $36 \pm 17$ & $81 \pm 29$ & $68 \pm 6$ \\
\hline 10 min post wrapping & $96 \pm 13$ & $89 \pm 27$ & $20 \pm 12$ & $69 \pm 15$ & $78 \pm 7$ \\
\hline P value & 0.04 & 0.03 & 0.003 & NS & 0.006 \\
\hline 45 min post wrapping & $97 \pm 16$ & $103 \pm 33$ & $34 \pm 17$ & $69 \pm 29$ & $66 \pm 8$ \\
\hline $\begin{array}{l}\text { P value as compare to } 10 \\
\text { minutes post wrapping }\end{array}$ & 0.05 & 0.02 & 0.05 & NS & 0.05 \\
\hline $\begin{array}{l}\text { P value as compare to pre } \\
\text { wrapping }\end{array}$ & NS & 0.05 & NS & NS & NS \\
\hline
\end{tabular}

HR - Heart rate. LVEDV - Left ventricular end diastolic volume. LVESV - Left ventricular end systolic volume. SV - Stroke volume. EF - Ejection fraction. NS indicates not significant $(\mathrm{p}>0.05)$. 


\section{DISCUSSION}

\subsection{The carotid jugular AV shunt model}

The AV shunt model is capable of producing immediate, stable, and long-term high cardiac output (overload) LV dilatation. None of the eight goats demonstrated clinical signs of pulmonary congestion. Our findings are in contrast with the high mortality rates $(77 \%)$ and signs of pulmonary congestion that commonly occur within 24 hours of performing the AV shunt in small animals, as reported by Flaim and coworkers (7). This discrepancy is probably due to the relatively limited AV shunt (approximately $10 \mathrm{~mm}$ ) used in our study in goats weighing 51 to $80 \mathrm{~kg}$. Moreover, in the present study, the animals presented neither clinical nor hemodynamic signs of heart failure, such as tachycardia or an increase in LVEDP, as was observed with inferior vena cava aortic AV shunt applied in mongrel dogs (8). The successful compensatory mechanism in the goat carotid jugular AV shunt model is the reason for the lack of signs of renal failure, such as were demonstrated in a rat aorto-caval fistula by Wegner and coworkers (9). Therefore, the model described should be considered a compensatory overload dilatation model rather than a heart failure model.

\subsection{The cardiomyoplasty procedure}

The wrapping procedure monitored in our study induced major reduction in both cardiac output and LV volume followed by partial recovery 45 minutes later. These results are consistent with the clinical reports of acute deterioration in the immediate postoperative period for which Carpentier and Mesana proposed extensive use of IABP and inotropic support before and after the operation $(10,11)$. In an attempt to deal with the acute hemodynamic changes, the cardiomyoplasty procedure has undergone major technical changes since the first clinical experience (12). Two major changes in the surgical technique were the "non-cardiac suture" technique (13) and the "flap sliding maneuver" (10). These technical improvements were one of the main factors that contributed to a reduction of up to $12 \%$ in operative mortality (14). Efforts have been made to identify the optimal length of the LD muscle (as compared to its original resting length) necessary for more effective mechanical performance (15) as well as the best LD muscle orientation for dynamic cardiomyoplasty (16). Gealow and coworkers demonstrated the adaptive changes of skeletal muscle conformational after the wrapping procedure, suggesting the lack of need to stretch the LD muscle to reproduce in situ muscle tension and length (17). Pericardial "suspenders," described by Lorusso and Alfieri and introduced 
during right cardiomyoplasty, not only enabled this procedure to be carried out using the "flap sliding maneuver" but, perhaps more importantly, enabled the wrapping tightness to be controlled (18). Under direct transesophageal echocardiographic observation, adjustment of the wrapping tightness was achieved by pulling the pericardial strips connected to the LD muscle flap.

Monitoring the cardiomyoplasty procedure under direct transesophageal echocardiographic observation also gave important data in real time, enhancing control of certain steps in the surgical procedure. However, exact quantitative hemodynamic data concerning the effects of the wrapping are still missing in the literature. In this study, efforts were made to monitor the hemodynamic effects of the wrapping procedure. Monitoring of both cardiac output and left ventricular volumes, before, during, and after the surgical procedure, provides new data about the immediate physiologic changes.

Both cardiac output and LV end-diastolic volume decreased slightly, yet statistically significantly, after introducing the LD muscle into the thorax through the intercostal window and prior to the thoracotomy. Our data demonstrated that this step of the surgical procedure exerts dangerous hemodynamic effects, most probably due to the slight elevation in left chest internal pressure and direct heart compression. However, it is important to note that the harmful influence that this stage usually has on respiratory function was not monitored in this study. The wrapping, in contrast, had a major effect on both cardiac output and the LV end-diastolic volume. Immediately after the wrapping there was an $18 \%$ reduction in cardiac output and a total reduction of $22 \%$, including the reduction due to the introduction of the LD muscle into the chest. These extreme results can be explained by analyzing the data recorded by the conductance catheter. The $24 \%$ reduction of the LV end-diastolic volume measured 10 minutes after wrapping indicates a major change in left heart hemodynamics due to the external pressure of the LD muscle around the heart. The slight reduction in stroke volume represents the partial compensatory mechanism, with the rise in $\mathrm{LV}$ ejection fraction due to the reduction in LV end-systolic volume. The slower heart rate after the wrapping emphasizes the non-physiologic hemodynamic situation ten minutes after the wrapping. The major reduction of LV end-diastolic volume measured ten minutes after wrapping is in contrast with the findings of other investigators, who showed that some reduction in LV diastolic function was demonstrated but without reduction in volumes $(2,3,18)$. The explanation for this discrepancy may be the combination of the LV dilatation model and the sensitive methods of hemodynamic monitoring used in this study. This assumption is supported by the clinical data published by Barbier and coworkers, demonstrating acute modification of left and right ventricular geometry as monitored by echocardiography immediately after cardiomyoplasty (19). 
These findings underscore some potential risks of cardiomyoplasty and might explain some of the perioperative mortality associated with the procedure, especially in patients with markedly dilated heart and severely impaired IV function who might lack the reserve to overcome this dangerous phase of the cardiomyoplasty procedure (20).

The partial recovery within 45 minutes in both cardiac output (86\%) and LV end-diastolic volume (88\%) seems to be a relatively quick recovery, but, in our study, it might be related to the compensated, not the failed, dilated heart model. The AV shunt probably moved the LV to a new balanced position on the Frank-Starling curve. Based on clinical information, one can estimate that this recovery process might take days in heart failure patients (21). In conclusion, our data suggest that in the acute post cardiomyoplasty phase the heart attains a new tension/length balance with a smaller LV end-diastolic volume before the stimulation protocol is commenced. This may explain the clinical improvements sometimes seen in these patients at that early postoperative stage. 


\section{REFERENCES}

l Blanc P, Girard C, Vedrinne C, Mikaeloff P, Estanove S. Latissimus dorsi cardiomyoplasty. Perioperative management and postoperative evolution. Chest 1993 Jan; 103(1):214-220.

2 Corin WJ, George DT, Sink JD, Santamore WP. Dynamic cardiomyoplasty acutely impairs left ventricular diastolic function. J Thorac Cardiovasc Surg 1992;104: 1662-1671.

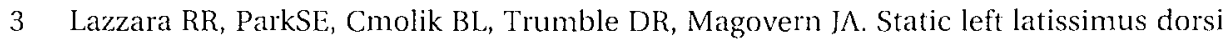
cardiomyoplasty: effect on left ventricular function. J Heart lung Transplant 1993;12:1024-1080.

4 National Academy of Sciences: Guide for the Care and Use of Laboratory Animals. DHHS publication No. NIH 85-23, revised 1985.

5 Chachques JC, Grandjean PA, Carpentier $\Lambda$, Latissimus dorsi dynamic cardiomyoplasty. Ann Thorac Surg, 1989; 47: 600-604.

6 Schreuder JJ, Biervliet JD, Van der Velde ET, Ten Have K, Van Dijk AD, Meyne NG and Baan J. Systolic and diastolic pressure-volume relationships during cardiac surgery. J Cardiothorac Vasc Anesth, 1991; 5: 539-545.

7 Flaim SF, Minteer WI, Nellis SH, Clark DP. Chronic a-v shunt: evaluation of a model for heart failure in rat. Am J Physiol 1979;236(5):H698-704.

8 Porter $\mathrm{CB}$, Walsh $\mathrm{R} \Lambda$, Badke FR, O'Rourke RA. Differential effects of diltiazem and nitroprusside on left ventricular function in experimental chronic volume overload. Circulation 1983;68:685-692.

9 Wegner M, Hirth-Dietrich C, Stasch JP. Role of neutral endopeptidase 24.11 in AV fistular rat model of heart failure. Cardiovasc Res 1996;31 (6):891-898.

10 Carpentier A, Chachques JC, Acar C, Relland I, Mihaileanu S, Bensasson D, Kieffer JP, Giubourt P,Tournay D, Roussin I, Grandjean PA. Dynamic cardiomyoplasty at seven years. J Thorac Cardiovasc Surg 1993;106:42-53.

11 Mesana TG, Bauer S, Caus T, Pomane C, Mouly A, Monties JR. Circulatory assist techniques after cardiomyoplasty: determinants for clinical outcome and later consequences. ASAIO J 1995;41:M469-M472.

12 Carpentier A, Chachques JC. Myocardial substitution with a stimulated skeletal muscle: first successful clinical case. Lancet 1985; 1:1267.

13 Carpentier A, Chachques JC. Surgical technique in: Carpentier A, Chachques JC, Grandjean PA (eds), Cardiomyoplasty. New York: Futura, 1991: 105-122.

14 Carpentier A, Chachques JC and Grandjean PA. Mechanical circulatory support, Dynamic cardiomyoplasty at nine years, Book. 277-284.

15 Soltero ER, Michael LH, Glaeser DH, Hartley CJ, Earle NR, Lawrie GM. New configuration of double cardiomyoplasty based on studies of length-tenth properties of the latissimus dorsi muscle. J Thorac Cardiovasc Surg 1993; 106: 842-849.

16 Kao RL, Kao RL, Christlieb IY, Magovern GJ, Park SB, Magovern GJ Jr. The importance of skeletal muscle fiber orientation for dynamic cardiomyoplasty. J Thorac Cardiovasc Surg 1990; 99: 134-140.

17 Gealow KK. Solien EE. Bianco RW. Chiu RC. Shumway SJ. Conformational adaptation of muscle: implications in cardiomyoplasty and skeletal muscle ventricles. Ann Thorac Surg. 1993; 56(3): 520-526.

18 Lorusso R and Alfiery O. Pericardial "suspenders" to enhance right latissimus dorsi 
19 Polidori DJ. Lankford EB, Plappert T. Ferrari VA. Sutton MS. Kass DA. Acker MA. Acute systolic and diastolic indices of left ventricular function after cardiomyoplasty in chronic model of heart failure. ASAIO J 1995;41 (3):M484-489.

20 Barbier P. Gerometta P. Tamborini G. Biglioli P. Sisillo E. Guazzi MD. Acute effects of dynamic cardiomyoplasty on ventricular geometry and left ventricular filling detected by transesophageal doppler echocardiography. Am J Cardiol 1996; 77(9); 783-787.

21 Grandjean PA, Austin L, Chan S, Terpstra B, Bourgeois IM. Dynamic cardiomyoplasty: Clinical follow-up results. J Cardiac Surg 1991; 6(suppl): 80-88.

22 Delahaye $F$, Jegaden $O$, Montagna P, Desseigne P, Blank P, Vedrinne C, Touboul P. Latissimus dorsi cardiomyoplasty in severe congestive heart failure: the Lyon experience. J Cardiac Surg 1991; (suppl.):106-112. 



\section{Early effects of cardiomyoplasty in a goat model of ventricular dilatation}

Gil Bolotin, Jan J. Schreuder, Hans G. Kaulbach, Roberto Lorusso, Gideon Uretzky, Frederik van der Veen

Presented at Cardiostim 10th International Congress, Nice, France, June 1996. The European Journal of Cardiac Pacing and Electro- physiology, 1996; 6 (supp): 153. 


\section{ABSTRACT}

Objective. Cardiomyoplasty induces a remodeling effect without producing a clear systolic augmentation. We hypothesized that this deficiency is mainly due to the resultant reduction in heart dilatation.

Methods. A chronic carotid jugular (AV) shunt model in goats was used to induce dilatation of the ventricles. Subsequently, acute cardiomyoplasty was performed and the assisted cardiac beats were compared to unassisted cardiac beats using the conductance catheter method to generate PressureVolume loops.

Results. Due to the AV-shunt, left ventricular end-diastolic diameter increased by $26 \%$, from $42 \pm 4 \mathrm{~mm}$ to $53 \pm 4 \mathrm{~mm}$ ( $\mathrm{p}<0.05)$ during an 8 week follow-up period. Immediately after the subsequent cardiomyoplasty procedure, a significant improvement in stroke volume $(117 \pm 48 \mathrm{ml}$ Vs $87 \pm 38 \mathrm{ml})$ and a reduction in left ventricular end-systolic volume were observed when comparing assisted beats with unassisted beats.

Conclusion. Gradual reduction of LV size after cardiomyoplasty may explain the absence of systolic augmentation when using clinical stimulation settings. 


\section{INTRODUCTION}

Clinical results after cardiomyoplasty (CMP) show functional class improvement and amelioration of quality of life (1). The true role of systolic assist in obtaining these results is still unclear, as clinical improvement has been documented both with and without marked changes in cardiac output $(2,3,4)$. However, this discrepancy might be explained by the findings of Schreuder (5) and Kass (6), which demonstrate gradual remodeling of the left ventricle (LV) after a cardiomyoplasty procedure. In these patients, the dilated LV was reduced to about $50 \%$ of the preoperative volume, while stimulation of the latissimus dorsi (LD) muscle had only minor systolic hemodynamic effects when using the clinical stimulation protocol (5). Moreover, using a goat model with an A-V shunt, Kaulbach et al. demonstrated a significant reduction of the $\mathrm{LV}$ volumes at the end of the latissimus dorsi conditioning period (eight weeks); yet again, systolic augmentation at that time was not significant (7)

Therefore, we hypothesized that a significant hemodynamic effect can only be generated as long as the LV dilatation exists. This dilatation characterizes the clinical situation immediately following CMP surgery, at which time the LD muscle has not yet been transformed into a slow, fatigue resistant muscle. This hypothesis was examined immediately after CMP in an animal model with a left ventricle dilated through use of a chronic AV-shunt.

\section{METHODS}

The experiments were performed in eight female goats, weighing 51 to $80 \mathrm{~kg}$, in accordance with the Guide for the Care and Use of Laboratory Animals published by the National Institutes of Health (NIH publication 86-23, revised 1985).

Dilated cardiomyopathy was induced by a left carotid jugular AV shunt. The animals' condition was followed 8 to 10 weeks using echocardiography, after which acute cardiomyoplasty was performed. Monitoring with pressurevolume loops using the conductance catheter technique was done during the $\mathrm{A}-\mathrm{V}$ shunt procedure and at the final acute cardiomyoplasty experiment (8-10 weeks later).

\section{AV shunt procedure}

General anesthesia was induced by thiopental sodium (pentotal Abbot s.p.a. Italy), administered intravenously at $15 \mathrm{mg}$ per $\mathrm{kg}$ body weight, and main- 
tained after endotracheal intubation with oxygen/nitrous oxide (1:2) and $1.5 \%$ Fluothane (Halothane, Zeneca Ltd., Macclesfield, Cheshire, UK).

During the experiments, the lungs were ventilated with a positive pressure respirator (Harvard, Apparatus Inc., South Natick, Massachusetts) and body temperature was kept constant with a heating mattress. A single dose of 10.000 IU heparin i.v. was administered. Through a cervical incision, the left jugular vein and the left carotid artery were mobilized over a length of approximately $5 \mathrm{~cm}$ and ligated distally. After cross-clamping of the left carotid artery, an end-to-side anastomosis of about $1 \mathrm{~cm}$ in diameter was performed between the free end of the vein and the side of the artery, using 6-0 polypropylene non-absorbable rumning sutures (Ethicon). Clamps were removed and the patency of the fistula was confirmed visually by the pulsatile filling of the jugular vein.

\section{Baseline measurements}

Cardiac output was measured before and after performing the A-V shunt, using a Swan-Ganz thermodilution catheter $(7 \mathrm{~F})$. Left heart pressure-volume loops were obtained before and after performing the AV shunt, using the conductance catheter method. Baseline left ventricular dimensions were measured by transthoracic echocardiography. LV-dimensions were measured by echocardiography 2,4 , and 8 weeks after establishing the AV shunt.

The final experiment, performed 8-10 weeks after the AV shunt procedure once left ventricular end-diastolic diameter (LVEDD) had increased significantly, included left and right heart catheterization followed by the cardiomyoplasty procedure and a subsequent immediate stimulation protocol. General anesthesia was induced as described for the AV shunt surgical procedure.

\section{Heart catheterization}

A dual-micromanometer transducer conductance catheter $7 \mathrm{~F}$, Sentron, The Netherlands) was used to determine the volume of the ventricle on-line, by measuring the time-varying electrical conductance of the segments of intraventricular blood (8). Its position, along the long axis of the left ventricular cavity via the left femoral artery, was set under fluoroscopic guidance and verified by inspection of the segmental conductance signals: a correct position was assumed if the signals of at least the four most distal segments displayed a typical phasic LV volume tracing. A Leycom Sigma-5DF signal conditioner-processor (CardioDynamics, Zoetermeer, The Netherlands) provided the current source and processed the segmental conductance, 
producing an on-line display of the LV contours, as well as continuous and instantaneous volume signals. The volume signals were combined with the pressure signals on an $X-Y$ oscilloscope to provide an instantaneous and uninterrupted display of pressure-volume loops.

Readings of the volume signal were corrected to account for the parallel conductance caused by tissues surrounding the ventricular cavities. A bolus of $7.5 \mathrm{ml}$ of hypertonic $\mathrm{NaCl}(9 \%)$ was injected into the central venous compartment via the Swan-Ganz catheter. As the bolus mixed with the fluid in the ventricular cavity, its conductivity increased, causing the overall conductance signal to increase while the parallel component remained constant. Endsystolic overall conductance was then plotted as a function of end-diastolic overall conductance during the mixing of the bolus; the conductance was equal to the intersecpoint between the regression line of these values and the line of identity.

\section{Cardiomyoplasty procedure}

A left-sided, midaxillary incision was performed, and all collateral blood vessels to the distal part of the latissimus dorsi muscle were coagulated. All attachments of the muscle except the axillary pedicle were disconnected to keep the thoracodorsal artery, vein, and nerve intact. Two intramuscular electrodes (Telectronics IML - 04B) were implanted in the upper part of the latissimus dorsi muscle flap, perpendicular to the main branches of the thoracodorsal nerve, as described by Chachques and coworkers (9). To assure proper positioning, threshold (0.3-0.6 V), total recruitment (1.0-2.5 V), and impedance (220-300 ohm) of the stimulation electrodes were measured using a pacing system analyzer (Telectronics Pacing System PSA 2401). A $5 \mathrm{~cm}$ segment of the anterior portion of the third rib, including the periosteum, was then resected to allow transposition of the latissimus dorsi muscle into the thorax. The muscle was inserted into the chest cavity, its tendon was cut and sutured to the periosteum of the fourth rib, while closing the thoracal window. The thoracic cavity was opened at the fourth left intercostal space, and the pericardium was opened. A sensing/pacing electrode (Telectronics 033 - S72) was implanted in the right ventricular wall, and sensing and impedance were verified to assure proper positioning. The left latissimus dorsi muscle flap was wrapped in a counterclockwise fashion around both ventricles. The muscle was first positioned around the right ventricle and fixed with interrupted sutures near the atrioventricular groove at the base of the heart. Then the remaining part of the muscle was wrapped around the left ventricle. The distal portion was sutured to the proximal part of the muscle. 
The stimulation protocol was performed about 45 minutes after accomplishing the wrapping, and the hemodynamic effects were recorded by IV conductance catheters presenting real time, beat-to-beat pressure-volume loops.

The first part of the stimulation protocol included bursts of six pulses (5 volt, $650 \mu \mathrm{s}$-pulse width and $160 \mathrm{~ms}$ burst duration) at varying delays. The measurements were obtained during stimulation with varying delays between the Qrs complex and the beginning of the stimulation burst $(25,50,75,100$, 125 and 150 milliseconds). The second measurement was done at an amplitude of 10 volts instead of 5 volts, using the best delay, as determined during the former experiment, according to the shape of the pressure volume loops.

The third part of the study protocol included LD muscle stimulation with only one pulse every four spontaneous heart beats for 10 minutes. Then, the stimulator was blocked electrically to demonstrate reversibility, if any, for another period of 10 minutes.

Statistical analysis was performed with Student's test for paired variate. Values are presented as mean \pm SD. Significance was assumed at $\mathrm{P}<0.05$.

\section{RESULTS}

\section{AV-shunt model}

The carotid-jugular AV-shunt induced immediate and long-term hemodynamic changes. The immediate effect of the shunt was an abrupt rise in cardiac output to $7.9 \pm 1.9 \mathrm{l} / \mathrm{min}$, within 30 minutes after opening of the shunt, as compared to the baseline cardiac output of $4.9 \pm 1.3 \mathrm{l} / \mathrm{min}$. Eight weeks after the shunt procedure, a further increase in cardiac output was observed, measuring $9.9 \pm 2.7 \mathrm{l} / \mathrm{min}$ as compared to the baseline value, and also as compared to the measurements immediately after opening of the shunt.

In the long term, a gradual increase in left ventricular end-diastolic diameter was observed after two $(45 \pm 4 \mathrm{~mm}$ ), four ( $48 \pm 5 \mathrm{~mm}$ ), and eight weeks (53 $\pm 4 \mathrm{~mm}$ ), reaching a $26 \%$ increase as compared to the baseline measurements $(42 \pm 4 \mathrm{~mm})$.

Other indices of the change in cardiac hemodynamics were obtained by comparing pressure-volume loops at baseline, immediately after the shunt, and after 8 weeks. There was a significant rise in the LVEDV (104 $\pm 27 \mathrm{ml})$ compared to the baseline volume $(75 \pm 26 \mathrm{ml})$. None of the animals showed signs of heart failure or distress, in relation to the AV-shunt. 


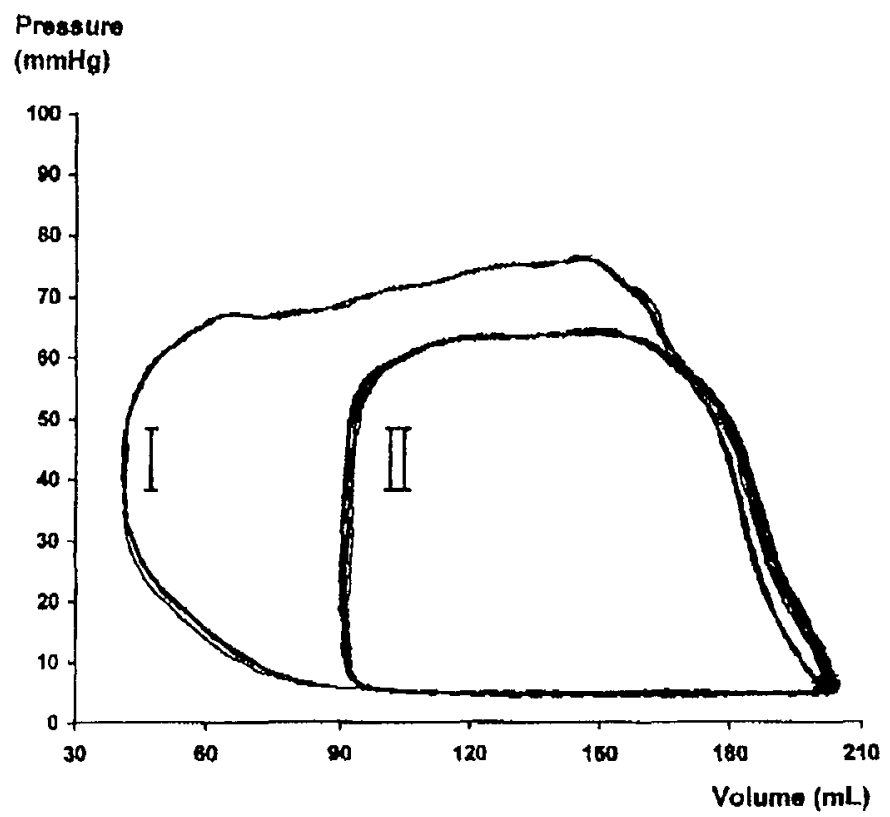

Figure 1. Pressure volume loops obtained during stimulation of the latissimus dorsi muscle every four spontaneous heart beats in a representative animal. The stimulator was programmed at 5 volts with a burst of 6 pulses and a duration of $200 \mathrm{~ms}$, I - $A$ ssisted beats; II Unassisted beats.

\section{Latissimus dorsi muscle stimulation after CMP}

Table 1 presents hemodynamic data obtained at various delays (between the sensing of the QRS and the beginning of the stimulation burst). The delay rendering the worst hemodynamic effect, identified as the lowest stroke volume, was at $25 \mathrm{~ms}$. The individual delays with the best hemodynamic effect were at 75 and $100 \mathrm{~ms}$ in five animals, at $125 \mathrm{~ms}$ in one animal, and at $50 \mathrm{~ms}$ in one animal at a longer burst duration $(220 \mathrm{~ms})$. In most instances, during stimulation at $150 \mathrm{~ms}$ delay, and in one instance of a $125 \mathrm{~ms}$ delay, there was interference with the filling phase of the next beat, and consequently a reduction occurred in the pressure and the ejection fraction (EF). These measurements were excluded from further analyses.

A comparison of unassisted beats versus assisted beats also revealed an increase in systolic blood pressure from $77 \pm 13 \mathrm{mmHg}$ to $87 \pm 12 \mathrm{mmHg}$ (at $10 \mathrm{~V}$ ) and a mean reduction in LVESV of $42 \%$ (from $51 \pm 28 \mathrm{ml}$ to $27 \pm 14 \mathrm{ml}$ ). At the same time, there were no significant changes in left ventricular end- 


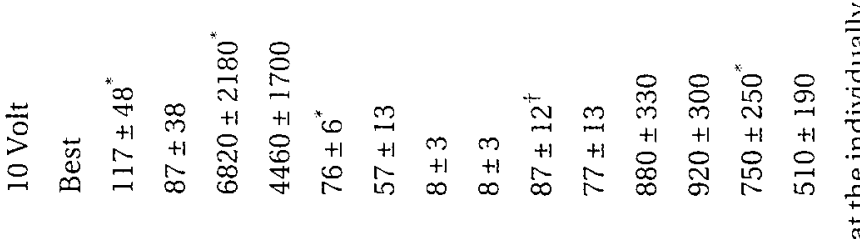

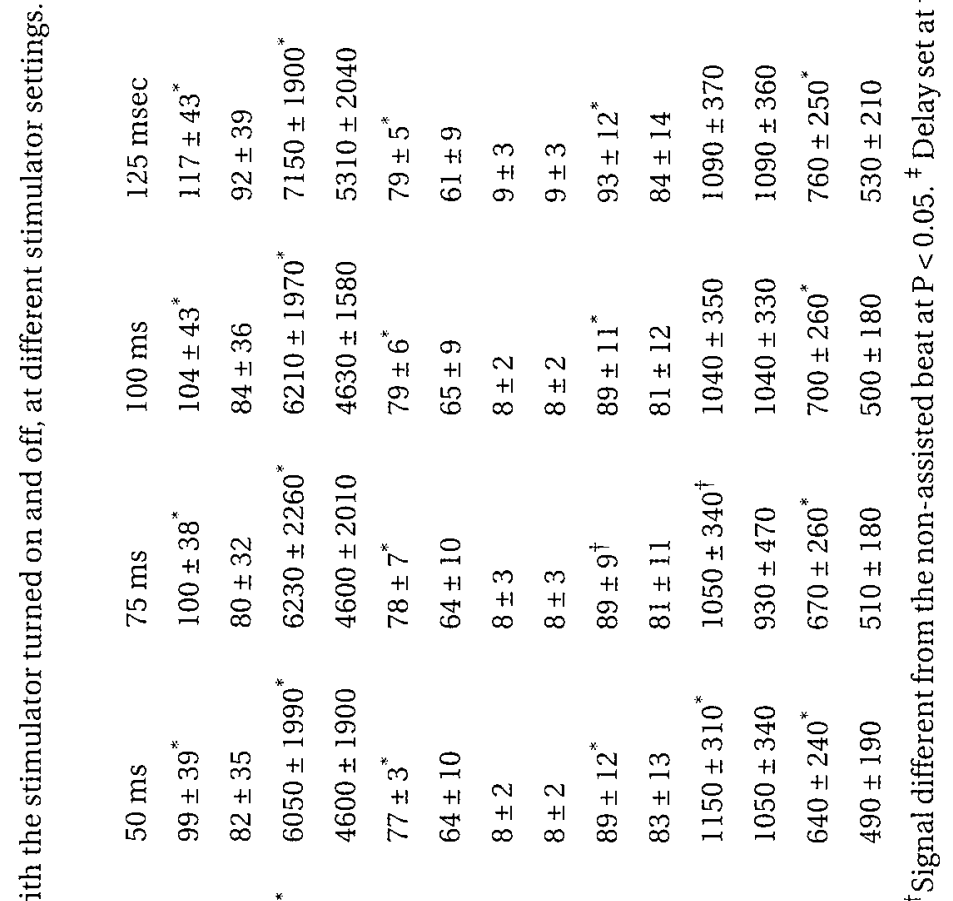

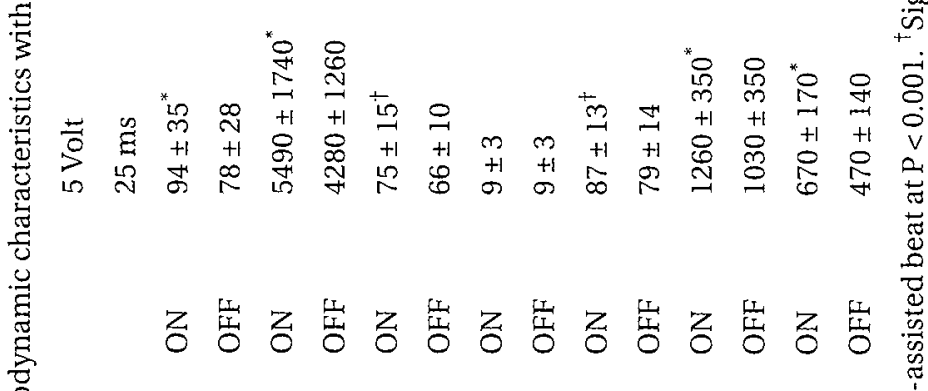

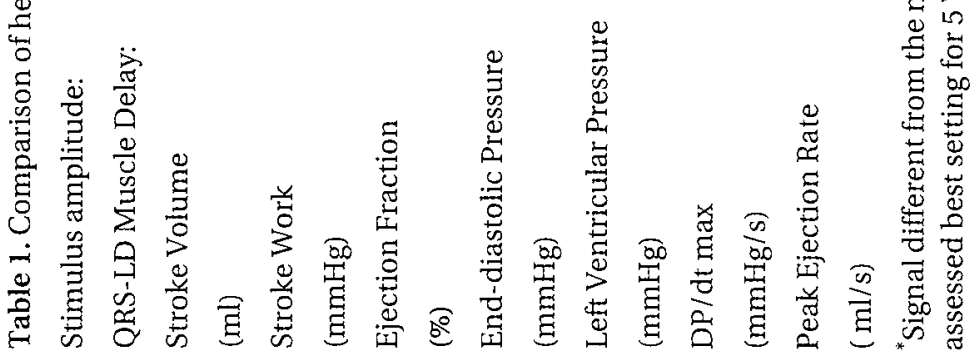


diastolic volume (LVEDV; $151 \pm 55 \mathrm{ml}$ ) or left ventricular end-diastolic pressure (LVEDP $8 \pm 3 \mathrm{mmHg}$ ) in any testing mode.

The pressure volume loop presented in Figure 1 shows a 55\% decrease in LVESV (from $97 \pm 0.7 \mathrm{ml}$ in the unassisted beats to $44 \pm 1.2 \mathrm{ml}$ in the assisted beats). In contrast to these abrupt changes between the assisted beats and the unassisted beats, no change occurred in LVEDP and LVEDV. Stroke volume (SV) appeared to be significantly increased at all settings when assisted beats were compared with unassisted beats (Table 1).

Besides the increase in stroke volume, the stroke work and the ejection fraction were also increased during the assisted beats (Figure 2).

The LVEDP preceding the stimulated heart beat was not directly affected by LD muscle stimulation, whereas systolic LVP was increased by $13 \%$ at 10-Volt testing. The positive $\mathrm{dP} / \mathrm{dt} \max$ was only increased when the LD muscle was stimulated at short delays (25-75 ms). In contrast, the peak ejection rate (PER) was significantly increased at all delay settings during the assisted beats (Figure 2).

Single pulse stimulation every two spontaneous heart beats during 10 minutes of monitoring produced only a small hemodynamic effect accompanied by an increase of LV pressure (5-10 $\mathrm{mmHg}$ ) for a short time (10\% of the duration of systole). However, there was no significant change in the LVEDV and the LVESV at the end of the 10 minute one-pulse stimulation period, nor was there a change 10 minutes later as compared to the baseline.

\section{DISCUSSION}

\section{The Carotid Jugular AV shunt model}

This study demonstrates that the AV shunt heart failure model is capable of producing stable, immediate and long-term high cardiac output (overload) dilatation. None of the eight goats demonstrated clinical signs of pulmonary congestion. Fortunately, these results are in contrast with the high mortality rates $(77 \%)$ and signs of pulmonary congestion occurring commonly within 24 hours after performing the AV shunt in small animals, as reported by Flaim and coworkers (10). This discrepancy is probably due to the relatively small AV shunt, of approximately $10-\mathrm{mm}$, used in our study. Moreover, animals in the present study did not present clinical or hemodynamic signs of heart failure, such as tachycardia or an increase in LVEDP, as was observed with inferior vena cava aortic AV shunt applied in mongrel dogs (11).

Therefore, the described model should be considered a compensatory overload dilatation model rather than a heart failure model. 
$\%$ Improvement S.V.

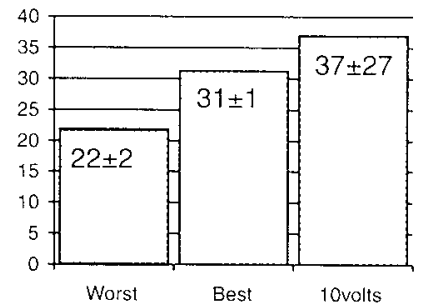

Stimulation setting

$\%$ Improvement S.W.

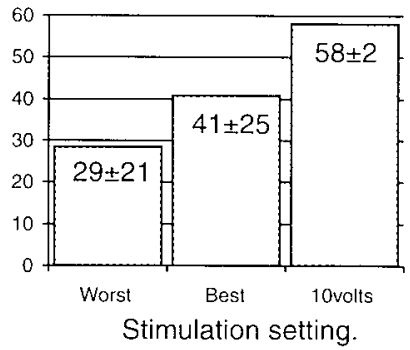

Stimulation setting.
$\%$ Improvement E.F.

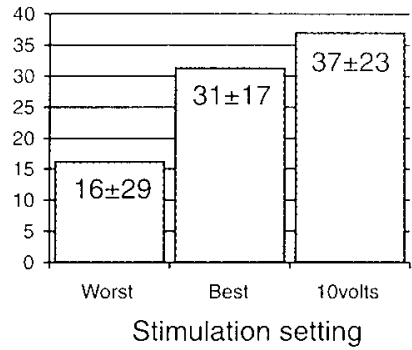

Stimulation setting.

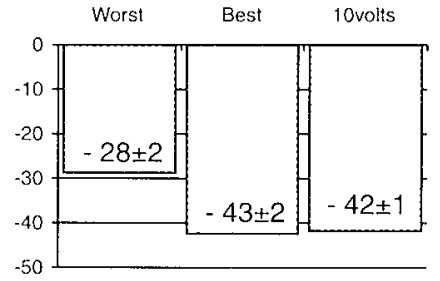

$\%$ Reduction (LVESV).

$\%$ Improvement PER.

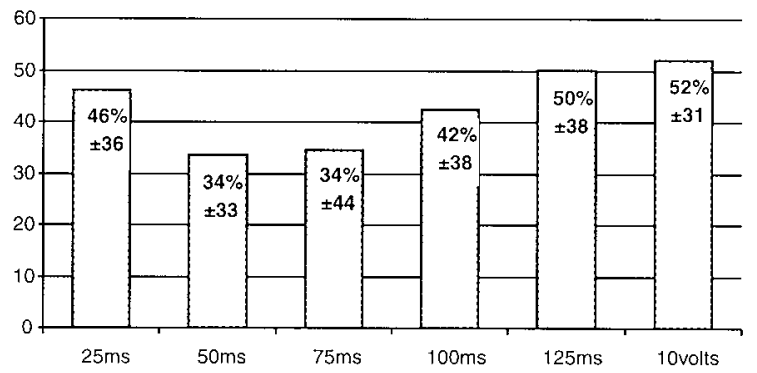

Delay (ms)

Figure 2. Stroke volume (SV), ejection fraction (EF), stroke work (SW), left ventricular endsystolic volume (LVESV), and peak ejection rate (PER) of stimulated beats are compared with non-stimulated beats. The worst and the best settings of the stimulator are shown at the 5 volt as well as the 10 volt setting. The measurements of the PER were performed at different delays between cardiac and LD muscle contraction at a relatively low voltage (25 ms-125 ms, $5 \mathrm{~V}$, and at the hemodynamically best delay at a high voltage (10V). 


\section{LD muscle stimulation}

In the present study, we deliberately chose to begin the LD muscle training protocol immediately after the cardiomyoplasty procedure, hypothesizing that ventricular dilatation and the state of the LD muscle is the key to obtaining systolic augmentation. The clinical data gathered from the work of Schreuder (5) and Kass (6) and from the experimental work of Kaulbach (7) implied that LVEDV reduction (remodeling) starts immediately after the LD muscle wrapping. Reduced LVEDV imply less optimal position on the FrankStarling curve, and therefore it is less suitable for either systolic augmentation in general or LD muscle augmentation in particular. Another explanation is the state of the latissimus dorsi muscle. The reduction in LVEDV after the conditioning protocol as compared to the LVEDV at the wrapping procedure implies a shorter LD muscle and probably a less efficient length for the LD muscle fibers. During the CMP procedure, it is difficult to wrap the LD muscle with significant muscle fiber tension, and the reduction in LV dimensions after the wrapping may cause a yet additional length reduction in the LD skeletal muscle fibers resulting in an inefficient length-tension relation. The nonefficient state of both the LV and the LD muscle at the end of the conditioning period may offer a new explanation for the lack of significant systolic augmentation in most of the clinical experiments.

The state of the freshly raised LD muscle affects the assist function in two ways: the power and speed of contraction decrease considerably after conditioning (12); in fact, the power and speed reduction have been calculated previously (13). Therefore, this factor alone cannot explain the lack of systolic augmentation in both experimental and clinical studies. At the same time, the disconnection of the freshly raised LD muscle from distal collateral circulation leads to acute regional hypoperfusion, which may reduce the LD muscle's power in the acute experiment (14). Despite this regional hypoperfusion, systolic augmentation was considerable in the present study, if compared to the data presented by Yamauchi (15). A possible explanation is that Yamauchi and coworkers applied a LD muscle/heart stimulation contraction ratio of $1: 1$, while we used a 1:4 ratio. Moreover, their use of a non-dilated canine heart may be the reason for obtaining a smaller systolic augmentation.

In our study, stimulator settings were selected to match the clinical protocol, in terms of both the six-pulse burst and the stimulus amplitude (5 V), while the point of the investigation was to examine the effects of the burst delay. Both in the experimental and the clinical settings, rather short delays are reported, of $25 \mathrm{~ms}$ (15) and 45-60 ms (4), respectively. In contrast, in a separate clinical study we reported that the best hemodynamic systolic effect was attained at relatively long delays in patients (16), findings which are in line 
with those of the present study. However, interference with the filling phase of the next beat was observed in most cases testing the $150 \mathrm{~ms}$ delay, and occasionally at a slightly shorter delay of $125 \mathrm{~ms}$. These results emphasize the importance of the exact individual stimulating setting to achieve the optimal effects and to avoid interference with the next heart beat.

In this study, the significant increase in left ventricular $\mathrm{dP} / \mathrm{dt}$ max, which occurred only at the shorter delays of 25-75 ms, did not correlate with an increased SV during LD stimulation. The most likely explanation is that the maximum pressure rise develops just before the opening of the aortic valve and therefore has no effect on cardiac output (16).

\section{The 10 minute one-pulse stimulation}

The reports of Schreuder (5) and Kass (6) demonstrating gradual remodeling of the left ventricle following CMP have given evidence to a new working mechanism of the procedure. The dilated left ventricles in their patients were reduced to about $50 \%$ of the pre-operative volume one year after the operation, as demonstrated by the pressure-volume loops. Although improvement in SV was $20 \%$ at the best setting, the SV improvement was only $3 \%$ at the clinical setting, when comparing assisted heart beats with the preceding unassisted heart beats in a 1: $2 \mathrm{LD}$ muscle/heart contraction ratio. A chronic 1.5\% $\mathrm{SV}$ increase averaged for every heart beat cannot be considered hemodynamically important; but, in ventricular remodeling, this "assist" contributes to the girdling effect of CMP.

The fact that this girdling effect ultimately results in a $50 \%$ reduction in LV volume stresses the difference between active girdling (stimulated muscle) and passive girdling. Vaynblat and coworkers observed that ongoing dilatation could partly be stopped by cardiac binding with a Gore Tex patch (17), as an example of passive girdling.

In an attempt to gather more information about the beginning of the remodeling process, we conducted the 10-minute one-pulse stimulation. This part of the protocol was studied because it resembles the clinical situation at the beginning of the conditioning protocol, two weeks after vascular delay. The conditioning protocol, which requires a period of 8-12 weeks, consists of a gradual increase in the number of pulses per burst, and starts with one pulse per burst for the first two weeks (8). In the present study, there was no detectable significant change in the volume of the left ventricle after 10 minutes of the one pulse stimulation. Therefore, it is most likely that reduction of the LV size occurs as a gradual process that ranges from hours to weeks.

In conclusion, the AV-shunt heart dilatation model is suitable for both demonstrating the ability of the LD muscle to induce pronounced systolic 
augmentation and analyzing the effect of changes in stimulator settings on left ventricular hemodynamic function. It can be anticipated that a dilatation of $26 \%$ (LVEDD), as demonstrated in this study, is essential to achieve pronounced systolic augmentation. Therefore, although the successful LV reduction in patients with dilated cardiomyopathy has a major clinical advantage, it most likely has a negative effect on the ability to demonstrate systolic augmentation.

\section{Acknowledgements}

We thank Mr. Habets and Mr. Van der Nagel for expert biotechnical assistance, Marion Lahaije and Bianca Mourmans for preparing the manuscript, and Telectronics Inc., of Denver, Colorado, for financial support. 


\section{REFERENCES}

1 Cohen-Solal A, Choussat R, Chachques JC et al. Serial assessment of cardiopulmonary exercise capacity after cardiomyoplasty for either ischemic or idiopathic dilated cardiomyoplasty. Am J Cardiol 1996; 77: 623-627.

2 Magovern GJ, Simposon KA. Clinical cardiomyoplasty: Review of the ten-year United States Experience. Ann Thorac Surg 1996; 61:413-419.

3 Delahaye F, Jegaden (), Montagna P, et al. Latissimus dorsi cardiomyoplasty in severe congestive heart failure: The Lyon experience. Journal of Cardiac Surgery, 1991;6: 106-112.

4 Tasdemir O, Vural KM, Kucukaksu SD, et al. Comparative study on cardiomyoplasty patients with the cardiomyostimulator on versus off. Ann Thorac Surg 1996;62: 1708-13.

5 Schreuder JJ, van der Veen FH, van der Velde ET, et al. Left ventricular pressure-volume relationships before and after cardiomyoplasty in patients with heart failure. Circulation 1997;96: 2978-2986.

6 Kass DK, Baughman KL, Pak PH, et al. Reverse remodeling from cardiomyoplasty in human heart failure. Circulation 1995; 91: 2314-2318.

7 Kaulbach HG, Van der Veen FH, Schreuder JJ et al. Dynamic cardiomyoplasty in chronic cardiac overload. Abstract, Circulation 92: I - 379, 1995.

8 Baan J, van der Velde ET, de Bruin HG, et al. Continuous measurement of left ventricular volume in animals and humans by conductance catheter. Circulation 1984; 70 : 812-823.

9 Chachques JC, Grandjean PA, Carpentier A. Latissimus dorsi dynamic cardiomyoplasty. Ann Thorac Surg 1989; 47: 600-604.

10 Flaim SF, Minteer WJ, Nellis SH, Clark DP. Chronic arteriovenous shunt: evaluation of a model for heart failure in rat. Am. J. Physiol 1979;236(5): H698-H704.

11 Porter CB, Walsh RA, Badke FR, O'Rourke RA. Differential effects of diltiazem and nitroprusside on left ventricular function in experimental chronic volume overload. Circulation 1983;68:685-692.

12 Salmons S, Henriksson J. The adaptive response of skeletal muscle to increased use. Muscle and Nerve 1981; 4:94-105.

13 Salmons $\mathrm{S}$ and Jarvis JC. The working capacity of skeletal muscle transformed for use in a cardiac assist role. Mount Kisco NY, Futura Publishing Company, Inc., 1990: 89-104.

14 Isoda S, Yano Y, Jin Y, Walters, Kondo J, Matsumoto A. Influence of a delay on latissimus dorsi muscle flap blood flow. Ann Thorac Surg 1995; 59:632-638.

15 Yamauchi H, Matsui Y, Sutoh Y, Dudra J, Gou M, and Yasuda K. Effects of free latissimus dorsi dynamic cardiomyoplasty on left ventricular function. ASAIO Journal 1997;43: 352-359.

16 Schreuder JJ, van der Veen FH, van der Velde ET, et al. Beat-to-beat analysis of left ventricular pressure-volume relation and stroke volume by conductance catheter and aortic model flow in cardiomyoplasty patients. Circulation 1995;91:2010-2017.

17 Vaynblat M, Chiavarelli M, Shah $\mathrm{HR}$, et al. Cardiac binding in experimental heart failure,. Ann Thorac Surg 1997: 74:81-85. 
The use of the novel non-fluoroscopic three dimensional electroanatomical mapping system to monitor and analyze heart surgery in animal models

Gil Bolotin, Roberto Lorusso, Hans Kaulbach, Jan Schreuder, Gideon Uretzky, Frederik van der Veen 


\section{ABSTRACT}

Background. The new method of three-dimensional electroanatomical mapping was presented as an important tool for cardiac imaging and intervention. We present herein the first use of this technology for the monitoring, analysis, and development of cardiac surgery at the pre-clinical stage.

Methods. The method is based on utilizing a locatable catheter connected to an endocardial mapping and navigating system, to accurately establish the location and orientation of the tip of the mapping catheter and simultaneously record its local electrogram. The three dimensional geometry of the beating cardiac chamber is reconstructed in real time. The system was tested on six goats that underwent dynamic cardiomyoplasty. Three maps of each animal were performed: preoperative, postoperative, and during the stimulation protocol of the skeletal muscle.

Results. The electroanatomical mapping system provided an accurate reconstruction of the three-dimensional beating left ventricle before and after the operation, as well as detailed maps of the left ventricle during the stimulation protocol, which demonstrated a striking geometrical difference between the assisted and the unassisted beats. These geometrical changes are best described by referring to left ventricular long axis movements $\left(22.3^{\circ} \pm 3.8^{\circ} \mathrm{vs}\right.$ $\left.3.4^{\circ} \pm 1.6^{\circ} \mathrm{p}<0.001\right)$, center of mass movements $(10.4 \pm 3.0 \mathrm{~mm}$ vs $3.9 \pm 1.6 \mathrm{~mm}$ $\mathrm{p}<0.005)$ and the changes in upward movement viewed along the base ( $7.9 \pm 1.9 \mathrm{~mm}$ vs $3.6 \pm 1.7 \mathrm{~mm} \mathrm{p}<0.01)$, middle $(13.8 \pm 4.0 \mathrm{~mm}$ vs $7.3 \pm 1.8 \mathrm{~mm}$ $\mathrm{p}<0.005)$, and the apex of the heart $(28.1 \pm 4.5$ vs $5.3 \pm 2.3 \mathrm{~mm} \mathrm{p}<0.001)$.

Conclusions. The three-dimensional electroanatomic mapping system allows detailed reconstruction of the left ventricular geometry and a clear view of the difference between the assisted and the unassisted beats. This novel monitoring system may serve as an important tool for the analysis and development of new techniques in cardiac surgery. 


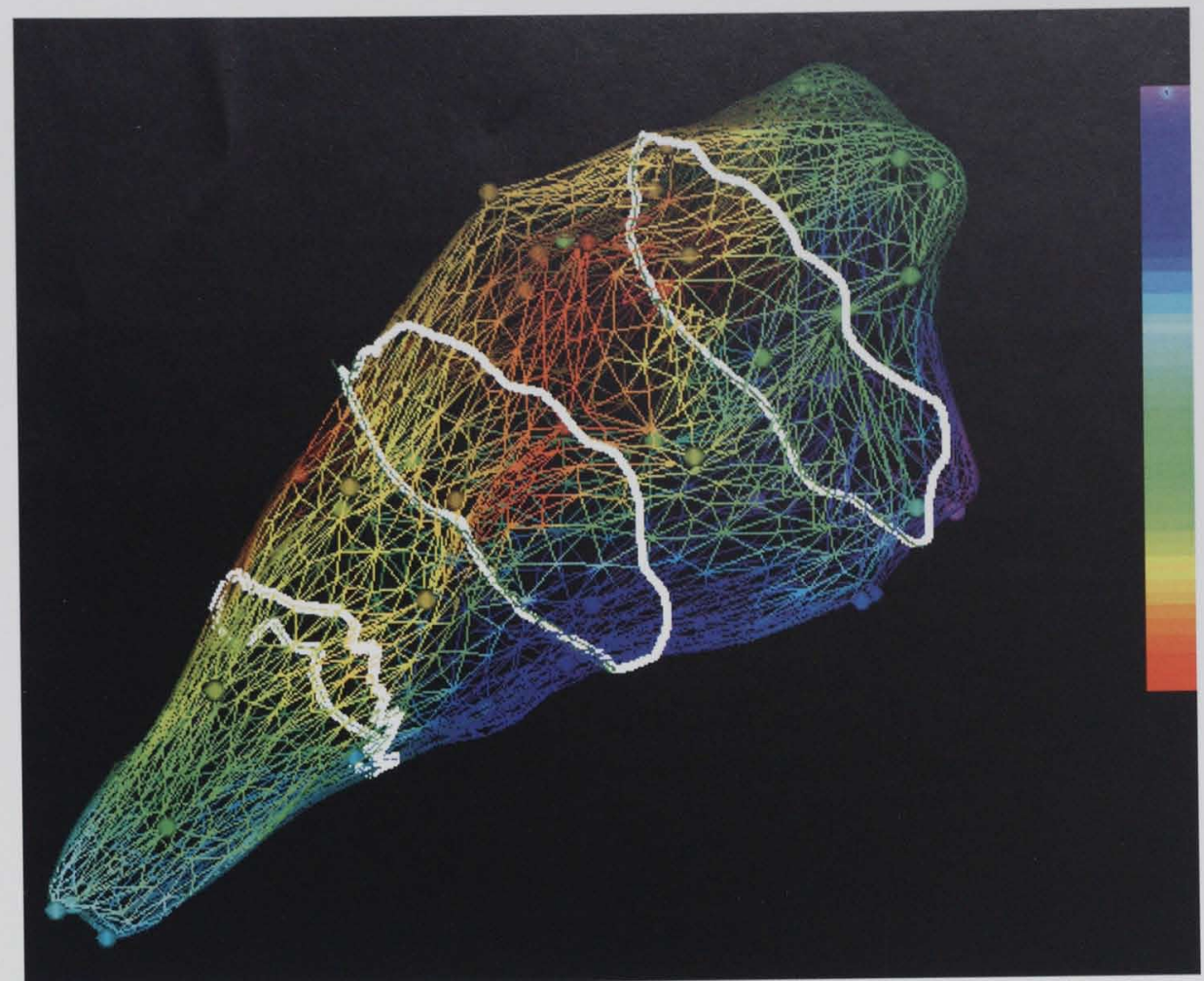

Figure 1. The three cross sections, plotted at 25\%, 50\%, and $75 \%$ of the left ventricle's long axis. The area of these three cross sections was recorded continuously through a normal cardiac cycle as well as during the assisted beats.

\section{INTRODUCTION}

The novel, non-fluoroscopic three-dimensional electroanatomical mapping system has recently been described for the evaluation of left ventricular function $(1,2,3)$. The technique is capable of reconstructing the $3 \mathrm{D}$ beating heart chamber with a superimposed color-coded map of the endocardial electrocardiogram, based on information gathered from sensors at the catheter tip. The electroanatomical mapping system was proven to be highly accurate and reproducible both in vitro and in vivo $(2,3)$. It is currently used clinically for several cardiologic imaging and intervention procedures, including hemodynamic evaluation, distinction between infarcted and healthy myocardial tissue, ablation, and direct myocardial revascularization $(3,4,5,6,7)$. The importance of the imaging and monitoring techniques for the analysis of the pre-clinical cardiac surgery cannot be overestimated. We hypothesize that implementation of this 
new technique at the pre-clinical evaluating phase may reveal new information about the 3D geometrical and hemodynamic effects of cardiac surgery. The cardiomyoplasty procedure has already been subjected to broad pre-clinical and clinical evaluations using a wide range of monitoring and imaging modalities; therefore, we chose to assess the significance of this novel technique by applying it for further pre-clinical evaluation of this procedure.

We present herein the first use of this technology for the analysis of cardiac surgery.

\section{METHODS}

\section{Basic concepts of the electroanatomical mapping technique}

The system is based on the physics principle that a metal coil placed in a magnetic field can generate a current. The size of the current is proportional to the strength of the magnetic field, the location of the coil, and the orientation of the coil in the field (8). The electroanatomical mapping technique was proven to be highly accurate and reproducible both in vitro and in vivo $(9,3)$.

\section{Mapping system components}

The mapping and navigator system (Biosense, I\&I) uses an ultralow electromagnetic field generated from a triangular location pad. A 7 Fr deflectable-tip catheter, containing a miniature location sensor, provides real-time 3D electrical and anatomical maps of the endocardial surface. The system is comprised of five basic components:

1. A triangular location pad with three coils generating an ultralow magnetic field (5 X 10-6 to 5 X 10-5 Tesla units).

2. A stationary reference catheter with a miniature magnetic field sensor, located either in the right heart or externally on the body surface.

3. A 7 Fr navigation mapping catheter, with a deflectable tip and electrodes that provide unipolar or bipolar endocardial signals when inserted into the left ventricle.

4. A miniature passive location sensor within the mapping catheter.

5. The NOGA workstation processing the information obtained from the mapping catheter and constructing the 3D left ventricular geometry.

The electromagnetic field generated by the system contains the information necessary to resolve the location and orientation of the sensor in 6 degrees of freedom. The sensor measures the magnetic field to determine the distance from each of its sources, and to enable the determination of the location and the orientation of the sensor in the space. 
SYSTOLE

APEX

Systole

Before

Area in

$\mathrm{mm}^{2}$

Average 249

SD

$\mathrm{p}$

$$
85
$$$$
0.047
$$

Assisted

sys vs.

sys before

APEX

APEX

Systole

After

Area in

$\mathrm{mm}^{2}$

253

101

0.153

Assisted

sys vs.

sys after

$$
\text { MID }
$$

Systole

MID

Before

Area in $\mathrm{mm}^{2}$

Average $\quad 471$

SD

p

129

0.0004

Assisted

sys vs.

sys before

Assisted

Area in

$\mathrm{mm}^{2}$

412

123

0.0002

Assisted

sys vs.

sys after

$\begin{array}{lll} & \text { BASE } & \text { BASE } \\ & \text { Systole } & \text { Systole } \\ \text { Before } & \text { Assisted } \\ & \text { Area in } & \text { Area in } \\ \mathrm{mm}^{2} & \mathrm{~mm}^{2} \\ \text { Average } & 653 & 653 \\ \mathrm{SD} & 174 & 132 \\ \mathrm{p} & 0.497 & 0.294 \\ & \text { Assisted } & \text { Assisted } \\ & \text { sys vs. } & \text { sys vs. } \\ \text { sys before } & \text { sys after }\end{array}$

145

672

178
Systole

\section{DIASTOLE}

APEX APEX APEX

Diastole Diastole Diastole

Before After-1 After -2

Area in Area in Area in

$\mathrm{mm}^{2} \quad \mathrm{~mm}^{2} \quad \mathrm{~mm}^{2}$

$\begin{array}{lll}389 & 439 & 408\end{array}$

$113 \quad 87 \quad 77$

$0.005 \quad 0.002$

Diastole Diastole

after vs. after -1 vs.

diastole diastole

before after -2

MID MID MID MID

Systole Diastole Diastole Diastole

After Before After-1 After-2

Area in Area in Area in Area in

$\mathrm{mm}^{2} \quad \mathrm{~mm}^{2} \quad \mathrm{~mm}^{2} \quad \mathrm{~mm}^{2}$

$\begin{array}{llll}480 & 687 & 704 & 702\end{array}$

181

184

171

$0.099 \quad 0.444$

Diastole Diastole

after vs. after -1 vs.

diastole diastole

before after -2

BASE BASE BASE BASE

Systole Diastole Diastole Diastole

After Before After -1 After -2

Area in Area in Area in Area in

$\mathrm{mm}^{2} \quad \mathrm{~mm}^{2} \quad \mathrm{~mm}^{2} \quad \mathrm{~mm}^{2}$

991

1026

980

214

232

224

$0.017 \quad 0.006$

Diastole Diastole

after vs. after -3 vs.

diastole diastole

before after -2 


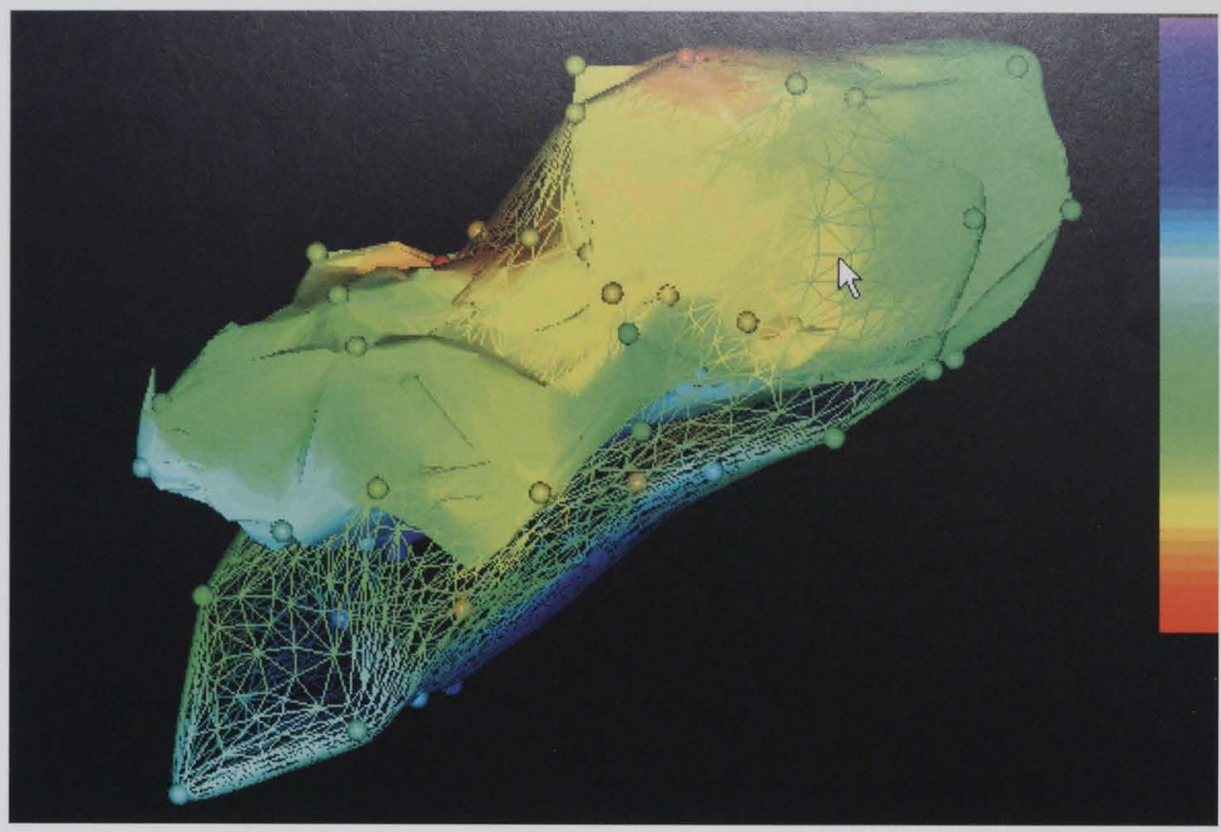

Figure 2. A left anterior oblique 3D reconstruction of the goat's left ventricle, superimposed at two time points: unassisted systole in grid reconstruction and assisted systole in full-color reconstruction.

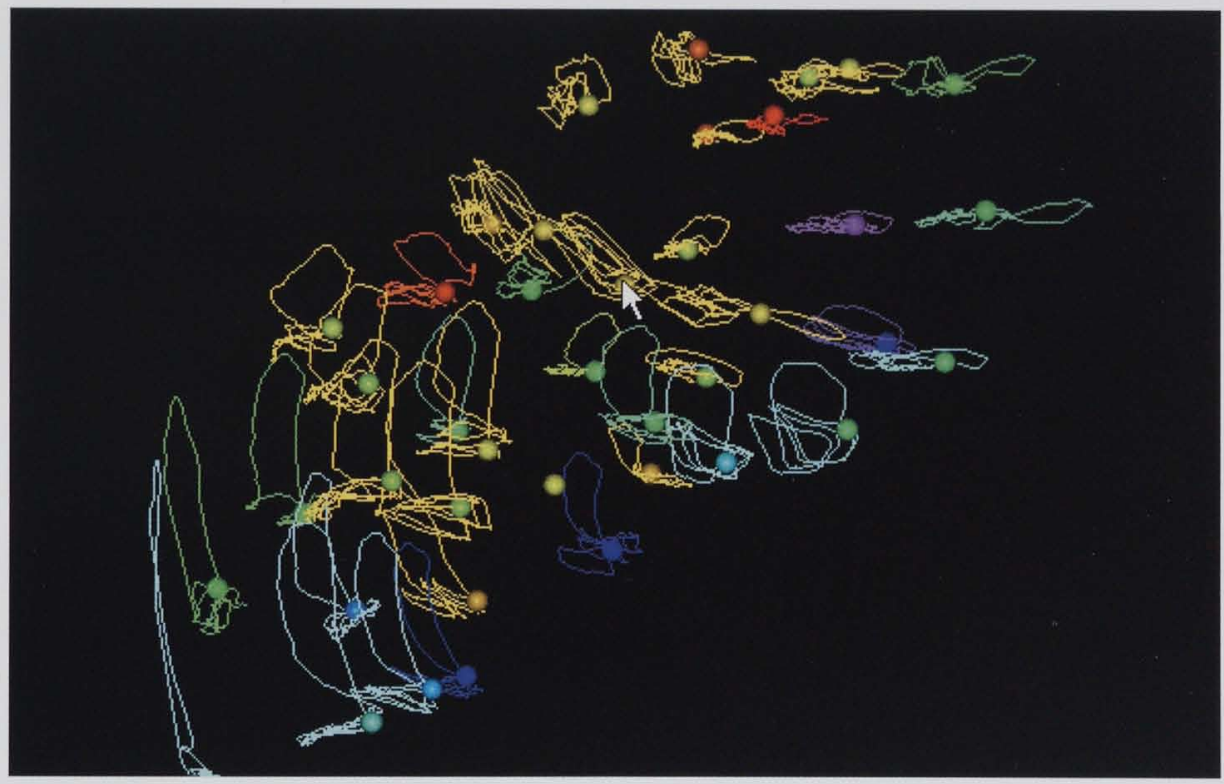

Figure 3. A map of local trajectories showing the motion of each mapped endocardial point during the unassisted and assisted cardiac cycles. 

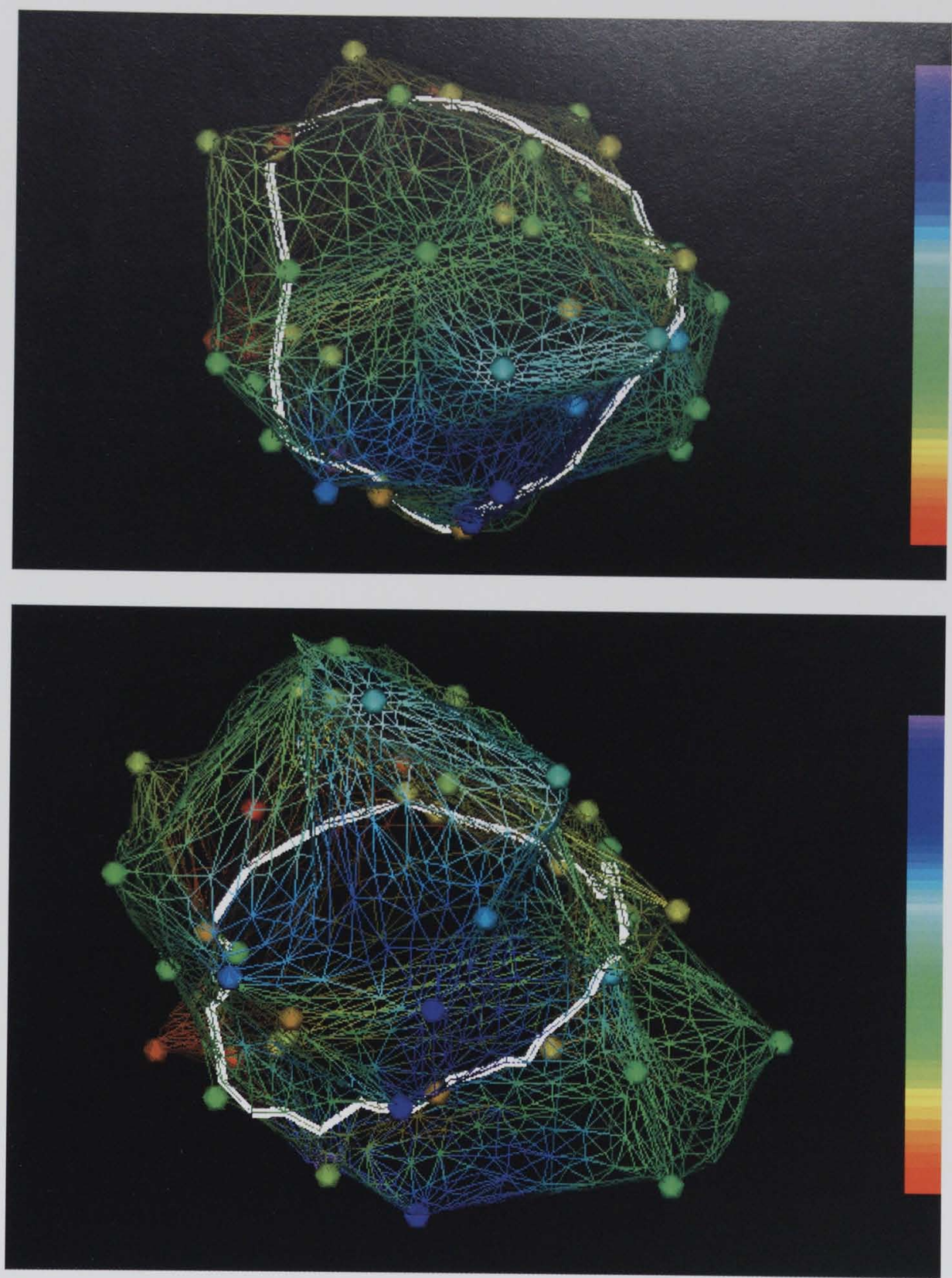

Figure 4 . The cross section area is plotted at the mid part of the heart during unassisted systole (A) and during assisted systole (B). 


\section{Mapping procedure}

The mapping catheter is introduced through a femoral or carotid sheath and placed in the left ventricle. The reference catheter is placed in the right ventricle. The location of the mapping catheter, relative to the reference catheter, is recorded continuously. The tip of the mapping catheter is now moved to multiple left ventricular endocardial sites and the NOGA processor uses a triangular algorithm to reconstruct the left ventricular anatomy. Local activation time and unipolar or bipolar intracardiac electrical recordings are acquired simultaneously. The 3D reconstruction using triangular algorithm that connects the mapped points results in the beating endocardial anatomical map with the local activation time color-coding superimposed on it.

\section{Software developed for the current study}

The need to reconstruct maps which include a beat before and after the assisted beat was solved with the elongation of the sampling time to 3 seconds and the tagging of the system to the stimulator of the skeletal muscle. Since the stimulator was also tagged to the spontaneous heart beats, the result was a synchronous beating 3D reconstruction of at least three heartbeats including the pre and post assisted beats. For the investigation of the different areas of the left ventricle participating in the hemodynamic work, three inner cross sections were plotted on the left ventricular endocardium, at the apex, mid, and base of the heart. The three cross sections were plotted at $25 \%, 50 \%$, and $75 \%$ of the left ventricle long axis respectively (Figure 1). The area of these three cross sections was recorded continuously through a normal cardiac cycle as well as during the assisted beats.

\section{The surgical procedure}

Six goats underwent dynamic cardiomyoplasty. General anesthesia was induced by intravenous sodium thiopental (Penthotal, Thiopentone sodium, Abbot S.p.a.) $15 \mathrm{mg} / \mathrm{kg}$, and maintained after endotracheal intubation with $\mathrm{O}_{2}$ :NO (1:2) and $1.5 \%$ fluothane. Throughout the experiments, lung ventilation was achieved using a positive pressure respirator (Harvard Apparatus Inc., South Natick, MA). Body temperature was kept constant using a heating mattress. Before operation, a single dose of 5,000 U of intravenous heparin was administered.

A left-sided midaxillary incision was performed above the LD muscle and all collateral blood vessels to the distal part of the muscle were coagulated. All attachments of the muscle, except for the axillary pedicle, were disconnected 
to keep the thoracodorsal artery, vein, and nerve intact. Two intramuscular electrodes (Medtronic SP 5590 stimulation leads) were implanted in the upper part of the LD muscle flap, perpendicular to the main branches of the thoracodorsal nerve, as described previously by Chachques and co-workers (11). To assure proper positioning of the electrodes, satisfactory threshold $(0.3$ to $0.6 \mathrm{~V})$ and total recruitment $(1.0$ to $2.5 \mathrm{~V})$ values were obtained after connection of the electrodes to the stimulator system (Medtronic Cardio-Myo Stimulator SP 3076; Medtronic, Kerkrade, The Netherlands). A 5-cm segment of the anterior portion of the second rib, including the periosteum, was then resected to allow transposition of the LD muscle flap into the thorax. The muscle was inserted into the chest cavity, its tendon cut and sutured to the periosteum of the third rib, before closing the thoracic window. The thorax was then opened at the fourth left intercostal space and the pericardium was cut open. A sensing electrode (Medtronic 6500 sensing lead; Medtronic, Kerkrade, The Netherlands) was implanted in the right ventricular wall (adjacent to the septum) and the sensing threshold ( 4.5 to $16.4 \mathrm{mV}$ ) was measured.

The left latissimus dorsi muscle flap was wrapped in a counterclockwise fashion around both ventricles. The muscle was first positioned around the right ventricle and fixed with interrupted sutures near the atrioventricular groove at the base of the heart. Subsequently, the remaining part of the muscle was wrapped around the left ventricle. The distal portion was sutured to the proximal part of the muscle.

\section{Mapping and Statistical Analysis}

Three maps were obtained from each animal preoperatively, postoperatively, and during the stimulation protocol of the skeletal muscle, performed aproximately 45 minutes after accomplishing the wrapping. The pre wrapping (baseline) and post wrapping maps were compared to evaluate the geometrical and hemodynamic effects of the wrapping without stimulation of the skeletal muscle. Later, the cardiomyoplasty-assisted beats were compared to the previous and post unassisted beats, using Student's paired $t$ test. Results are expressed as mean \pm standard deviation. Differences were accepted to be significant at a $p$ value less than 0.05 .

\section{RESULTS}

The three-dimensional reconstructions of the beating left ventricle before and after the operation were accurate, as were the detailed maps obtained during the stimulation protocol. A significant geometrical difference was observed 
between the assisted and the unassisted beats. There were no differences between the superimposed color-coding of the endocardial activation propagation mapped at either the pre- or post wrapping stages, or during stimulation of the skeletal muscle wrapped around the heart.

\section{Comparison of maps obtained before and after the wrapping procedure}

Left ventricular end-diastolic volume reduction was documented in the maps obtained after the wrapping procedure as compared to the pre-operation baseline map $(60.9 \pm 12 \mathrm{ml}$ vs. $80.6 \pm 11 \mathrm{ml}, \mathrm{p}<0.05)$; this was accompanied by a less striking reduction in left ventricular end-systolic volume $(35.8 \pm 7 \mathrm{ml}$ vs. $44.8 \pm 14 \mathrm{ml}, \mathrm{p}=0.09$ ), and non significant changes in ejection fraction $(40 \pm 5 \%$ vs. $45.6 \% \pm 12, \mathrm{p}=0.3$ ).

Changes resulting from the wrapping procedure were demonstrated in three different areas using cross sections of the apex, mid, and base of the heart. We found a significant reduction in the mid part end-diastolic area (779 $\pm 200 \mathrm{~mm}^{2}$ vs. $1011 \pm 80 \mathrm{~mm}^{2}$ before the wrapping, $\mathrm{p}=0.016$ ), and at the base end-diastolic and end-systolic cross section areas $\left(948 \pm 194 \mathrm{~mm}^{2}\right.$ vs. $1245 \pm 33$ $\mathrm{mm}^{2}$ before the wrapping, $\mathrm{p}=0.02 \mathrm{l}$; and $538 \pm 176 \mathrm{~mm}^{2}$ vs. $754 \pm 178 \mathrm{~mm}^{2}$ before the wrapping, $\mathrm{p}=0.0014)$. The cross section of the apex in both systole and diastole and the end-systolic area of the mid part of the heart did not change significantly after the wrapping.

\section{Comparison of assisted beats and unassisted beats}

The striking difference between the assisted and unassisted beats is best understood when studying the three-dimensional left ventricular beating maps reconstructed in all the experiments. A two-dimensional reconstruction of these changes is shown in Figure 2, while the same map was superimposed twice at different points in the cardiac cycle: at normal systole and during the assisted systole thereafter.

The left ventricular long axis was found to move $3.4^{\circ} \pm 1.6^{\circ}$ during normal systole and $22.3^{\circ} \pm 3.8^{\circ}$ during the assisted beats' systole $(\mathrm{p}<0.001)$. The center of mass that was found to move $3.9 \pm 1.6 \mathrm{~mm}$ between normal diastole and systole, moved a mean of $10.4 \pm 3.0 \mathrm{~mm}$ between the assisted systole and the previous diastole $(\mathrm{p}<0.005)$.

To study the regional effects of the assisted left ventricle, different areas in the heart were evaluated by means of mean movement direction of points in the apex, mid, and base of the heart. There was a difference in the number of point movements during assisted as compared to unassisted systole in the three regions, base $(7.9 \pm 1.9$ vs. $3.6 \pm 1.7 \mathrm{~mm}, \mathrm{p}<0.01)$, middle ( $13.8 \pm 4.0$ vs. 7.3 
$\pm 1.8 \mathrm{~mm}, \mathrm{p}<0.005)$, and the apex of the heart $(28.1 \pm 4.5 \mathrm{vs} .5 .3 \pm 2.3 \mathrm{~mm}$, $\mathrm{p}<0.001$ ). The other difference between the regions was in the direction of the point's movements during the assisted beats: the apex moved upward $71^{\circ} \pm$ $7.5^{\circ}$ and theta $-30^{\circ} \pm 13^{\circ}$, the mid part of the heart moved upward $64^{\circ} \pm 16^{\circ}$ and theta $-14^{\circ} \pm 25^{\circ}$, and the base moved upward $11^{\circ} \pm 21^{\circ}$ and theta $-32^{\circ} \pm 26^{\circ}$.

Although the standard deviation was found to be relatively high, the difference in point movement direction was statistically significant between the three areas of the heart $(\mathrm{p}<0.001)$. These relatively complex regional changes can be observed in Figure 3, where the direction of each point movement is indicated.

The ejection fraction of the assisted beats was statistically higher $(39 \pm 7 \%$ vs. $28 \pm 7 \%$ in the unassisted beats, $\mathrm{p}<0.01$ ) and the main change was found to be in the left ventricular end-systolic volumes $(40 \pm 6 \mathrm{ml}$ vs. $46 \pm 8 \mathrm{ml}$ in the unassisted beats, $\mathrm{p}<0.05$ ), while no significant difference was found in the left ventricular end-diastolic volumes $(66 \pm 9 \mathrm{ml}$ vs. $64 \pm 10 \mathrm{ml}$ in the unassisted beats, $p=0.11$ ). To evaluate the relative contribution of the different heart segments during the assisted systole, the area of three inner cross sections around the apex, mid, and base of the heart was studied during normal and assisted systole. It was found that only the mid part of the heart contributes to the higher ejection fraction in the assisted beats (cross section area of $412 \pm 123 \mathrm{~mm}^{2}$ vs. $471 \pm 129 \mathrm{~mm}^{2}$ in the previous unassisted beats, $\mathrm{p}=0.0004$ ). An example of changes in the cross section area at the mid part of the heart is presented in Figure 4. While the cross section area of the base did not change, the cross section area of the apex was paradoxically higher in the assisted beats $\left(276 \pm 104 \mathrm{~mm}^{2}\right.$ vs. $249 \pm 85 \mathrm{~mm}^{2}$ in the previous unassisted beats, $\mathrm{p}=0.047$ ). In contrast, the post assisted diastole cross section area was not significantly changed in the mid part of the heart, while end-diastolic cross section areas were found to be high in the post assisted diastole, both in the apex (cross section area of $439 \pm 87 \mathrm{~mm}^{2}$ vs. $389 \pm 113 \mathrm{~mm}^{2}$ in the pre-assisted

diastole, $\mathrm{p}=0.0053$ ), and base (cross section area of $1026 \pm 232 \mathrm{~mm}^{2}$ vs. $991 \pm$ $214 \mathrm{~mm}^{2}$ in the previous unassisted beats, $\mathrm{p}=0.017$ ).

\section{DISCUSSION}

Several methods are currently in use for the monitoring and analysis of experimental cardiac surgery at the animal pre-clinical stage, including the Swan-Ganz catheter, blood pressure curves, blood flow measurements, echocardiography, and conductance catheters $(10,11,12,13)$. The results in the current study suggest that the electroanatomical mapping technique is capable of demonstrating more data than can be gathered using only one of 
the other imaging modalities, as well as data that is achieved only by the electroanatomical mapping technique.

The clear reduction in the left ventricular end-diastolic volume after the wrapping of the latissimus dorsi muscle was previously described using the conductance catheter technique (14). While the conductance catheter technique is capable of measuring the segmental changes in volume, the electroanatomical mapping technique is capable of demonstrating the regional effects in more dimensions, including changes in cross sectional areas, as in the current study, or by using a calculated fractional shortening of regional endocardial surface (16). The data of the cross section areas in the current study indicate that the reduction in the left-ventricular end-diastolic volume after the wrapping is mainly in the mid and base part of the heart.

The ability of the system to be tagged and to reconstruct the beating left ventricle in $3 \mathrm{D}$ is an important tool for the understanding and analysis of cardiac interventions, in particular if the intervention does not affect every spontaneous beat, as is the case with the IABP, several assist devices, cardiac pacing, and interventions such as aortomyoplasty and cardiomyoplasty. Findings from the present study show that the change in the long axis direction of the left ventricle in the assisted beat during cardiomyoplasty can be clearly observed with this technique. Moreover, this change of the left ventricle long axis may appear as an artifact in other monitoring techniques such as echocardiography and conductance catheter. In chronic situations, adhesions around the skeletal muscle may reduce these long axis movements; however, the current data suggest that this assumption would be best analyzed by the electroanatomical mapping technique. In the current study, movement of points in several areas of the heart was analyzed during the normal systole and the assisted systole. Figure 3 includes all data describing each point movement during a normal systole and the local movements in different parts of the heart as a result of contraction of the skeletal muscle around the left ventricle. Echocardiography is capable of generating three-dimensional geometric information of the heart, and the improvements in 3D as compared to $2 \mathrm{D}$ echocardiography are important in this respect $(16,17)$. However, although several methods were developed to facilitate automatic border detection, the main drawback of echocardiography for experimental use is the subjective bias of the device operator and the interpreter of the echocardiography data $(18,19,20,21)$. Moreover, the accuracy of echocardiography in assessing the left ventricular volumes was found acceptable only when the left ventricle's volumes were at steady state, but insufficient when examining the cardiac chambers at more extreme preload situations $(22,23)$. Obviously the main advantages of the echocardiography technique over the electroanatomical mapping system are that it is non-invasive and allows for much faster 
data acquisition; advantages that are not always significant at the pre clinical experimental stage (24). In the present study, the information from the three cross-section areas of the left ventricle during the normal cardiac cycle and the assisted beats supplies us with new data regarding the squeezing of the left ventricle by the skeletal muscle. The reduction visible in the cross-area of the mid part of the heart during the assisted systole leads us to conclude that this is the part of the heart most affected by cardiomyoplasty. Moreover, since this data can be generated in real-time, the system can serve as a method for improving the wrapping technique. The other significant data gathered from the cross section areas is the increase in the next beat end-diastolic cross section area at the apex and base; data that can be useful when adjustments are made to find the ideal wrapping configuration. It can be hypothesized that the ideal wrapping configuration and stimulation setting should reduce the three cross-section areas during the assisted systole and increase the enddiastolic volumes at the next diastole.

The conductance catheter technology was used for monitoring the realtime effect of cardiomyoplasty in experimental and clinical studies $(13,25)$. The conductance catheter and the electroanatomical mapping techniques are both invasive and were both compared to other modalities and found to be highly accurate (26), However, each technique has its own advantages: while the conductance catheter technique is a real-time beat-to-beat technique, the electroanatomical mapping technique is capable of producing a beat-to-beat analysis of a mean map gathered during a period of 20-30 minutes. This makes the latter technique more vulnerable to animal or patient hemodynamic instability. Moreover, the time needed to accomplish an electroanatomic map is one of the main drawbacks in the use of this technique in the clinical setting, especially in cardiac surgery, since the hemodynamic changes are more frequent and acute and should be evaluated more closely than with a mean map of every 20 minutes. However, with the electroanatomical mapping technique, more data than just pressure volume loops are generated, such as the $3 \mathrm{D}$ reconstruction, the data about each point's movements, and the different heart segments' hemodynamic analysis with the cross section areas.

In the current study no difference was found in the endocardial activation propagation between the pre and post wrapping maps as well as during the activation protocol; however, this data may be very important for surgery that interferes with the endocardial integrity, such as the Batista operation and aneurysmectomy procedure.

In conclusion, the three-dimensional electroanatomical mapping system allows detailed reconstruction of the left ventricular geometry and a clear view of the difference between assisted and unassisted beats. This novel monitoring system should serve as an important tool for the analysis and development of new techniques in cardiac surgery. 


\section{REFERENCES}

l Ben-Haim SA, Osadchy D, Schuster I, Gepstein L, Ilayam G, Josephson ME. Nonfluoroscopic, in vivo navigation and mapping technology. Nat Med 1996;2:13931395.

2 Gepstein L, Hayam G, Ben-Haim SA. A novel method for nonfluoroscopic catheterbased electroanatomical mapping of the heart. In vitro and in vivo accuracy results. Circulation 1997;95:1611-1622.

3 Gepstein L, Hayam G, Shpun S, Ben-Haim SA. Hemodynamic evaluation of the heart with a nonfluoroscopic electroanatomical mapping technique. Circulation 1997;96: 3672-3680.

4 Kornowski R, Hong MK, Gepstein L, Goldstein S, Ellahham S, Ben-IJaim SA, Leon MB. Preliminary animal and clinical experiences using an electroanatomical endocardial mapping procedure to distinguish infarcted from healthy myocardium. Circulation 1998;98:1116-1124.

5 Gepstein L, Evans SJ. Electroanatomical mapping of the heart: Basic concepts and implications for the treatment of cardiac arrhythmias. PACE 1998;21:1268-1278.

6 Nademanee K, Kosar EM. A nonfluoroscopic catheter-based mapping technique to ablate focal ventricular tachycardia. PACE 1998;21:1442-1447.

7 Kornowski R, Hong MK, Leon MB. Current perspectives on direct myocardial revascularization. Am J Cardiol 1998;81(7A):44E-48E.

8 Ben-Haim SA, Osadchy D, Schuster I, Gepstein L, Hayam G, Josephson ME. Nonfluoroscopic, in vivo navigation and mapping technology. Nat Med 1996;2: 1393- 1395.

9 Gepstein L, Hayam G, Ben-Haim SA. $\Lambda$ novel method for nonfluoroscopic catheterbased electroanatomical mapping of the heart. In vitro and in vivo accuracy results. Circulation 1997;95:1611-1622.

10 O'Malley, Smith B, Hamlin R, Nickel J, Nakayama T, MacVicar M, Mann B. A comparison of bolus versus continuous cardiac ouput in an experimental model of heart failure. Crit Care Med 2000;28:1985-1990.

11 Goedje O, Hoeke K, Lichtwarck-Aschoff M, Faltchauser A, Lamm P, Reichart B. Continuous cardiac output by femoral thermodilution calibrated pulse contour analysis: comparison with pulmonary arterial thermodilution. Crit Care Med 1999;27(11):24072412.

12 Cou TM, Sudhir K, Iwanaga S, Chatterjee MB, Yock PG. Measurements of volumetric coronary flow by simultaneous intravascular two-dimensional and Doppler ultrasound: Validation in an animal model. Am Heart J 1994;128:237-243.

13 Lorusso R, Van der Veen FH, Schreuder JJ, Bolotin G, Kaulbach HG, Frietman R, Habets Jo, Van der Nagel T, Wellens HJ. Hemodynamic effects in acute cardiomyoplasty of different wrapped muscle activation times as measured by pressure/volume relation. J Card Surg 1996;11:217-225.

14 Bolotin G, Lorusso R, Schreudrer JJ, Kaulbach H, Uretzky G, Van der Veen FH. Perioperative hemodynamic and geometrical changes of the left ventricle during cardiomyoplasty in goats with dilated left ventricle. Isr J Med Sci 1996;32:911 (abstract).

15 Gepstein L, Goldin A, Lessick J, Hayam G, Shpun S, Schwartz Y, Hakim G, Shofti R, Turgeman A, Kirshenbaum D, Ben-Haim SA. Electromechanical characterization of chronic myocardial infarction in the canine coronary occlusion model. Circulation 1998;98:2055-2064. 
16 Nadkarni SK, Boughner DR, Drangova $M$, Fenster A. Three-dimensional echocardiography: assessment of inter-and intra-operator variability and accuracy in the measurements of left ventricular cavity volume and myocardial mass. Phys Med Biol 2000;45(5):1255-1273.

17 De Castro S, Pandian NG. Three-dimensional echocardiography: clinical relevance and application. Am J Cardiol 1998;81(12A):96G-1026.

18 Sonka M, Liang W, Kjanani P, Allan J, DeJong S, Kerber R, Mckay C. Intracardiac echocardiography: computerized detection of left ventricular borders. Int I Card Imaging 1998;46(6):397-411.

19 Heusch A, Koch JA, Krogmann ON, Korbmacher B, Bourgeois M. Volumetric analysis of the right and left venticle in a porcine heart model: comparison of three-dimensional echocardiography, magnetic resonance imaging and angiocardiography. Lur J Ultrasoud 1999;9(3):245-255.

20 Rodevan O, Bjomerheim R, Ljosland M, Maelıle J, Smith IIJ, Ihlen II. Left atrial volumes assessed by three- and two-dimensional echocardiography compared to MRI estimates. Int J Card Imaging 1999;15(5):397-410.

21 Jiang L, Morrissey R, Handschumacher MD, Vazquez de Prada JA, He J, Picard MII, Weyman $\mathrm{AE}$, Levine $\mathrm{R} \Lambda$. Quantitative three-dimensional reconstruction of left ventricular volume with complete borders detected by acoustic quantification underestimates volume Am Heart J 1996;131:553-559.

22 Chen Ch, Nevo E, Fetics B, Nakayama M, Pak PH, Maughan WL, Kass DA. Comparison of continuous left ventricular volumes by transthoracic two-dimensional digital echo quantification with simultaneous conductance catheter measurements in patients with cardiac disease. Am J Cardiol 1997;80(6):756-761.

23 Gorcsan J III, Denault A, Mandarino WA, Pinsky MR. Left ventricular pressure-volumes relations with transesophageal echocardiographic automated border detection: comparison with conductance-catheter technique. Am Heart J 1996;131(3):544-552.

24 Balestrini L, Fleishman C, Lazoni L, Kisslo J, Resai Bengur A, Sanders SP, Li JS. Real-time 3-dimensional echocardiography evaluation of congenital heart disease. J $\mathrm{Am}$ Soc Echocardiogr 2000;13(3):171-176.

25 Schreuder J], Van der Veen, Van der Velde ET, et al. Beat-to-beat analysis of left ventricular pressure-volume relation and stroke volume by conductance catheter and aortic model flow in cardiomyoplasty patients. Circulation 1995;91:2010-2017.

26 Burkhoff ZD, Van der Valde E, Kass D, Baan J, Maughan W, Sagawa K. Accuracy of volume measurements by conductance catheter in isolated, ejecting canine hearts. Circulation 1985;72(2):440-447. 

Chapter

\section{Acute descending aortomyoplasty induces coronary blood flow augmentation}

Gil Bolotin, Tamir Wolf, Frederik van der Veen, Rona Shofti, Roberto Lorusso, Jan J. Schreuder, Gideon Uretzky

Presented at the European Association for Cardio-Thoracic Surgery, 11th annual meeting, October 1997, Copenhagen, Denmark. 


\begin{abstract}
Background. Aortomyoplasty is a procedure aimed to improve cardiac output in patients suffering from heart failure. Stimulation of the latissimus dorsi muscle around the aorta produces hemodynamic effects similar to those of the intraaortic balloon pump. These may be maintained without the accompanying complications or the need for anticoagulation. The objective of this study was to test the acute effects of aortomyoplasty on coronary artery blood flow.

Methods. Eight mongrel dogs (18 to $30 \mathrm{~kg}$ ) underwent acute descending aortomyoplasty. Several stimulation protocols were applied after wrapping of the latissimus dorsi muscle around the aorta in different surgical configurations. The left anterior descending coronary blood flow was measured using a transonic Doppler flow probe. Left ventricular and aortic pressures, proximal and distal to the aortomyoplasty site, were monitored continuously.

Results. Significant aortic diastolic pressure augmentation was expressed both as an increase in peak values, from $110 \pm 24 \mathrm{~mm} \mathrm{Hg}$ to $120 \pm 24 \mathrm{~mm} \mathrm{Hg}$ (p $<0.001)$ and as an increase in the diastolic integral, from $64 \pm 23 \mathrm{~mm} \mathrm{Hg} \times \mathrm{s}$ to $84 \pm 37 \mathrm{~mm} \mathrm{Hg} \times \mathrm{s}(\mathrm{p}<0.001)$. Concomitantly, peak left anterior descending coronary blood flow increased from $26 \pm 10 \mathrm{~mL} / \mathrm{min}$ to $32 \pm 12 \mathrm{~mL} / \mathrm{min}(\mathrm{p}<$ 0.001 ). This was associated with an increase in the diastolic flow integral from $11 \pm 4 \mathrm{~mL}$ to $14 \pm 6 \mathrm{~mL}(\mathrm{p}<0.001)$.
\end{abstract}

Conclusions. Descending aortomyoplasty induces significant augmentation of coronary blood flow. Optimal timing of muscle stimulation is important in achieving the best assist. This procedure may prove beneficial for end-stage ischemic patients. 
Intraaortic balloon pump (IABP) counterpulsation has proved to be a useful clinical tool in supporting heart failure patients and was found to be especially effective in cases of acute ischemia. The IABP causes a decrease in both peak systolic pressure and myocardial oxygen consumption while simultaneously increasing mean diastolic blood pressure and coronary blood flow (1-3). However, long-term IABP support is not possible due to the occurrence of various complications, such as infection and thromboembolic events (4).

Aortomyoplasty is a surgical approach that aims to mimic the advantageous hemodynamic effects of the IABP and maintain them permanently without the accompanying complications or the need for anticoagulation. Since first described by Chachques and coworkers in 1990 (5), experimental, as well as preliminary clinical data concerning dynamic aortomyoplasty have been gathered $(6,7)$.

Both ascending aortomyoplasty (ie, wrapping of the right latissimus dorsi (LD) muscle around the ascending aorta) and descending aortomyoplasty (ie, wrapping of the left LD muscle around the descending aorta) revealed significant hemodynamic improvements during stimulation of the wrapped LD muscle (7-9). Chachques and associates (5) demonstrated significant diastolic pressure augmentation in goats while the LD muscle was stimulated around the ascending aorta. The same group presented experimental results 12 and 24 months postoperatively using a conditioned LD muscle. Diastolic augmentation was maintained two years after the operation (10), implying a potential long-term benefit for patients with heart failure.

One of the main advantages of LABP counterpulsation is the increase in coronary blood flow in ischemic patients $(1,3)$. The purpose of this study was to assess coronary blood flow during assistance with descending aortomyoplasty. This was performed by examining four different surgical configurations: the LD muscle wrapped clockwise and counterclockwise around the aorta, both tightly and loosely in both configurations.

\section{MATERIALS AND METHODS}

Eight mongrel dogs weighing 13 to $30 \mathrm{~kg}$ were used for this study. The experiments were performed in accordance with the "Guide for the Care and Use of Laboratory Animals" published by the National Institutes of Health (NIH publication 85-23, revised 1985). 


\section{Surgical Procedure}

General anesthesia was induced by intravenous sodium thiopental (Penthotal, Thiopentone sodium, Abbot S.p.a.) $15 \mathrm{mg} / \mathrm{kg}$, and maintained after endotracheal intubation with $\mathrm{O}_{2}: \mathrm{NO}(1: 2)$ and $1.5 \%$ fluothane. Throughout the experiments lung ventilation was achieved using a positive pressure respirator (Harvard Apparatus Inc., South Natick, MA). Body temperature was kept constant using a heating mattress. Before surgery, a single dose of 5,000 U of intravenous heparin was administered.

A left-sided midaxillary incision was performed above the LD muscle and all collateral blood vessels to the distal part of the muscle were coagulated. All attachments of the muscle, except for the axillary pedicle, were disconnected in order to keep the thoracodorsal artery, vein, and nerve intact. Two intramuscular electrodes (Medtronic SP 5590 stimulation leads) were implanted in the upper part of the LD muscle flap, perpendicular to the main branches of the thoracodorsal nerve, as described previously by Chachques and co-workers (11). To assure proper positioning of the electrodes, satisfactory threshold $(0.3$ to $0.6 \mathrm{~V})$ and total recruitment ( 1.0 to $2.5 \mathrm{~V}$ ) values were obtained after connection of the electrodes to the stimulator system (Medtronic Cardio-Myo Stimulator SP 3076; Medtronic, Kerkrade, The Netherlands). A $5-\mathrm{cm}$ segment of the anterior portion of the second rib, including the periosteum, was then resected to allow transposition of the LD muscle flap into the thorax. The muscle was inserted into the chest cavity, its tendon cut and sutured to the periosteum of the third rib, before closing the thoracic window. The thorax was then opened at the fourth left intercostal space and the pericardium cut open. A sensing electrode (Medtronic 6500 sensing lead; Medtronic, Kerkrade, The Netherlands) was implanted in the right ventricular wall (adjacent to the septum) and the sensing threshold ( 4.5 to $16.4 \mathrm{mV}$ ) was measured.

A segment of approximately 8 to $10 \mathrm{~cm}$ of the descending aorta ( 1.5 to 2.5 $\mathrm{cm}$ in diameter) distal to the left subclavian artery, was then mobilized. All intercostal arteries arising from the aorta in this particular portion were ligated and divided. The left LD muscle flap was subsequently wrapped around the exposed descending aorta (single layer) and four surgical wrapping configurations (clockwise, counterclockwise, loose, and tight) were applied (throughout all experiments) using Prolene 2-0 sutures (Ethicon Ltd., Edinburgh, Scotland). Tight wrapping was defined as the maximal tightening of the LD muscle around the aorta that did not induce pressure reduction in the distal aorta. Loose wrapping required $1.5 \mathrm{~cm}$ (one finger space) between the aorta and the LD muscle. 


\section{Instrumentation and Data Collection}

A Millar solid-state pressure catheter (Millar Instruments, Houston, TX) was inserted through the left carotid artery and advanced into the left ventricle. Two additional Millar solid-state pressure catheters were inserted through the right and left femoral arteries and advanced into the aorta, proximally and distally to the wrapping site, respectively.

After left thoracotomy, the pericardium was opened and a transonic Doppler flow probe (Transonic Systems, Ithaca, NY) was placed around the left anterior descending (LAD) coronary artery, proximal to the first diagonal, for measurement of coronary blood flow. The electrocardiogram was continuously monitored throughout all experiments.

\section{Latissimus Dorsi Muscle Stimulation}

Stimulation commenced immediately after application of each surgical configuration. The first part of this protocol comprised a six-pulse burst $(5 \mathrm{~V}$, $165 \mu$ s pulse width, $200 \mathrm{~ms}$ burst duration) using different delays (150, 200, 250, 300, 350, 400 and $450 \mathrm{~ms}$ ) after the QRS complex, to cover the entire diastolic range. The stimulation burst was given every third or fourth spontaneous heart beat. Additional measurements were obtained using a six-pulse burst at $10-V$ pulse amplitude, in conjunction with what was considered to be the best delay (based on the maximal increase in coronary blood flow and proximal aortic pressure augmentation) as seen while using the 5 -V stimulation during the first part of the experiment.

\section{Data and Statistical Analysis}

The hemodynamic data was gathered on an IBM personal computer (IBM, Greenock, UK) using CODAS software (DATAQ, Akron, OH). Additional software was designed to detect peak diastolic pressure and coronary blood flow and to calculate diastolic pressure and coronary blood flow curve integrals (area under the curve). Aortomyoplasty-assisted beats were compared to nonassisted beats using Student's paired $t$ test. Results are expressed as mean \pm standard deviation. Differences were accepted to be significant at $p$ value less than 0.05 . 


\section{RESULTS}

\section{Diastolic Pressure Augmentation}

Contraction of the LD muscle around the descending aorta induced diastolic pressure augmentation in the proximal aorta in all of the applied surgical configurations throughout all the experiments. When the delay was shorter (150 ms after the QRS complex), the LD muscle contracted during ventricular systole and before complete closure of the aortic valve. Thus, pressure changes were induced inside the left ventricle (i.e. a higher left ventricular end-systolic pressure) and a change in peak systolic pressure (proximal aorta) was observed, increasing from $122 \pm 6 \mathrm{~mm} \mathrm{Hg}$ in the unassisted beats to $134 \pm$ $6 \mathrm{~mm} \mathrm{Hg}$ in the assisted beats $(\mathrm{p}<0.05)$. Induction of LD muscle contraction during the latter part of ventricular diastole ( $450 \mathrm{~ms}$ after the QRS complex) resulted in a less profound diastolic pressure augmentation. Moreover, this long delay caused partial obstruction of the aorta during the next systole. This phenomenon induced an increase in the afterload during the next beat, and the systolic pressure in the proximal aorta increased from $128 \pm 8 \mathrm{~mm} \mathrm{Hg}$ in the unassisted beats up to $139 \pm 7 \mathrm{~mm} \mathrm{Hg}$ in the assisted beats $(\mathrm{p}<0.001)$. At the same time, distal aortic pressure decreased. The mean diastolic augmentation of the optimal stimulation delay (for each animal) is shown in Tables 1 and 2. This was expressed as an overall increase in the peak diastolic pressure, from $110 \pm 24 \mathrm{~mm} \mathrm{Hg}$ in the unassisted beats up to $120 \pm 24 \mathrm{~mm} \mathrm{Hg}$ in the assisted beats in the proximal aorta $(\mathrm{p}<0.001)$. Pressure augmentation was significantly maintained throughout the diastole, as evidenced by the proximal aortic diastolic pressure integral (area under the pressure curve), which was $64 \pm 23 \mathrm{~mm} \mathrm{Hg} \mathrm{x} s$ in the unassisted beats and $84 \pm 37 \mathrm{~mm} \mathrm{Hg} \mathrm{x} \mathrm{s}$ in the assisted beats $(\mathrm{p}<0.001)$ (Fig. 1).

\section{Coronary Flow Measurement}

Coronary blood flow (LAD) increased after LD muscle stimulation simultaneously with diastolic augmentation. In summation of all configurations, peak coronary flow increased from $26 \pm 10 \mathrm{~mL} / \mathrm{min}$ in the unassisted beats to $32 \pm 12 \mathrm{~mL} / \mathrm{min}$ in the assisted beats $(\mathrm{p}<0.001)$. This increase was associated with a significant increase in the diastolic flow integral (area under the coronary blood flow curve) from $11 \pm 4 \mathrm{~mL}$ to $14 \pm 6 \mathrm{~mL}$ in the assisted beats ( $\mathrm{p}<$ 0.001) (Fig. 2). 
Table 1. Peak proximal aortic diastolic pressures before and during latissimus dorsi muscle stimulation in different surgical configurations.

\begin{tabular}{|c|c|c|c|c|}
\hline \multirow{2}{*}{$\begin{array}{l}\text { Surgical } \\
\text { Configuration }\end{array}$} & \multicolumn{4}{|c|}{ Delay (ms) } \\
\hline & 250 & 300 & 350 & 400 \\
\hline \multicolumn{5}{|c|}{ Clockwise-loose } \\
\hline Nonassisted & $106 \pm 21$ & $112 \pm 26$ & $107 \pm 32$ & $106 \pm 37$ \\
\hline Assisted & $117 \pm 23$ & $120 \pm 25$ & $113 \pm 31$ & $111 \pm 37$ \\
\hline$p$ value $^{\mathrm{i}}$ & $1 \mathrm{E}-07$ & $1 E-12$ & $3 \mathrm{E}-10$ & $5 \mathrm{E}-05$ \\
\hline \multicolumn{5}{|l|}{ Clockwise-tight } \\
\hline Nonassisted & $101 \pm 10$ & $108 \pm 18$ & $102 \pm 17$ & $110 \pm 21$ \\
\hline Assisted & $119 \pm 21$ & $102 \pm 19$ & $111 \pm 20$ & $116 \pm 23$ \\
\hline$p$ value ${ }^{\mathrm{a}}$ & 0.02 & $2 \mathrm{E}-08$ & $1 \mathrm{E}-07$ & $5 \mathrm{E}-05$ \\
\hline \multicolumn{5}{|c|}{ Counterclockwise } \\
\hline loose & $106 \pm 15$ & $106 \pm 11$ & $105 \pm 11$ & $101 \pm 13$ \\
\hline Nonassisted & $115 \pm 17$ & $113 \pm 12$ & $112 \pm 12$ & $106 \pm 12$ \\
\hline $\begin{array}{l}\text { Assisted } \\
p \text { value }\end{array}$ & $6 \mathrm{E}-07$ & $3 \mathrm{E}-07$ & $3 \mathrm{E}-05$ & $2 \mathrm{E}-02$ \\
\hline \multicolumn{5}{|c|}{ Counterclockwise } \\
\hline tight & $102 \pm 16$ & $106 \pm 11$ & $105 \pm 11$ & $101 \pm 13$ \\
\hline Nonassisted & $115 \pm 17$ & $113 \pm 12$ & $112 \pm 12$ & $106 \pm 12$ \\
\hline $\begin{array}{l}\text { Assisted } \\
p \text { value }^{\mathrm{a}}\end{array}$ & $5 \mathrm{E}-06$ & NS & $1 \mathrm{E}-06$ & $2 \mathrm{E}-08$ \\
\hline
\end{tabular}

All values are given in mm Hg. ${ }^{a} \mathrm{NS}$ indicates not significant $(p>0.05)$.

\section{Surgical Configuration}

The results of the different surgical configurations, namely, clockwise and counterclockwise, loose and tight wrapping of the LD muscle around the descending aorta, are summarized in Tables 1 to 4 (clinically applicable delays; ie, the whole burst was confined to the diastolic phase). Comparing between the tight and loose wrapping techniques reveals a trend toward greater diastolic augmentation of coronary blood flow (Fig. 3) when using the tight configuration, although no statistical significance was observed. Counterclockwise wrapping achieved better coronary blood flow augmentation (Fig. 4), compared to clockwise wrapping of the LD muscle. There was no significant difference in the diastolic pressure augmented between clockwise and counterclockwise wrapping. 
Table 2. Proximal aortic diastolic pressure integral before and during latissimus dorsi muscle stimulation in different surgical configurations.

\begin{tabular}{|c|c|c|c|c|}
\hline \multirow{2}{*}{$\begin{array}{l}\text { Surgical } \\
\text { Configuration }\end{array}$} & \multicolumn{4}{|c|}{ Delay (ms) } \\
\hline & 250 & 300 & 350 & 400 \\
\hline \multicolumn{5}{|l|}{ Clockwise-loose } \\
\hline Nonassisted & $54 \pm 15$ & $63 \pm 20$ & $59 \pm 23$ & $67 \pm 35$ \\
\hline Assisted & $68 \pm 21$ & $82 \pm 30$ & $82 \pm 29$ & $85 \pm 35$ \\
\hline$p$ value $^{\mathrm{a}}$ & $1 \mathrm{E}-10$ & $9 \mathrm{E}-08$ & $3 \mathrm{E}-07$ & $3 E-04$ \\
\hline \multicolumn{5}{|l|}{ Clockwise-tight } \\
\hline Nonassisted & $50 \pm 17$ & $64 \pm 19$ & $60 \pm 13$ & $71 \pm 19$ \\
\hline Assisted & $88 \pm 51$ & $100 \pm 41$ & $77 \pm 20$ & $87 \pm 26$ \\
\hline$p$ value $^{\mathrm{a}}$ & NS & $3 E-04$ & $5 \mathrm{E}-06$ & 0.01 \\
\hline \multicolumn{5}{|c|}{ Counterclockwise } \\
\hline loose & $56 \pm 23$ & $61 \pm 27$ & $48 \pm 32$ & $54 \pm 15$ \\
\hline Nonassisted & $72 \pm 31$ & $71 \pm 37$ & $63 \pm 45$ & $59 \pm 21$ \\
\hline Assisted & $1 \mathrm{E}-05$ & $6 \mathrm{E}-04$ & $5 \mathrm{E}-03$ & NS \\
\hline \multicolumn{5}{|l|}{$p$ value $^{\mathrm{a}}$} \\
\hline \multicolumn{5}{|c|}{ Counterclockwise } \\
\hline tight & $67 \pm 30$ & $66 \pm 32$ & $51 \pm 27$ & $77 \pm 15$ \\
\hline Nonassisted & $90 \pm 50$ & $94 \pm 42$ & $67 \pm 36$ & $96 \pm 28$ \\
\hline Assisted & $5 \mathrm{E}-03$ & $2 \mathrm{E}-05$ & $5 \mathrm{E}-04$ & 0.002 \\
\hline$p$ value ${ }^{\mathrm{a}}$ & & & & \\
\hline
\end{tabular}

All values are given in $\mathrm{mm} \mathrm{Hg} / \mathrm{min}$. ${ }^{a} \mathrm{NS}$ indicates not significant $(p>0.05)$.

\section{Afterload}

Reduction in the end-diastolic pressure in the proximal aorta was demonstrated in most of the experiments, implying a decrease in the left ventricular afterload (Fig. 5). However, no statistical significance was observed and the results were not sufficiently consistent to indicate either a preferred configuration or an effective delay that would lead to afterload reduction. Moreover, in some of the experiments, the LD muscle was not sufficiently relaxed around the aorta during the next systole, implying an untimely aortic narrowing (ie, an increase in the left ventricular afterload). A further tendency toward an increase in the next beat's afterload was observed both when using longer delays and when using the tight wrapping configuration. 

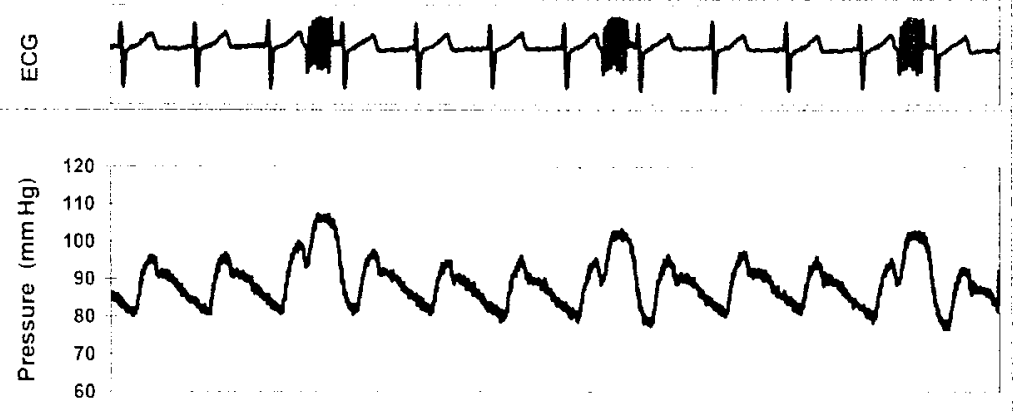

Figure 1. Tracing of proximal aortic pressure illustrating pressure augmentation after latissimus dorsi stimulation (delay, $350 \mathrm{~ms}$ ). (ECG = electrocardiogram.)

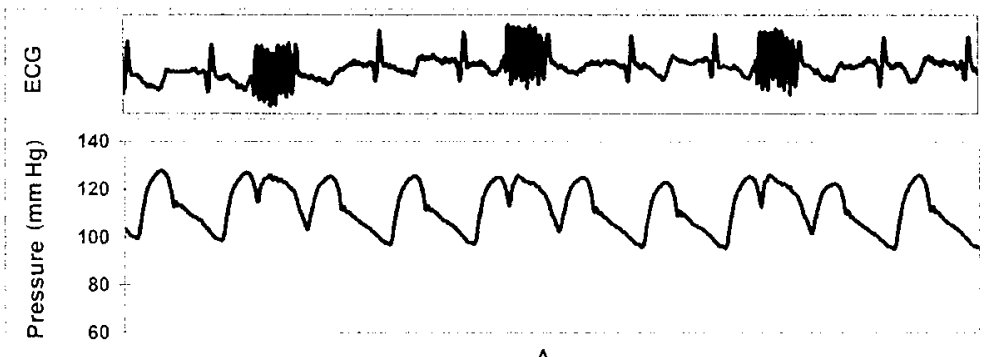

A

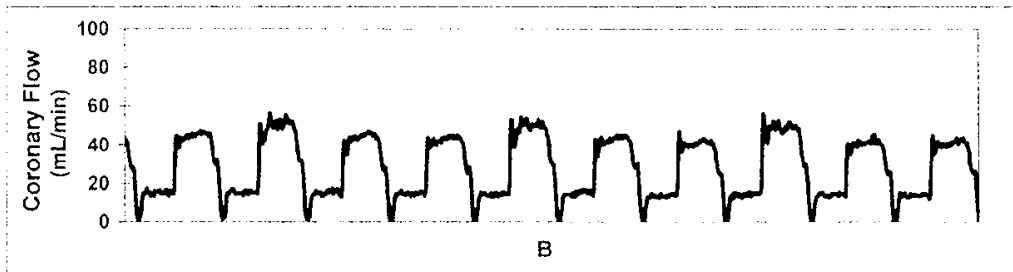

Figure 2. A typical tracing illustrating the concomitant increase in both proximal aortic pressure (A) and coronary blood flow (B) during latissimus dorsi muscle stimulation. (ECG = electrocardiogram.) 


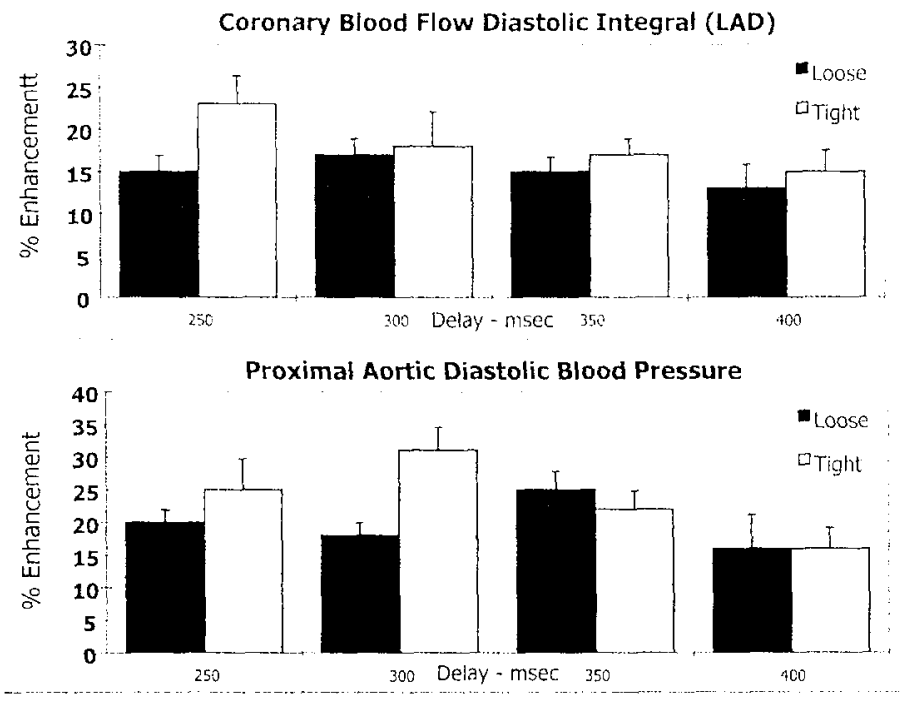

Figure 3. Tight wrapping technique resulted in greater coronary blood flow diastolic integral augmentation. With respect to the proximal aortic pressure area, tight wrapping was more effective throughout the shorter delays. Data are shown as mean \pm standard error of the mean. ( $. A D=$ left anterior descending coronary artery.)

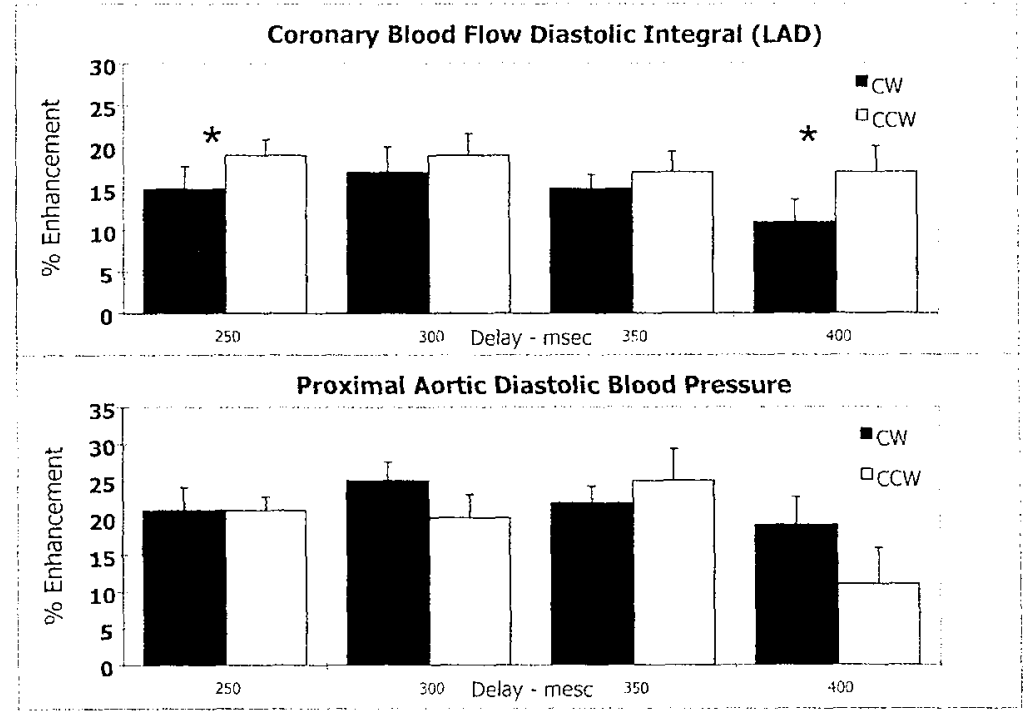

Figure 4. Counterclockwise wrapping of the latissimus dorsi resulted in a more profound coronary blood flow diastolic integral augmentation. Data are shown as mean \pm standard error of the mean $(* p<0.05)$. (CCW $=$ counterclockwise wrapping; $\mathrm{CW}=$ clockwise wrapping; $\mathrm{LAD}=$ left anterior descending coronary artery.) 
Table 3. Peak coronary blood flow before and during latissimus dorsi muscle stimulation in different surgical configurations.

\begin{tabular}{|c|c|c|c|c|}
\hline \multirow{2}{*}{$\begin{array}{l}\text { Surgical } \\
\text { Configuration }\end{array}$} & \multicolumn{4}{|c|}{ Delay (ms) } \\
\hline & 250 & 300 & 350 & 400 \\
\hline \multicolumn{5}{|c|}{ Clockwise-loose } \\
\hline Nonassisted & $30 \pm 10$ & $28 \pm 10$ & $23 \pm 9$ & $27 \pm 10$ \\
\hline Assisted & $34 \pm 11$ & $34 \pm 12$ & $29 \pm 11$ & $30 \pm 11$ \\
\hline$p$ value ${ }^{\mathrm{a}}$ & $5 \mathrm{E}-0.5$ & $3 \mathrm{E}-10$ & $2 \mathrm{E}-07$ & 0.004 \\
\hline \multicolumn{5}{|l|}{ Clockwise-tight } \\
\hline Nonassisted & $21 \pm 2$ & $21 \pm 8$ & $21 \pm 13$ & $25 \pm 11$ \\
\hline Assisted & $26 \pm 1$ & $28 \pm 11$ & $24 \pm 16$ & $30 \pm 15$ \\
\hline$p$ value ${ }^{i l}$ & 0.004 & $4 \mathrm{E}-05$ & 0.006 & 0.05 \\
\hline \multicolumn{5}{|c|}{ Counterclockwise } \\
\hline loose & $31 \pm 9$ & $30 \pm 14$ & $22 \pm 8$ & $19 \pm 8$ \\
\hline Nonassisted & $37 \pm 12$ & $39 \pm 16$ & $30 \pm 12$ & $21 \pm 6$ \\
\hline Assisted & $3 \mathrm{E}-06$ & $2 \mathrm{E}-07$ & $1 \mathrm{E}-04$ & 0.03 \\
\hline \multicolumn{5}{|l|}{$p$ value ${ }^{a}$} \\
\hline \multicolumn{5}{|c|}{ Counterclockwise } \\
\hline tight & $29 \pm 6$ & $23 \pm 7$ & $22 \pm 7$ & $21 \pm 6$ \\
\hline Nonassisted & $38 \pm 7$ & $29 \pm 11$ & $29 \pm 10$ & $31 \pm 10$ \\
\hline Assisted & $3 \mathrm{E}-03$ & $6 \mathrm{E}-05$ & $2 \mathrm{E}-05$ & 2E-05 \\
\hline
\end{tabular}

All values are given in $\mathrm{mL} / \mathrm{min}$. ${ }^{a} \mathrm{NS}$ indicates not significant $(p>0.05)$.

\section{Comment}

Augmentation of coronary blood flow is an important mechanism in IABP support in ischemic patients with heart failure $(1,3)$. A review of the literature reveals a paucity of information regarding coronary blood flow in aortomyoplasty counterpulsation. Cardone and associates (12) induced regional ischemia with LAD coronary artery occlusion in a canine model. They observed an improvement in regional wall motion during counterpulsation with descending aortomyoplasty. These improvements might represent the favorable effects of aortomyoplasty on coronary blood flow. Cernaianu and colleagues (13) compared the use of the LD muscle with that of the serratus anterior muscle in chronic descending aortomyoplasty. They used a model of 
Table 4. Coronary blood flow diastolic integral before and during latissimus ciorsi muscle stimulation in different surgical configurations.

Surgical

Delay (ms)

Configuration

250

300

350

400

Clockwise-loose

Nonassisted

$12 \pm 3$

$11 \pm 4$

$10 \pm 4$

$12 \pm 4$

Assisted

$14 \pm 4$

$14 \pm 5$

$11 \pm 5$

$13 \pm 4$

$p$ value ${ }^{a}$

2E-05

$5 \mathrm{E}-08$

1E-06

0.002

Clockwise-tight

Nonassisted

$13 \pm 1$

$11 \pm 4$

$8 \pm 4$

$11 \pm 4$

Assisted

$16 \pm 1$

$14 \pm 7$

$10 \pm 5$

$12 \pm 6$

$p$ value ${ }^{\mathrm{i}}$

0.022

2E-03

6E-05

$2 \mathrm{E}-03$

Counterclockwise

loose

Nonassisted

$13 \pm 4$

$14 \pm 8$

$9 \pm 3$

$7 \pm 2$

Assisted

$15 \pm 4$

$17 \pm 8$

$11 \pm 5$

$8 \pm 3$

$p$ value ${ }^{a}$

2E-06

3E-05

2e-03

0.006

Counterclockwise

tight

Nonassisted

Assisted

$p$ value ${ }^{\mathrm{a}}$
$13 \pm 7$

$18 \pm 9$

2E-03

$11 \pm 6$

$14 \pm 8$

$6 \mathrm{E}-03$
$9 \pm 4$

$12 \pm 6$

2E-03
$10 \pm 6$

$13 \pm 7$

0.01

All values are given in $\mathrm{mL} / \mathrm{min}$. ${ }^{\mathrm{a}} \mathrm{NS}$ indicates not significant $(p>0.05)$.

ischemia induced by external LAD occlusion in alpine goats. In both normal and ischemic hearts, stroke volume and left ventricular stroke work index increased significantly during LD muscle stimulation. However, no significant increase in mean LAD flow was observed in either the normal or the ischemic heart.

Several methodological differences exist between the studies performed by the groups of Cardone and Cernaianu and our current study. Both compared the hemodynamic profile obtained during an unassisted time period with that of a period assisted by LD muscle stimulation every spontaneous heart beat. Comparison of hemodynamic effects was performed in a beat-to-beat manner between the assisted beats and the corresponding previous unassisted beats. Use of a constant delay of $400 \mathrm{~ms}$ after the R wave and a 1:1 stimu- 


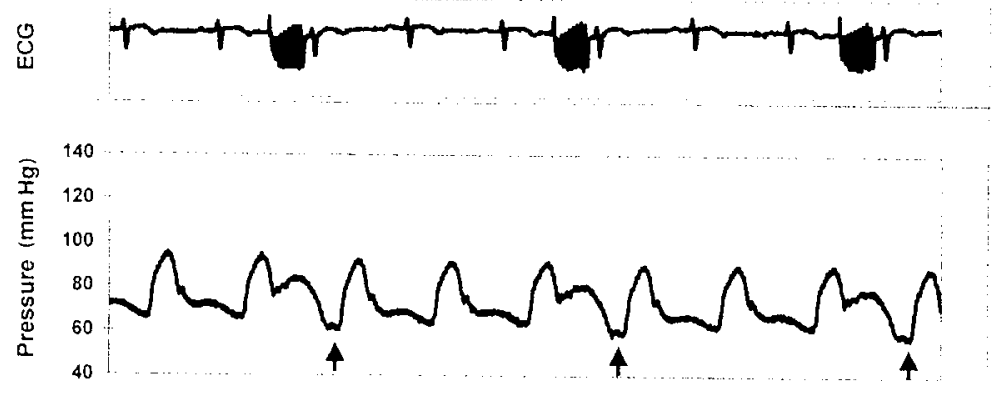

Figure 5. An illustration of proximal aortic pressure afterload reduction (delay, $250 \mathrm{~ms}) .(\mathrm{ECG}=$ electrocardiogram.)

lation protocol may be responsible for the nonsignificant increases observed by Cernaianu and coworkers in the LAD blood flow. Moreover, the stimulation protocol used herein (ie, of either 1:3 or 1:4 stimulation) is in accordance with the clinical setting, and therefore, may accurately resemble the possible hemodynamic advantage. Tailoring these variables for compatibility with the specific heart rate of each animal and conducting a beat-to-beat comparison enables us to ascertain the optimal stimulation parameters for each animal, thus refining the procedure.

The results of the current study demonstrate a significant increase in the LAD blood flow induced by contraction of the LD muscle wrapped around the descending aorta. Simultaneously with this increase in coronary blood flow, diastolic pressure augmentation in the proximal aorta was observed, as described previously in other studies $(14,15)$.

We found that the delay between the QRS and the stimulation of the muscle is crucial to the diastolic augmentation, with respect to the animal's heart rate ( 110 to 140 beats $/ \mathrm{min}$ ). A delay of $450 \mathrm{~ms}$ appeared to be too long, implying the LD muscle was still in a contractile state around the aorta when the next beat's systole was initiated. Thus, the next beat's afterload was higher. Delays of less than $250 \mathrm{~ms}$ caused occlusion of the aorta, indicating a sudden increase in the afterload before the end of the systolic phase. There exists a time window throughout which stimulation of the $\mathrm{LD}$ muscle is hemodynamically optimal. Deviation from this time window, in either direction, produces transient aortic constriction, resulting in a high afterload. However, this optimal delay varies with heart rate, underlying the importance of a physiologic stimulator, which includes the ability to adjust the stimulatory delay and duration in conjunction with the patient's changing heart rate. 
Another important goal regarding the efficacy of aortomyoplasty is to find the optimal surgical configuration. Chacques and colleagues $(5,7,10)$ demonstrated diastolic pressure augmentation in ascending aortomyoplasty. They succeeded in enhancing counterpulsation effects by enlarging the ascending aorta with a pericardial patch. Pattison and coworkers (8) reported significant diastolic augmentation using the LD wrap around the descending aorta. In the current study, effective diastolic pressure and coronary blood flow augmentation were demonstrated in both clockwise and counterclockwise wrapping techniques. However, the increase in coronary blood flow was significantly higher in counterclockwise wrapping as compared to clockwise wrapping. This difference may be attributable to the change in orientation of the LD muscle myofilaments relative to the descending aorta. In addition to the increase in coronary blood flow, afterload reduction is an important factor in the mechanism of counterpulsation. Hymes and associates (15) noted the pivotal role of afterload reduction in aortomyoplasty; however, in their acute canine model, no afterload reduction was demonstrated. Slow LD muscle relaxation may hinder afterload reduction. Thus, we hypothesized that better afterload reduction may be achieved using a loose, rather than tight, configuration, due to earlier dilatation of the compressed aorta. Nonetheless, no significant advantage concerning afterload reduction was observed using the loose configuration. Tight wrapping induced better coronary blood flow augmentation using different delays, and was superior in inducing pressure augmentation throughout the shorter delays (250 and $300 \mathrm{~ms}$ ). Loose wrapping suggests that a slightly longer delay exists between muscle stimulation and aortic compression.

There are several differences between the experimental model, as described in our study, and a clinical situation. No reduction was observed in the skeletal muscle's force and contractile velocity as demonstrated by Salmons and colleagues $(16,17)$ after muscle condition. As in the acute model presented herein, the LD did not undergo a training period. In addition, the use of a healthy rather than a failing heart model is also not compatible with the clinical situation. However, on the basis of previous studies of aortomyoplasty, one may assume that it is more difficult to achieve augmentation of pressure and coronary blood flow in a healthy heart rather than in a failing heart (9).

In conclusion, acute descending aortomyoplasty has been shown to induce both proximal diastolic pressure and coronary blood flow augmentation. Optimal timing of muscle stimulation is important in achieving the best assist. The diversity of results obtained when applying different surgical configurations, as well as the occasional demonstration of afterload reduction, indicates a need for further investigation, and an emphasis on a chronic setting using trained skeletal muscle, to optimize this surgical modality. 


\section{REFERENCES}

1 Muller H, Ayres SM, Conclin EF, et al. The effects of intraaortic counterpulsation on cardiac performance and metabolism in shock with acute myocardial infarcrtion. J Clin Invest 1971;50:1885-1990.

2 Kerber RE, Marcus ML, Ehrhardt J, Abboud FM. Effect of intraaortic balloon counterpulsation on the motion and perfusion of acutely ischemic myocardium. Circulation 1976;53:853-859.

3 Fuchs RM, Brin KP, Brinker JA, Guzman PA, Hvzer RR, Yin FCP. Augmentation of regional coronary blood flow by intraaortic balloon counterpulsation in patients with unstable angina. Circulation 1983;68:117-123.

4 Freed PS, Wasfie T, Zado B, Kantrowitz $A$. Intraaortic balloon pumping for prolonged circulatory support. Am J Cardiol 1988;61:554-557.

5 Chacques JC, Grandjean PA, Fischer EIC, et al. Dynamic aortomyoplasty to assist left ventricular failure. Ann Thorac Surg 1990;49:225-230.

6 Trainini J, Barisani J, Elencwajg B, Cabrera Fischer E, Ahuad A, Roncoroni $\Lambda$. Dynamic aortomyoplasty: clinical experience. J Heart Lung Transplant 1997; 16:882-884.

7 Chachques JC, Radermercker M, Tolan MJ, Fischer EIC, Grandjean PA, Carpentier A. Aortomyoplasty counterpulation: experimental results and early clinical experience. Ann Thorac Surg 1996;61:420-425.

8 Pattison CW, Cumming DVE, Williamson A, et al. Aortic counterpulsation for up to 28 days with autologous latissimus dorsi in sheep. J Thorac Cardiovasc Surg 1991;102: 766-773.

9 Lazzara RR, Trumble DR, Magovern JA. Chronic counterpulsation with descending thoracic aortomyoplasty improved cardiac function in animals with heart failure. J Heart Lung Transplant 1994;13:652-660.

10 Chachques JC, Haab F, Cron $C$, et al. Long-term effects of dynamic aortomyoplasty. Ann Thorac Surg 1994;58:128-134.

11 Chachques JC, Grandjean PA, Carpentier $\Lambda$. Latissimus dorsi dynamic cardiomyoplasty. Ann Thorac surg 1989:47:600-604.

12 Cardone JC, Yoon PD, Trumble DR, Magovern JA. Regional dorsi and serratus anterior descending aortomyoplasty for ischemic cardiac failure. Ann Thorac Surg 1996;61: 426-429.

13 Cernaianu AC, Teimouraz VV, Flum DR, et al. Latissimus dorsi and serratus anterior descending aortomyoplasty for ischemic cardiac failure. Ann Thorac Surg 1995;59: 639-643.

14 Flum DR, Cernaianu AC, Meada R, et al. Descending thoracic aortomyoplasty: a technique for clinical application. Ann Thorac Surg 1996;61:93-98.

15 Hymes W, Hines GL, Lemonick D, Sabini G, Wehbe U. Extraaortic counterpulsation with a latissimus dorsi flap: hemodynamic effects in a heart failure model. J Cardiac Surg 1991;6(supp):184-188.

16 Salmons S, Vrbova G. The influence of activity on some contractile characteristics of mammalian fast and slow muscle. J Physiol Lond 1969;201:535-549.

17 Salmons S, Henriksson J. The adaptive response of skeletal muscle to increased use. Muscle \& Nerve 1981;4:94-105. 


\section{Hemodynamic evaluation of descending aortomyoplasty versus intra-aortic balloon pump performed in normal animals: an acute study}

Gil Bolotin, Tamir Wolf, Robert Sachner, Tamir Wolf, Frederik van der Veen, Rona Shofti, Roberto Lorusso, Jan J. Schreuder, Gideon Uretzky

Presented at the European Association for Cardio-Thoracic Surgery, 12th annual meeting, October, 1999, Glasgow, Scotland.

Published: European Journal of cardio-Thoracic Surgery 2001;19:174-178 


\begin{abstract}
Objective. Aortomyoplasty is a surgical procedure that aims to induce hemodynamic benefits similar to those of the intra-aortic-balloon-pump (IABP). The objective of this study was to compare the coronary blood flow augmentation and afterload reduction produced by IABP and descending aortomyoplasty counterpulsation.

Methods. From a series of fifteen mongrel dogs (18-35 kg), 8 underwent acute descending aortomyoplasty and 7 had IABP application. Left anterior descending (LAD) coronary artery blood flow was measured using a Doppler flow probe. Left ventricular pressure in addition to aortic pressures both proximal and distal to either the aortomyoplasty site or the IABP position were monitored continuously. All experiments were acute and performed in normal hearts.

Results. Descending aortomyoplasty induced a $27 \%$ increase in the LAD blood flow integral during assisted beats $(14.0 \pm 6 \mathrm{ml} / \mathrm{min}$ integral compared to 10.8 $\pm 4 \mathrm{ml} / \mathrm{min}$ integral in unassisted beats $[\mathrm{P}<0.001])$. This was comparable to an $18 \%$ rise in the LAD blood flow integral during IABP counterpulsation (from $8.6 \pm 3 \mathrm{ml} / \mathrm{min}$ to $10.2 \pm 4 \mathrm{ml} / \mathrm{min}[P<0.001])$. Conversely, while IABP counterpulsation reduced the left ventricular afterload by $16 \%$ (from $102 \pm 23 \mathrm{mmHg}$ to $86 \pm 26 \mathrm{mmHg}[\mathrm{P}<0.001])$, descending aortomyoplasty did not result in afterload reduction.

Conclusions. Descending aortomyoplasty produces coronary blood flow augmentation comparable to that achieved by the IABP. This may be important for end-stage ischemic patients. However, afterload reduction achieved by the IABP was not reproduced during descending aortomyoplasty counterpulsation. The surgical technique of descending aortomyoplasty should be modified to attain afterload reduction, thus improving treatment for congestive heart failure patients.
\end{abstract}

Key words. Heart Failure, Skeletal Muscle, Aortomyoplasty, IABP 
IABP is an important tool for the treatment of stage IV ischemic and/or heart failure patients (1). The two main hemodynamic benefits of the IABP are coronary blood flow augmentation and afterload reduction (the difference between end-diastolic pressure during an assisted beat compared to that during an unassisted beat and the reduction in the next beat's systolic pressure) (2). However, long-term IABP support is not feasible due to the occurrence of infection and thromboembolic events (3). Aortomyoplasty is a surgical technique that aims to achieve and maintain the hemodynamic benefits of IABP as a chronic treatment, without the morbidity associated with IABP counterpulsation (4).

Both ascending aortomyoplasty (i.e., wrapping of the right latissimus dorsi [LD] muscle around the ascending aorta) and descending aortomyoplasty (i.e., wrapping of the left LD muscle around the descending aorta) revealed significant hemodynamic improvements during stimulation of the wrapped LD muscle $(5,6,7)$. Chachques and associates demonstrated significant diastolic pressure augmentation in goats while the $\mathrm{LD}$ muscle was stimulated around the ascending aorta (5). The same group presented experimental results 12 and 24 months postoperatively, using a conditioned LD muscle. Diastolic augmentation was maintained two years after the operation (8), implying potential long-term benefit for heart failure patients.

The purpose of this study was to assess coronary blood flow and afterload reduction during assistance with acute descending aortomyoplasty, as compared to the current gold standard, IABP counterpulsation.

\section{MATERIALS AND METHODS}

Fifteen mongrel dogs weighing 18-35 kg were used for this study. Eight underwent acute descending aortomyoplasty and seven had IABP application. The experiments were performed in accordance with the "Guide for the Care and Use of Laboratory Animals" (9). All the experiments were acute and were performed in normal hearts.

\subsection{Aortomyoplasty Surgical Procedure and IABP Insertion}

Both groups underwent general anesthesia, induced by i.v. sodium thiopental (Penthotal) $15 \mathrm{mg} / \mathrm{kg}$, and maintained following endotrachial intubation with $\mathrm{O}_{2}$ :NO (1:2) and 1.5\% Fluothane. Throughout the experiments, lung ventilation was achieved using a positive pressure respirator (Harvard). Body 
temperature was kept constant using a heating mattress. Prior to surgery, a single dose of $5000 \mathrm{U}$ i.v. heparin was administered.

Aortomyoplasty: A left-sided midaxillary incision was performed above the LD muscle and all collateral blood vessels to the distal part of the muscle were coagulated. All attachments of the muscle were disconnected, except for the axillary pedicle, to keep the thoracodorsal artery, vein, and nerve intact. Two intramuscular electrodes (Medtronic SP 5590 stimulation leads) were implanted in the upper part of the LD muscle flap, perpendicular to the main branches of the thoracodorsal nerve, as described previously by Chachques and coworkers (10). To ensure proper positioning of the electrodes, satisfactory threshold (0.3-0.6 V) and total recruitment $(1.0-2.5 \mathrm{~V})$ values were obtained following connection of the electrodes to the stimulator system (Medtronic Cardio-Myo Stimulator SP 3076). A $5 \mathrm{~cm}$ segment of the anterior portion of the second rib was then resected, including the periosteum, to allow transposition of the LD muscle flap into the thorax. The muscle was inserted into the chest cavity, its tendon cut and sutured to the periosteum of the third rib, prior to closing the thoracic window. The thorax was then opened at the fourth left intercostal space and the pericardium was cut open. A sensing electrode (Medtronic 6500 sensing lead) was implanted in the right ventricular wall (adjacent to the septum) and the sensing threshold (4.5-16.4 $\mathrm{mV}$ ) was measured.

A segment of approximately $8-10 \mathrm{~cm}$ of the descending aorta $(1.5-2.5 \mathrm{~cm}$ in diameter), distal to the left subclavian artery, was then mobilized. All intercostal arteries arising from the aorta in this particular portion were ligated and divided. The left LD muscle flap was subsequently wrapped around the exposed descending aorta (single layer) using Prolene 2/0 sutures. The configuration required tight wrapping of the latissimus dorsi around the aorta in a counter clockwise orientation (best configuration from previous study (11).

The IABP catheter (pediatric Datascope IABP catheter $30 \mathrm{ml}$ ) was inserted through the femoral artery and its exact position was determined using fluoroscopic guidance. The IABP was activated in a 1:3 ratio, to allow comparison between the assisted beats and the previous, unassisted beats. The balloon was inflated at different delays following the QRS complex (from 200 to $400 \mathrm{msec}$ ) and with different inflation duration (from 150 to $250 \mathrm{msec}$ ). The best delay and duration were explored by increments of $50 \mathrm{msec}$ and the decision was made according to the plotted proximal blood pressure curve.

\subsection{Instrumentation and Data Collection}

A Millar (Millar Instruments, Texas, USA) solid state pressure catheter was inserted via the left carotid artery and advanced into the left ventricle (LV). 
The pressure curve of the LV was used to avoid inflation of the IABP or contraction of the skeletal muscle prior to closure of the aortic valve. Two additional solid state pressure catheters were inserted via the right and left femoral arteries and advanced into the aorta, proximally and distally to the wrapping site, respectively. Pressure data presented in this study were measured using the proximal intra-aortic pressure catheter.

Following a left thoracotomy, the pericardium was opened and a Doppler flow probe (Transonic Systems, NY) was placed around the left anterior descending (LAD) coronary artery, proximal to the first diagonal, for measurement of coronary blood flow. Surface ECG was monitored continuously throughout the experiments.

\subsection{LD Muscle Stimulation}

Stimulation commenced following the wrapping of the latissimus dorsi muscle around the aorta. The first part of this protocol comprised a six-pulse burst ( 5 volt, $165 \mu \mathrm{sec}$ pulse width, 150, 200, and $250 \mathrm{msec}$ burst duration) using different delays (150, 200, 250, 300, 350, 400 or $450 \mathrm{msec}$ ) after the QRS complex, in order to cover the entire diastolic range. Best delay and duration were chosen based on the plotted proximal blood pressure curve.

The stimulation burst was applied every third or fourth spontaneous heartbeat.

\subsection{Data and Statistical Analysis}

Hemodynamic data was gathered on an IBM personal computer using CODAS (DATAQ, Akron, OH, USA) and HDAS (Maastricht, The Netherlands) software. Additional software was designed to detect diastolic pressure and coronary blood flow peaks and to calculate their curve integrals (area under the curve). Assisted beats were compared to prior, unassisted beats, using Student's paired $t$ test. Results are expressed as mean $\pm \mathrm{SD}$. The correlation between the three variables: diastolic pressure augmentation integral (i.e., area under the diastolic pressure curve), peak diastolic pressure augmentation (assisted beat compared to unassisted beat), and coronary blood flow augmentation (area under the coronary blood flow curve, assisted beat compared to unassisted beat) was analyzed using Pearson's correlation. Differences were considered significant at a $\mathrm{P}$ value $<0.05$. 
The mean descending aortic diameter was $19 \pm 3 \mathrm{~mm}$ and the mean length of the aortic segment to be wrapped was $92 \pm 6 \mathrm{~mm}$, resulting in $26 \pm 2 \mathrm{ml}$ volume of wrapped descending aorta. No deleterious effect on the aorta was observed during the experiments.

\subsection{Proximal aortic pressure and coronary blood flow measurement}

Descending aortomyoplasty counterpulsation induced a $27 \%$ increase in the LAD coronary blood flow integral $(14 \pm 6 \mathrm{ml} / \mathrm{min}$ in assisted beats compared to $10.8 \pm 4 \mathrm{ml} / \mathrm{min}$ in unassisted beats $[\mathrm{P}<0.001])$. This is higher $(\mathrm{P}=0.022)$ than the $18 \%$ rise in the LAD coronary blood flow integral during the IABP counterpulsation assisted beats $(10.2 \pm 4 \mathrm{ml} / \mathrm{min}$ compared to $8.6 \pm 3 \mathrm{ml} / \mathrm{min}$ in unassisted beats $[\mathrm{P}<0.001])$. In addition to the advantage of aortomyoplasty in terms of coronary blood flow augmentation, the procedure also induced a higher diastolic pressure integral than that attained with the IABP (Table 1).

\subsection{Afterload reduction}

Reduction of end diastolic pressure in the proximal aorta was demonstrated with the IABP in the proper timing settings (Figure 1A), and in some of the aortomyoplasty experiments. However, while the afterload reduction induced by the IABP demonstrated statistical significance (from $102 \pm 23 \mathrm{mmHg}$ to $86 \pm$ $26 \mathrm{mmHg}[\mathrm{P}<0.001])$, descending aortomyoplasty failed to induce repetitive afterload reduction (from $91 \pm 12 \mathrm{mmHg}$ to $93 \pm 14 \mathrm{mmHg}$ [NS], P value between the two groups $<0.001)$. Moreover, in some of the experiments, the LD muscle was not sufficiently relaxed around the aorta during the next systole, implying an aortic narrowing, and a subsequent increase in LV afterload (Figure 1B and 1C).

Another parameter of afterload reduction, namely, the reduction of the following beat's systolic pressure, was not demonstrated in either of the counterpulsatory techniques applied.

\subsection{Correlation between hemodynamic variables}

In the IABP group, a significant correlation was observed between the diastolic pressure augmentation integral and coronary blood flow augmentation (Figure 2). This data was gathered from different stimulation settings in the IABP group, resulting in three or four measurements for each animal. 

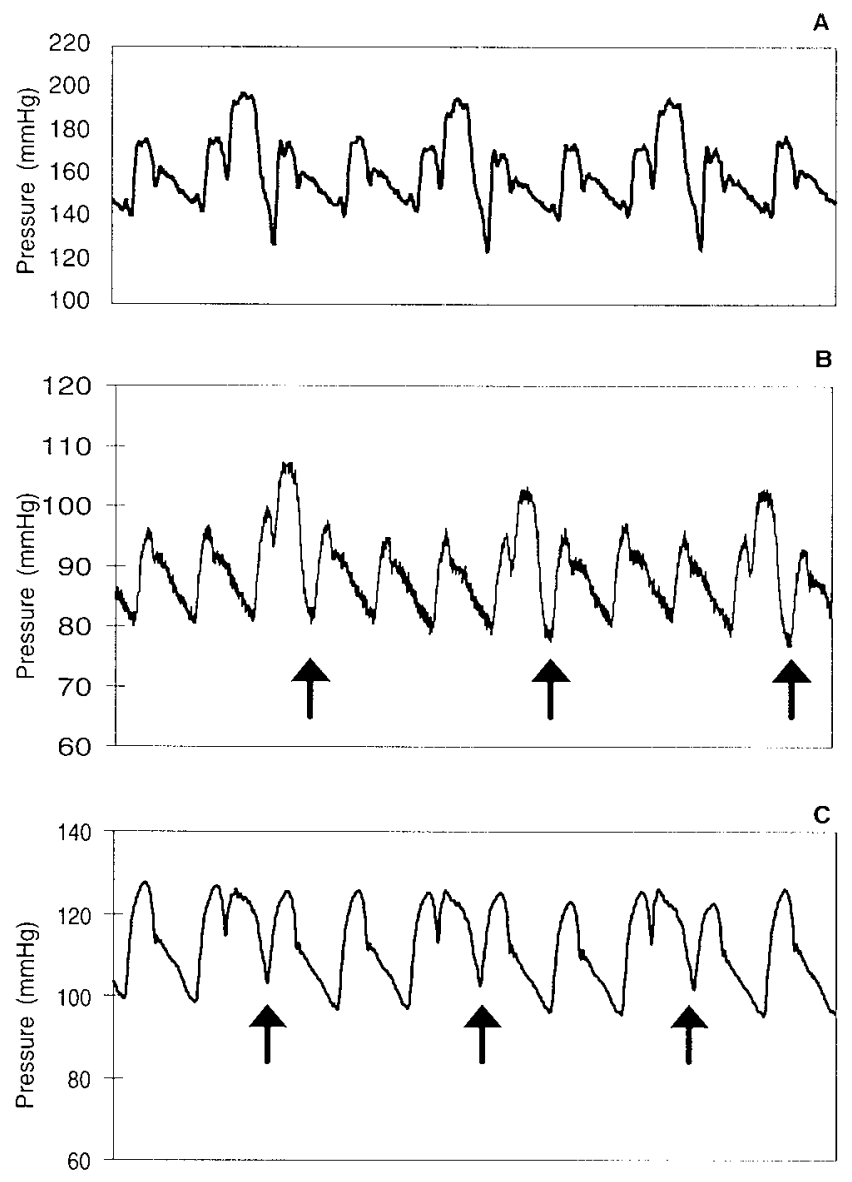

Figure 1. Proximal aortic pressure curves during IABP and descending aortomyoplaşty counterpulsation. Note that while IABP utilization repeatedly caused afterload reduction (A), aortomyoplasty did not induce afterload reduction (B), and in some cases even induced an undesired increase in afterload (C).

However, coronary blood flow was not found to correlate with peak diastolic pressure augmentation. Furthermore, in the descending aortomyoplasty group, no correlation was demonstrated between the hemodynamic variables assessed. 


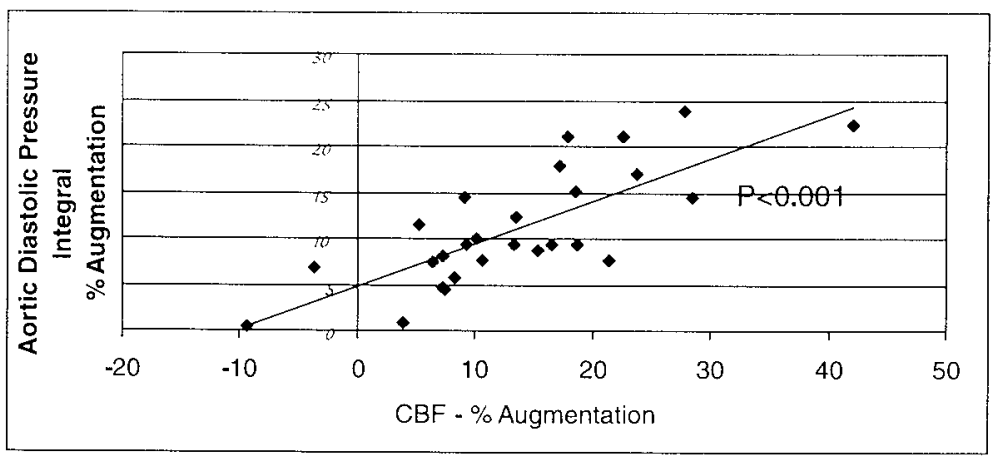

Figure 2. Correlation between augmentation in coronary blood flow integral and proximal aortic diastolic pressure integral.

\section{DISCUSSION}

In the study presented herein, we compared the hemodynamic effects of acute descending aortomyoplasty with the current gold standard, IABP counterpulsation. The results indicate the advantage of the descending aortomyoplasty procedure in achieving coronary blood flow augmentation. In contrast, descending aortomyoplasty did not induce aortic end diastolic pressure reduction, a major component of afterload reduction.

\subsection{Proximal aortic pressure and coronary blood flow measurement}

The advantage of coronary blood flow augmentation in stenotic vessels is still under debate in the literature $(12,13)$. Several authors demonstrate its importance, especially for compromised left ventricular contraction, and in combination with drug-induced reduction of coronary resistance $(14,15)$. In the present study, the coronary blood flow augmentation induced by descending aortomyoplasty is encouraging. In our acute animal model, applying the proper surgical configuration resulted in higher coronary blood flow augmentation than that demonstrated in the IABP control group. This finding is most probably due to the higher diastolic pressure integral (area under the curve) achieved with descending aortomyoplasty. Both findings (coronary blood flow and diastolic pressure integral augmentation) result from the direction of the muscle fibers, and therefore the contraction, which squeezes blood retrogradely into the coronary arteries. This may indicate that the very mechanism of pressure augmentation in the aorta, and not solely the extent (peak) of its enhancement, is responsible for the augmentation of coronary blood flow. 
The higher coronary blood flow augmentation camnot be explained by the volume of the aorta wrapped by the muscle, since this volume was approximately $26 \mathrm{ml}$, smaller then that of the IABP catheter (mean aortic diameter is $1.9 \mathrm{~cm}$ and the wrapped segment can be up to $9.2 \mathrm{~cm}$ in length).

\subsection{Afterload reduction}

The lack of afterload reduction in the aortomyoplasty group can be explained by the relaxation rate of the latissimus dorsi muscle, which may be too slow to induce a measurable decrease in the aortic pressure, such as that produced by the active deflation of the IABP. Proximal aortic end-diastolic pressure reduction was clearly obtained in some of the aortomyoplasty experiments; however, in the majority of the experiments, end-diastolic pressure reduction was not induced, despite careful adjusting of the stimulation settings. This inconsistency may be resolved by modifying the surgical configuration. Two surgical strategies that merit consideration are the ascending aortomyoplasty technique and the introduction of a recoil device between the aorta and the skeletal muscle.

The diameter of the descending aorta in both groups was relatively small (mean $1.9 \mathrm{~cm}$ ). This constitutes a hemodynamic advantage to the IABP group (higher balloon/aorta diameter ratio) and reduces the aortomyoplasty effect, which depends on blood volume changes in the aorta. To address this, Chachques and coworkers assessed the use of a pericardial patch to increase the aortic volume at the aortomyoplasty site using a goat model (4). However, if this is the main reason for the lack of end-diastolic pressure reduction, human clinical aortomyoplasty should render better results. In the current study, the additional component of afterload reduction, namely, the decrease in the next beat's peak systolic pressure, was absent in both the aortomyoplasty and the IABP groups. This was most likely due to the animal model with the small aortic diameter and high aortic compliance, as has been demonstrated in the pediatric population (16). The afterload reduction induced by the IABP may have a negative influence on coronary flow due to the effect of auto regulation.

\subsection{Correlation between hemodynamic variables}

In this study, no correlation was found between peak diastolic pressure augmentation and coronary blood flow augmentation in either the aortomyoplasty or the IABP group. However, there was a strong correlation (Pearson's correlation coefficient $\mathrm{r}^{2}=0.6$ ) between proximal aortic diastolic pressure integral augmentation and coronary blood flow augmentation in the 
IABP. This data has clinical implications and should be addressed when adjusting the IABP setting.

\subsection{Study limitations}

Healthy mongrel dogs served for the animal model used in the current study. Generally, it is difficult to demonstrate in healthy hearts the benefits of innervations conceived to assist the failing heart. However, since the IABP was capable of inducing both diastolic augmentation and afterload reduction in such a healthy animal model, and since this study was designed as a comparison with the IABP, the drawbacks of using a healthy animal model can be considered minimal.

Another limitation of the study is the use of an untrained skeletal muscle. The conditioning protocol results in reduction of muscle power and speed of contraction. However, it may be assumed that a trained muscle would not induce more significant afterload reduction than that induced by an untrained muscle.

An additional problem of the acute experiment may be that the distal, ischemic part of the muscle is not critical in aortomyoplasty studies, since this part of the muscle is not used in the wrapping (17).

\subsection{Conclusions}

In conclusion, afterload reduction achieved by the IABP was not demonstrated during descending aortomyoplasty counterpulsation. However, descending aortomyoplasty induced greater coronary blood flow augmentation than that achieved by the IABP. This may be of significance for end-stage ischemic patients. Long term follow-up in an experimental heart failure model and possible modification of the surgical technique in descending aortomyoplasty are important to clearly study the effects and hemodynamic benefits of this new left ventricular assist system. 


\section{REFERENCES}

1 Fuchs RM, Brin KP, Brinkern JA, Guzman PA, Herzer RR, Yin FCP. Augmentation of regional coronary blood flow by intra-aortic balloon counterpulsation in patients with unstable angina. Circulation 1983;68:117-123.

2 Mundth ED. Mechanical and surgical interventions for the reduction of myocardial ischemia. Circulation 1976;53(3Suppl):I176-83.

3 Freed PS, Wasfie T, Zado B, Kantrowitz A. Intra-aortic balloon pumping for prolonged circulatory support. Am J Cardiol 1988;61:554-557.

4 Chachques JC, Grandjean PA, Fischer EIC, Latremouille C, Jebara VA, Bourgeois I, Carpentier A. Dynamic aortomyoplasty to assist left ventricular failure. Ann Thorac Surg 1990;49:225-230.

5 Chachques JC, Radermercker M, Tolan MJ, Fischer EIC, Grandjean PA, Carpentier A. Aortomyoplasty counterpulsation: Experimental results and early clinical experience. Ann Thorac Surg 1996;61:420-425.

6 Pattison CW, Cumming DVE, Williamson A, Clayton-Jones DG, Dunn MJ, Goldspink G, Yacoub M. Aortic counterpulsation for up to 28 days with autologous latissimus dorsi in sheep. J Thorac Cardiovasc Surg 1991;102:766-773.

7 Lazzara RR, Trumble DR, Magovern JA. Chronic counterpulsation with descending thoracic aortomyoplasty improved cardiac function in animals with heart failure. J Heart Lung Transplant 1994;13:652-660.

8 Chachques JC, Haab F, Cron C, Fischer EIC, Grandjean PA, Bruneval P, Acar C, Jebara VA, Fontaliran F, Carpentier A. Long-term effects of dynamic aortomyoplasty. Ann Thorac Surg 1994;58:128-134.

9 National Academy of Sciences: Guide for the Care and Use of Laboratory Animals. DHHS Publication No. NIH 85-23, revised 1985.

10 Chachques JC, Grandjean PA, Carpentier A. Latissimus dorsi dynamic cardiomyoplasty. Ann Thorac Surg 1989;47:600-604.

11 Bolotin G, Wolf T, Van Der Veen FH, Shofti R, Lorusso R, Schreuder JJ, Uretzky G. Acute descending aortomyoplasty induces coronary blood flow augmentation. Ann Thorac Surg 1999;68:1668-1673.

12 Williams DO, Korr KS, Rewirtz H, Most AS. The effect of intraaortic balloon counterpulsation on regional myocardial blood flow and oxygen consumption in the presence of coronary artery stenosis in patients with unstable angina. Circulation 1982; 66(3):593-7.

13 Angerpointer TA, Jackson PG, Williams BT. The long-term effect of intra-aortic balloon counterpulsation on left ventricular performance. J Cardiovasc Surg (Torino) 1980;21 (4):399-404.

14 Zehetgruber M, Mungigler G, Christ G, Merhaut C, Klaar U, Kratochwill C, Neunteufl T, Hofmann S, Heinz G, Maurer G, Siostrzonek P. Relation of hemodynamic variables to augmentation of left anterior descending coronary flow by intra-aortic balloon pulsation in coronary artery disease. Am J Cardiol 1997;80(7):951-955.

15 Jett GK, Dengle SK, Barnett PA, Platt MR, Willerson JT, Watson JT, Eberhart RC. Intraaortic balloon counterpulsation: its influence alone and combined with various pharmacological agents on regional myocardial blood flow during experimental acute coronary occlusion. Ann Thorac Surg 1981;31 (2):144-154.

16 Veasy LG, Blalock RC, Orth JL, Boucek MM. Intra-aortic balloon in infants and children. Circulation 1983;68(5):1095-1100. 
17 Mesana TG, Mouly-Bandini A, Ferzoco SJ Collard 1: Caus I, Reul RM, Monties JR, Schoen FJ, Cohn LH. Dynamic aortomyoplasty: clinical experience and thoracoscopic surgery feasibility study. J. Card Surg 1998; 13:60-69. 
Chapter

Hemodynamic analysis of descending vs. ascending aortomyoplasty, and comparison with intra-aortic balloon pump

Gil Bolotin, Frederik van der Veen, Robert Shachner, Yuval Sezbon, Daniel Reisfeld, Rona Shofti, Roberto Lorusso, Shlomo Ben-Haim, Gideon Uretzky 


\section{ABSTRACT}

Objective. Descending and ascending aortomyoplasty are two surgical procedures intended to induce hemodynamic benefits similar to those of the intraaortic-balloon-pump (IABP). To date, there have been no studies comparing the two surgical techniques. The objective of this study was to compare coronary blood flow augmentation and afterload reduction as produced by descending and ascending aortomyoplasty counterpulsation.

Methods. Twenty-two mongrel dogs (18-35 kg) underwent IABP application $(n=7)$, descending $(n=8)$, or ascending $(n=7)$ aortomyoplasty. Left anterior descending (LAD) coronary artery blood flow was measured using a Transonic Doppler flow probe. Left ventricular pressure as well as aortic pressures proximal and distal to either the aortomyoplasty site or the IABP position were monitored continuously.

Results. Descending aortomyoplasty induced higher elevation in the LAD blood flow during assisted beats ( $27 \%$ from $10.84 \mathrm{ml} / \mathrm{min}$ to $13.86 \mathrm{ml} / \mathrm{min}$, $\mathrm{P}<0.001)$ than that induced by either ascending aortomyoplasty $(19 \%$ from 11.75 to $145 \mathrm{ml} / \mathrm{min}, \mathrm{P}<0.001$ ) or IABP counterpulsation ( $18 \%$ from 8.63 $\mathrm{ml} / \mathrm{min}$ to $10.2 \pm 4 \mathrm{ml} / \mathrm{min}, \mathrm{P}<0.001$ ). Conversely, while ascending aortomyoplasty reduced the left ventricular end-diastolic pressure by $16 \%$ (from 60 $18 \mathrm{mmHg}$ to $5022 \mathrm{mmHg}, \mathrm{P}<0.001$ ), similar to the $16 \%$ afterload reduction achieved by the IABP counterpulsation, descending aortomyoplasty failed to induce afterload reduction.

Conclusions: Descending aortomyoplasty produces higher coronary blood flow augmentation than either ascending aortomyoplasty or IABP. However, afterload reduction comparable to that achieved by IABP was observed only with ascending aortomyoplasty and not with descending aortomyoplasty.

Key words. Heart Failure, Skeletal Muscle, Aortomyoplasty, IABP 


\section{INTRODUCTION}

Aortomyoplasty is a surgical procedure that aims to induce the hemodynamic effects of the Intra-Aortic Balloon Pump (IABP) (1). However, while IABP is used for acute situations and is limited to no more than several days of treatment, aortomyoplasty may serve as a chronic treatment for years (2). Two different surgical configurations have been reported for aortomyoplasty: ascending aortomyoplasty (the right latissimus dorsi muscle wrapped around the ascending aorta) and descending aortomyoplasty (the left latissimus dorsi muscle wrapped around the descending aorta) $(3,4)$. Both surgical techniques were experimentally evaluated and both were performed clinically $(5,6)$. However, there is no data in the literature concerning the comparison between ascending and descending aortomyoplasty. The objective of this study was to compare the hemodynamic effects of these two techniques and to compare them to the effects of the IABP.

\section{METHODS}

Twenty two mongrel dogs weighing $18-35 \mathrm{~kg}$ were used for this study. Seven underwent acute ascending aortomyoplasty, eight underwent acute descending aortomyoplasty, and seven had IABP application. The experiments were performed in accordance with the "Guide for the Care and Use of Laboratory Animals" (7).

All animals underwent the same general anesthesia induced by i.v. sodium thiopental (Penthotal) $15 \mathrm{mg} / \mathrm{kg}$, and maintained following endotrachial intubation with $\mathrm{O}_{2}$ : $\mathrm{NO}(1: 2)$ and $1.5 \%$ Fluothane. Throughout the experiments, lung ventilation was achieved using a positive pressure respirator (Harvard). Body temperature was kept constant using a heating mattress. Prior to surgery, a single dose of $5000 \mathrm{U}$ i.v. heparin was administered.

\section{Ascending aortomyoplasty: surgical technique}

A right-sided midaxillary incision was performed above the right LD muscle and all collateral blood vessels to the distal part of the muscle were ligated or coagulated. All attachments of the muscle, except for the axillary pedicle, were disconnected, in order to keep the thoracodorsal artery, vein, and nerve intact. Two intramuscular electrodes (Medtronic SP 5590 stimulation leads) were implanted in the upper part of the LD muscle flap, perpendicular to the main branches of the thoracodorsal nerve, as described previously by Chachques and coworkers (8). To ensure proper positioning of the electrodes, satisfactory threshold $(0.3-0.6 \mathrm{~V})$ and total recruitment $(1.0-2.5 \mathrm{~V})$ values were 
obtained following comnection of the electrodes to the stimulator system (Medtronic Cardio-Myo Stimulator SP 3076). A $5 \mathrm{~cm}$ segment of the anterior portion of the right second $\mathrm{rib}$, including the periosteum, was then resected to allow transposition of the LD muscle flap into the thorax. The muscle was inserted into the chest cavity, its tendon cut and sutured to the periosteum of the third rib, prior to closing the thoracic window.

The skin incision was then sutured and the animal position was changed to allow a median sternotomy. After the median sternotomy, the pericard was opened and a sensing electrode (Medtronic 6500 sensing lead) was implanted in the right ventricular wall (adjacent to the septum) and the sensing threshold (4.5-16.4 $\mathrm{mV}$ ) was measured. To enlarge the portion of the ascending aorta to be wrapped, a dissection was performed between the aorta and the pulmonary artery. Another portion of the ascending aorta was exposed by dissecting toward the aortic annulus. The right latissimus dorsi muscle was then wrapped around the ascending aorta in a single layer (clockwise) and was fixed using prolene $2 / 0$ interrupted sutures.

\section{Descending aortomyoplasty: surgical technique}

The left latissimus dorsi was dissected and introduced into the left chest using the same technique described above for the right latissimus dorsi during ascending aortomyoplasty. Through the same skin incision the thorax was then opened at the fourth left intercostal space and the pericardium cut open. A sensing electrode (Medtronic 6500 sensing lead) was implanted in the right ventricular wall (adjacent to the septum) and the sensing threshold (4.5-16.4 $\mathrm{mV}$ ) was measured. A segment of approximately $8-10 \mathrm{~cm}$ of the descending aorta (1.5-2.5 $\mathrm{cm}$ in diameter), distal to the left subclavian artery, was then mobilized. All intercostal arteries arising from the aorta in this particular portion were ligated and divided. The left LD muscle flap was subsequently wrapped around the exposed descending aorta (single layer) using Prolene $2 / 0$ sutures. The configuration required tight wrapping of the latissimus dorsi around the aorta in a counter-clockwise orientation (best configuration from previous study (9).

\section{LD Muscle Stimulation and IABP Setting}

Stimulation commenced immediately following application of each surgical configuration. The first part of this protocol comprised a six-pulse burst (5 volt, $165 \mu \mathrm{sec}$ pulse width, $200 \mathrm{msec}$ burst duration) using different delays $(150,200,250,300,350,400$ or $450 \mathrm{msec})$ after the QRS complex, in order to 
cover the entire diastolic range. The stimulation burst was applied every third or fourth spontaneous heartbeat.

The IABP was inserted through the femoral artery and its exact position was determined using fluoroscopic guidance. The IABP was activated in a $1: 3$ ratio, to allow comparison between the assisted beats and the previous, unassisted beats. The balloon was inflated at different delays after the QRS complex (from 200 to $400 \mathrm{msec}$ ) and with varying inflation duration (from 150 to $250 \mathrm{msec}$ ). The best delay and duration were explored by increments of $50 \mathrm{msec}$ and the decision was made according to the plotted proximal blood pressure curve.

\section{Instrumentation and Data Collection}

A Millar (Millar Instruments, Texas, USA) solid state pressure catheter was inserted via the left carotid artery and advanced into the left ventricle (LV). Two additional solid state pressure catheters were inserted via the right and left femoral arteries and advanced into the aorta, proximally and distally to the wrapping site, respectively.

A Doppler flow probe (Transonic Systems, NY) was placed around the left anterior descending (LAD) coronary artery, proximal to the first diagonal, for measurement of coronary blood flow. Surface ECG was monitored continuously throughout the experiments.

\section{Data and Statistical Analysis}

Hemodynamic data was gathered on an IBM personal computer using CODAS (DATAQ, Akron, $\mathrm{OH}$ ) and HDAS (Maastricht, The Netherlands) software. Additional software was designed to detect peak diastolic pressure and coronary blood flow and to calculate diastolic pressure and coronary blood flow curve integral during the diastolic phase (area under the curve). Assisted beats were compared to prior, unassisted beats using Student's paired t test. Results are expressed as mean $\pm S D$. Differences were considered significant at a $\mathrm{P}$ value $<0.05$.

\section{RESULTS}

\section{Surgical technical considerations}

The mean descending aortic diameter was $19 \pm 3 \mathrm{~mm}$ and the mean length of the aortic segment to be wrapped was $92 \pm 6 \mathrm{~mm}$, resulting in $26 \pm 2 \mathrm{ml}$ volume of wrapped descending aorta. The mean ascending aortic diameter was $23 \pm 2$ 
$\mathrm{mm}$ and the mean length of arta to be wrapped was $46 \pm 1 \mathrm{~nm}$, resulting in 19 $\pm 2 \mathrm{ml}$ volume of wrapped ascending aorta $(\mathrm{p}<0.00 \mathrm{l}$ between the wrapped volumes\}.

Descending aortomyoplasty was found to be a simpler operation compared to ascending aortomyoplasty, performed through one skin incision without the need to re-position the animal on the operating table or to open the pericardium. All descending aortomyoplasty animals survived the entire experimental protocol. Ascending aortomyoplasty was found to be a more demanding operation, done through a right thoracic skin incision followed by mid sternotomy, which necessitates changing the animal's position in the middle of the operation. The main technical problem in the ascending aortomyoplasty operation was the short segment of the ascending aorta before the first branch, which required a wide dissection around the ascending aorta toward the heart, as well as the dissection of parts of the latissimus dorsi muscle in three of the eight experiments, in order to facilitate the wrapping of the muscle around the ascending aorta. This dissection caused both bleeding from the adjacent tissue as well as supra ventricular arrhythmias. Two animals died during the wrapping stage (and were excluded from the study results) due to major bleeding combined with arrhythmias.

\section{Timing of the counterpulsation}

Both the contraction of the LD muscle around the descending or ascending aorta and the IABP counterpulsation induced diastolic pressure augmentation in the proximal aorta. However, exact timing was crucial for achieving the desired hemodynamic benefits. Shorter delays (150 ms following the QRS complex) induced aortic regurgitation before aortic valve closure, identified by the pressure sensor in the LV. Moreover, the shorter delays induced higher proximal aortic pressure during the last part of the systole, resembling aortic narrowing, an effect contrary to the desired afterload reduction. Counterpulsation during the latter part of the ventricular diastole $(450 \mathrm{msec}$ following the QRS complex) resulted in a less drastic diastolic pressure augmentation. As found with the shorts delays, this long delay caused partial obstruction of the aorta during the next systole.

\section{Hemodynamic parameters}

Comparison of the diastolic augmentation achieved by the surgical configuration reveals an advantage to the descending technique in the coronary blood flow during the assisted beats as compared to the unassisted beats (Figure 1). While ascending aortomyoplasty generated coronary blood flow augmenta- 
tion similar to that achieved by the IABP (19\% and 18\%), descending aortomyoplasty induced a $27 \%$ increase in the coronary flow during the assisted beats.

These changes in coronary blood flow were found to be correlated with the proximal aortic diastolic pressure, and again significantly higher augmentation was attained in the descending aortomyoplasty group (Table 1).

Proximal aortic end-diastolic pressure reduction resembling afterload reduction was consistently achieved in the assisted beats during the ascending aortomyoplasty operations, comparable to the reduction found in the IABP group (Figure 2). In contrast, descending aortomyoplasty failed to induce receptive proximal aortic end-diastolic pressure reduction.

\section{DISCUSSION}

Ascending and descending aortomyoplasty have both been evaluated experimentally and at the early clinical stage. In ascending aortomyoplasty the right latissimus dorsi was harvested through a right thoracic incision, and then wrapped around the ascending aorta, which was reached through a midsternotomy (3). Descending aortomyoplasty as presented by Pattison and coworkers was performed through a single left thoracic incision using the left latissimus dorsi, which was then wrapped around the descending aorta (4). These surgical differences result in major implications for both the surgical technique and its hemodynamic effects.

\section{Surgical technical considerations}

As reported by Mesana and coworkers, in the current study we found the descending aortomyoplasty to be a faster and easier procedure due to the single skin incision, the single position of the surgical candidate throughout the entire operation, and the fact that the pericardium could remain unopened (6). The avoidance of pericardial opening may be even more important when performing the procedure following a previous cardiac operation.

Another difference which exists between the two techniques is the size and the structure of the descending and ascending aortas. As in humans, the ascending aortic diameter was found to be larger than the descending aortic diameter (the hemodynamic implications of which will be discussed later); however, it was the short length of the ascending aorta before the first branch to be wrapped that caused surgical difficulties. In three of the experiments, parts of the latissimus dorsi muscle had to be removed to facilitate the wrapping, a procedure that may cause long-term damage to the muscle. However, 


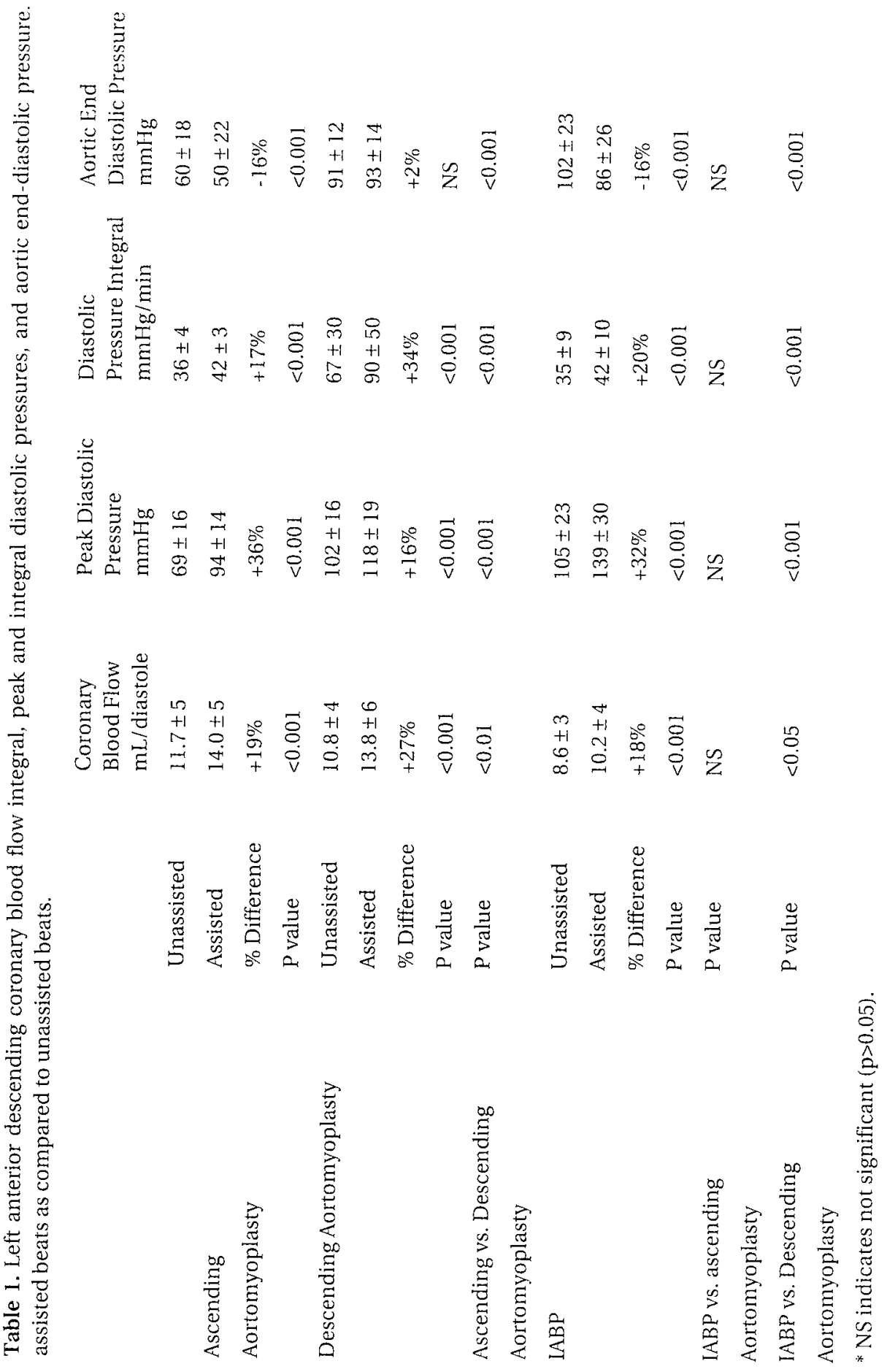




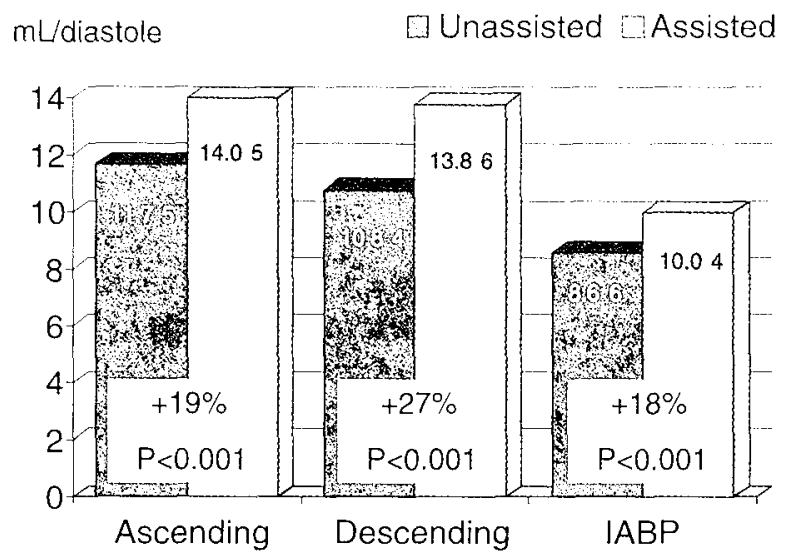

Figure 1. Coronary blood flow (area under the curve mL/diastole), in assisted beats as compared to unassisted beats during IABP, ascending and descending aortomyoplasty counterpulsation.
$\mathrm{mmHg}$
圈 Unassisted $\square$ Assisted

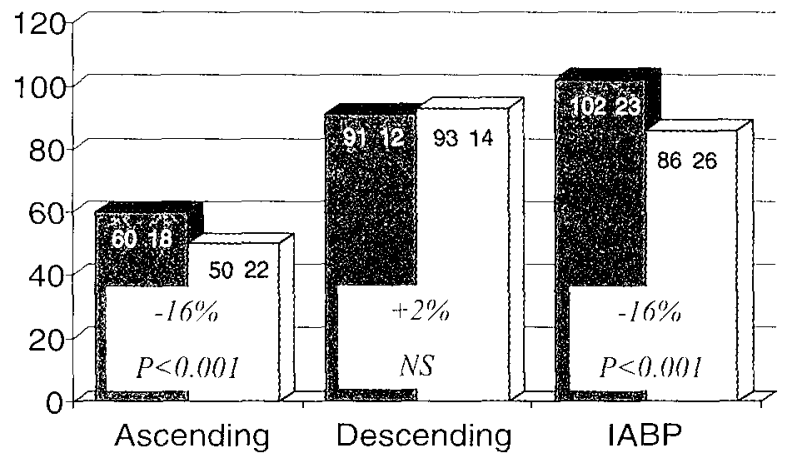

Figure 2. Proximal aortic end-diastolic pressure, in assisted beats as compared to unassisted beats during IABP, ascending and descending aortomyoplasty counterpulsation.

in the last six ascending aortomyoplasty experiments, a wide dissection around the aorta toward the aortic root enabled the wrapping to be completed without cutting of the skeletal muscle, but with more bleeding and supraventricular arrhythmias. The death of two animals during this stage was probably related to the learning curve needed in this part of the operation. Cabrera and coworkers described ascending aortomyoplasty with the left latissimus dorsi without any technical problems during the wrapping (10). This is most 
probably due to the long segment of the ascending aorta before the first branch in sheep as compared to the animal model used in the current study. Moreover, looking at the different anatomy of the ascending aorta in humans, it is safe to assume that both the diameter and the length of the aorta to be wrapped will be larger in clinical situations.

Descending aortomyoplasty usually requires the ligation of one or more intercostal arteries, with the subsequent potential complication of postoperative neurological disorders (Adamkiewitz artery). Cernaianu reported a modification of the descending aortomyoplasty operation in an attempt to preserve the intercostal arteries (11). Indeed, the use of several muscle splittings at the level of the intercostal artery and of pericardial "bridges" to posteriorly connect the LDM to the wrapped aortic portion has been shown previously. Another clinical issue that should be taken into consideration when comparing the two procedures is the relatively higher prevalence of atherosclerosis in the descending aorta as compared to the ascending aorta (12).

In general, we found the descending aortomyoplasty to be a more simple procedure. These differences may become less important once more experience with the two techniques has been gathered; however, since patients who are candidates for aortomyoplasty are most often at high surgical risk, the implementation of a less invasive procedure may be important (2).

\section{Timing of the counterpulsation}

The timing of skeletal muscle contraction around the descending aorta is as crucial as the timing of the IABP counterpulsation $(13,14)$. Zelano and coworkers have demonstrated that increased afterload was due to an inappropriate setting of the IABP, a finding that our previous work replicated with regard to descending aortomyoplasty $(9,15)$. An increased afterload in hemodynamically unstable patients may have detrimental effects. To prevent a possible increase in afterload in certain clinical situations in patients after aortomyoplasty, two issues should be addressed: 1) the chronic decrease in muscle power and speed of contraction; and 2) changes in the patient's heart rate and general hemodynamic situation. Both issues will probably demand frequent clinical evaluation of the patient and modification of stimulation settings.

\section{Hemodynamic parameters}

Descending aortomyoplasty was found to be superior in coronary blood flow augmentation. This may be due to the higher aortic volume to be wrapped in this procedure $(26 \pm 2 \mathrm{ml}$ as compared to $19 \pm 2 \mathrm{ml}$ in the ascending 
aortomyoplasty). However, since the augmentation was found to be higher than in the IABP control group (using a $30 \mathrm{ml}$ pediatric balloon) another explanation should be proposed. A possible explanation is the orientation of the skeletal muscle fibers. In the case of descending aortomyoplasty, the contraction upward induces squeezing of the aorta proximally, and thus may increase the diastolic augmentation. In ascending aortomyoplasty, the orientation causes squeezing of the aorta distally (although to a lesser extent), which may reduce diastolic augmentation. Cabrera and coworkers report a hemodynamic advantage of ascending aortomyoplasty over abdominal descending aortomyoplasty (10). However, the use of the abdominal thoracic aorta may result in less prominent augmentation compared to that achieved by wrapping the thoracic descending aorta, as in the current study. The proximal aortic end-diastolic pressure reduction induced by ascending aortomyoplasty was equal to that achieved with the IABP, constituting an important advantage over descending aortomyoplasty. The reasons for that effect may be the proximity of the ascending aorta to the coronary ostea, its larger diameter, which results in higher volume changes due to diameter changes and perhaps differences in aortic wall elasticity. These results suggest the superiority of ascending aortomyoplasty for end-stage heart failure patients, whereas descending aortomyoplasty may be more suitable for end-stage ischemic patients. Cabrera and coworkers did report important hemodynamic effects of ascending versus descending aortomyoplasty; however, their hemodynamic data did not include coronary flow and afterload reduction (10). Other studies showed important hemodynamic benefits of ascending or descending aortomyoplasty, but since all studies were done using only one of the techniques, no objective comparison between the two is possible $(16,17)$.

\section{Theoretical clinical advantages of aortomyoplasty}

The chronic hemodynamic effects of descending aortomyoplasty present several possible advantages for ischemic patients. It is possible to increase the efficacy of aortomyoplasty according to the clinical demands of the patient by changing the amplitude and the rate (telemetric setting changes) of the latissimus dorsi muscle contraction. Lowering the stimulation amplitude and, more importantly, reducing the stimulation/spontaneous beat ratio can protect the muscle from chronic damage while still maintaining some of its protective effects. Once unstable angina or even acute myocardial infarction does occur, increasing aortomyoplasty efficacy may attenuate the angina and reduce infarct size, as has previously been demonstrated with the IABP (18). Utilization of the IABP is beneficial in acute situations, such as cardiogenic shock, cardio-pulmonary resuscitation, and as an adjuvant treatment to 
thrombolytic therapy $(19,20)$. Similarly, exicerbation in a patient's clinical status, even years after aortomyoplasty, may he treated by increasing both the latissimus dorsi muscle power and the ratio of assisted contractions to spontaneous beats.

In conclusion, both surgical techniques were found to generate hemodynamic effects comparable to those of the IABP. However, descending aortomyoplasty was found to be a more simple operation, capable of inducing higher coronary flow augmentation; thus, it may be particularly helpful for treating end-stage ischemic patients. Ascending aortomyoplasty was found to produce hemodynamic effects similar to those of IABP, including afterload reduction, and may be more suitable for treating end-stage heart failure patients.

\section{Acknowledgements}

This study was supported (in part) by grant no. 3737 from the Chief Scientist's Office of the Ministry of Health, Israel. 


\section{REFERENCES}

1 Lazzara RR, Trumble DR, Magovern IA. Dynamic descending thoracic aortomyoplasty: comparison with intraaortic balloon pump in a model of heart failure. $\Lambda$ nn Thorac Surg $1994 ; 58(2): 366-370$.

2 Chachques JC, Radermercker M, Tolan MJ, Fischer II, Grandjean P $\Lambda$, Carpentier $\Lambda$. Aortomyoplasty counterpulsation: experimental results and early clinical experience. Ann Thorac Surg 1996;61 (1):420-425.

3 Chachques JC, Grandjean PA, Fischer III, Latremouille C, Jebara VA, Bourgeois I, Carpentier $A$. Dynamic aortomyoplasty to assist left ventricular failure. $\Lambda$ nn Thorac Surg 1990;49(2):225-230.

4 Pattison CW, Cumming DV, Williamson A, Clayton-Jones DG, Dunn MJ, Goldspink (i, Yacoub M. Aortic counterpulsation for up to 28 days with autologous latissimus dorsi in sheep. J Thorac Cardiovasc Surg 1991; 102(5):766-773.

5 Trainini J, Barisani JC, Cabrera Fischer I:I, Chada S, Christen AI, Elencwajg B. Chronic aortic counterpulsation with latissimus dorsi in heart failure: clinical follow-up. J heart Lung Transplant. 1999;18(11):1120-1125.

6 Mesana TG, Mouly-Bandini A, Ferzoco SJ, Collart F, Caus T, Reul RM, Monties JR, Schoen FJ, Cohn LH. Dynamic aortomyoplasty: clinical experience and thoracoscopic surgery feasibility study. J Card Surg 1998;13(1):60-69.

7 National Academy of Sciences: Guide for the Care and Use of Laboratory Animals. DHHS Publication No. NII 85-23, revised 1985.

8 Chachques JC, Grandjean PA, Carpentier A. Latissimus dorsi dynamic Cardiomyoplasty. Ann Thorac Surg 1989;47: 600-604.

9 Bolotin G, Wolf T, Van Der Veen FH, Shofti R, Lorusso R, Shreuder JJ, Uretzky G. Acute descending aortomyoplasty induces coronary blood flow augmentation. Ann Thorac Surg 1999;68: 1668-1673.

10 Cabrera EI, Christen AI, de Forteza E, Risk MR. Dynamic abdominal and thoracic aortomyoplasty in heart failure: assessment of counterpulsation. Ann Thorac Surg 1999;67:1022-1029.

I 1 Flum DR, Cernaianu AC, Meada R, Lee L $\Lambda$, Salartash K, Grosso M $\Lambda$, Weiss RL, Cilley JII, Delrossi AJ. Descending thoracic aortomyoplasty: a technique for clinical application. Ann Thorac Surg 1996; 61: 93-98.

12 Nishino M, Masugata H, Yamada Y, Abe H, Hori M, Kamada T. Evaluation of thoracic aorta atherosclerosis by transesophageal echocardiography. Am Heart J 1994;127(2): 336-344.

13 Hakami A, Santamore WP, Stermel RW, Tobin G, Hjortdal VE. Evaluation of stimulation parameters on aortomyoplasty, using Latissimus Dorsi muscle in a goat model: an acute study. Eur J Cardiothorac Surg 1999;16(2):228-232.

14 Zelano JA, Li JK, Weilkowitz W. A closed-loop control scheme for intraaortic balloon pumping. IEEE Trans Biomed Eng 1990;37(2):182-192.

15 Zelano JA, Ko W, Lazzaro R, et al. Comparison of an extraaortic counterpulsation device versus intraaortic balloon pumping in severe cardiac failure. ASAIO Trans 1991;37(3): M342-4.

16 Cardone JC, Yoon PD, Trumble DR, Magovern JA. Regional effects of aortomyoplasty in acute ischemia. Ann Thorac Surg 1996;61:426-429. 
17 Chachques JC, Haab F, Cron C, lischer EC, Grandjean PA, Bruneval P, $A$ aar $C$, Jebara VA, Fontaliran F, MJ, Carpentier $A$. Long-term effects of dynamic aortomyoplasty. Ann Thorac Surg 1994;58:128-134.

18 Goldfarb D, Freisinger GC, Conti CR, Brown BG, Gott VL. Preservation of myocardial viability by diastolic augmentation after ligation of coronary artery in dogs. Surgery 1968;63:320-327.

19 Gurbel PA, Anderson RD, MacCord CS, et al. Arterial diastolic pressure augmentation by intra-aortic balloon counterpulsation enhances the onset of coronary artery reperfusion by thrombolytic therapy. Circulation 1994;89(1):361-365.

20 Emerman CL, Pinchak AC, Hagen JI, Hancock D. Hemodynamic effects of the intraaortic balloon pump during experimental cardiac arrest. Am J Imerg Med 1989;7(4): 378-383. 
Chapter

Summary and conclusions 
This thesis can be summarized and concluded into four categories:

1. The Carotid-Jugular $A-V$ shunt model to induce ventricular dilatation

2. The Cardionyoplasty procedure

3. The Aortomyoplasty procedure

4. Cardiac monitoring and imaging modalities for preclinical studies

\section{Chapter 2 - The Carotid-Jugular A-V shunt model}

This study demonstrates that the A-V shunt heart failure model is capable of producing stable, immediate and long-term high cardiac output (overload) dilatation. The successful compensatory mechanism in the goat carotid jugular A-V shunt is the reason for the lack of signs of renal failure as demonstrated in a rat aortovenocaval fistula by Wegner and coworkers (1). Therefore, the AV shunt model described should be considered as a compensatory overload dilatation model rather than a heart failure model.

The increase in cardiac output by about $100 \%$ generated huge hemodynamic changes. The LVEDV increased while the LVESV decreased, resulting in an abrupt rise in left ventricular ejection fraction and stroke volume. Surprisingly, no changes in heart rate were observed. These changes were reversible at this point in time as observed by closure of the shunt. These findings suggest that the extensive changes observed in left ventricular dimensions are within the efficient part on Starling's curve. Echocardiography tests revealed a gradual increase in left ventricular end-diastolic diameter, reaching maximal left ventricular dilatation at 8 weeks. These findings were confirmed in the final experiments using the conductance catheter technique. The practical advantage of the shunt model is that it is possible to evaluate the patency of the shunt by palpating the blood flow thrill in the neck. The shunt's location facilitates access to ultrasonic measures for a more precise evaluation, as well as temporary closure, obtained by applying manual pressure on the animal's neck.

In conclusion, the carotid jugular $\mathrm{A}-\mathrm{V}$ shunt in goats causes significant left heart dilatation without signs of heart failure, and is reproducible with low animal mortality.

\section{Cardiomyoplasty}

The wrapping procedure, which was monitored in Chapter 3, induced a major reduction in both cardiac output and left ventricular volumes, followed by partial recovery 45 minutes later. These results are supported by clinical reports of acute deterioration in the immediate postoperative period for 
which Carpentier and Mesana proposed extensive use of IABP and inotropic support both before and after the operation $(2,3)$.

After introducing the LD into the thorax through the intercostal window both cardiac output and left ventricular end-diastolic volume (LVEDV) decreased the wrapping slightly, yet to a statistically significant degree, acute reduction in both cardiac output and LVEDV. These experimental results are supported by the clinical data published by Barbier and coworkers, demonstrating acute modification of left and right ventricular geometry monitored by echocardiography immediately after cardiomyoplasty (4). These results revealed another risk of cardiomyoplasty, and might explain part of the perioperative mortality, in particular in patients with very dilated and poor left ventricular function who might lack the reserve to overcome this dangerous phase in the procedure (5). The partial recovery within 45 minutes in both cardiac output (86\%) and LVEDV (88\%) appears relatively fast and is most likely due to the fact that this is a compensatory and not a failing, heart model.

Chapter 4 describes a study in which we deliberately chose to start LD muscle stimulation immediately after the cardiomyoplasty procedure, hypothesizing that ventricular dilatation and the state of the LD muscle is the key to obtaining systolic augmentation. With the proper skeletal muscle stimulation setting, significant systolic augmentation was demonstrated. These results emphasize the importance of heart dilatation in inducing pronounced systolic augmentation and the value of precise tailoring of the individual stimulation setting to achieve optimal effects and avoid interference with the next heart beat.

In Chapter 5 the novel three-dimensional electroanatomical mapping system was used to obtain a more detailed understanding of the 3D geometric changes induced by both the wrapping of the skeletal muscle around the heart and the stimulation protocol. Data from the cross section areas indicate that the reduction in the left-ventricular end-diastolic volume after the wrapping is mainly in the mid and base part of the heart.

During the stimulation protocol, change in the long axis of the left ventricle in the assisted beats was documented, in addition to changes in local point movements in different parts of the heart as a result of contraction of the skeletal muscle around the left ventricle. The three cross-section areas of the left ventricle during the normal cardiac cycle and the assisted beats supply us with new data regarding the squeezing of the left ventricle by the skeletal muscle. From the clear reduction noted in cross-area of the mid part of the heart during the assisted systole, we can conclude that this is the part of the heart that is most affected by cardiomyoplasty. The three-dimensional electroanatomical mapping system allows detailed reconstruction of the left ventricular geometry and affords us a clear view of the difference between the 
assisted and the unassisted beats. This novel monitoring system should serve as an important tool for the analysis and development of new techniques in cardiac surgery.

\section{Aortomyoplasty}

In the study reviewed in Chapter 6, acute descending aortomyoplasty was shown to induce both proximal diastolic pressure and coronary blood flow augmentation. Optimal timing of muscle stimulation was found to be important for achieving the best assist. The diversity of results obtained when applying different surgical configurations as well as the occasional demonstration of afterload reduction emphasize the need for further investigation and indicate the potential benefit of determining a chronic setting for the trained skeletal muscle, so as to optimize this surgical modality.

In Chapter 7 we compared the hemodynamic effects of descending aortomyoplasty with the current gold standard, IABP counterpulsation. The afterload reduction achieved by the IABP was not demonstrated during descending aortomyoplasty counterpulsation; however, descending aortomyoplasty did induce greater coronary blood flow augmentation than that achieved by the IABP. This finding may be of significance for end-stage ischemic patients.

Two different surgical configurations have been reported for aortomyoplasty: ascending aortomyoplasty (the right latissimus dorsi muscle wrapped around the ascending aorta) and descending aortomyoplasty (the left latissimus dorsi muscle wrapped around the descending aorta) $(6,7)$. Both surgical techniques were experimentally evaluated and both were performed clinically $(8,9)$. However, there is no data in the literature concerning the comparison between ascending and descending aortomyoplasty. The objective in Chapter $\mathbf{8}$ was to compare the hemodynamic effects of these two techniques as well as to compare them with the hemodynamic effects induced by the IABP. Descending aortomyoplasty produces higher coronary blood flow augmentation than either ascending aortomyoplasty or IABP. This procedure, therefore, may be more appropriate for end stage ischemic patients. However, afterload reduction, comparable to that achieved by IABP, was observed only with ascending aortomyoplasty and not with descending aortomyoplasty. According to our results, ascending aortomyoplasty should be applied to end stage heart failure patients. 


\section{Cardiac monitoring and imaging modalities - chapter 9}

In this thesis, several important cardiac imaging and monitoring technologies were used. To evaluate left ventricular hemodynamics and geometrics, the conductance catheter technique and the three-dimensional electroanatomical mapping technique. Both techniques were proven to be highly accurate when compared to other modalities $(10,11,12)$. The conductance catheter technique is capable of generating a beat-to-beat real time pressure volume loop and is highly sensitive to minor hemodynamic differences between the beats (13). This is an important feature for the understanding and analysis of cardiac intervention that does not assist every spontaneous beat, as in the case of the IABP, certain assist devices, cardiac pacing, and operations such as aortomyoplasty and cardiomyoplasty. The main drawback of the conductance catheter technology is the inability of the system to evaluate hemodynamic changes in different regions within the left ventricle. The advantage of the electroanatomic mapping technique over the conductance catheter technique is its capability to demonstrate regional changes within the left ventricle (14).

The ability of the electroanatomic mapping system to identify and label particular regions and reconstruct the $3 \mathrm{D}$ beating left ventricle enabled a beatto-beat analysis of the mean map gathered over a period of 20-30 minutes. Therefore, this system is less sensitive to differences between beats than is the conductance catheter technique, and more vulnerable to animal or patient instability. Its ability to sense and present the endocardial activation propagation may be important for the analysis of surgery that interferes with the endocardial integrity, such as the Batista operation and aneurysmectomy procedures.

In conclusion, echocardiography is a useful tool for general monitoring of animals during chronic experiments, reducing unnecessary invasive tests. However, once measurement of precise, objective hemodynamic data is required, this technology, at the moment, is neither objective nor precise enough. The Swan-Ganz catheter, the arterial blood pressure catheters, and coronary blood flow probes are well proven to be accurate and reliable methods and should be used according to the specific needs and aims of the particular study. Once more details regarding the hemodynamics and geometrics of the heart are needed, the conductance catheter and electroanatomic mapping techniques are both excellent options, and selection between the two should be based on the relative advantages of each, as summarized in the present chapter. 


\section{REFERENCES}

1 Wegner M. Hirth-Dietrich C, Stasch JP. Role of neutral endopeptidase 24.11 in $\Lambda \mathrm{V}$ fistular rat model of heart failure. Cardiovasc Res 1996;31 (6):891-898.

2 Carpentier A, Chachques JC, Acar C, Relland J, Mihaileanu S, Bensasson D, Kieffer JP, Giubourt P, Tournay D, Roussin I, Grandjean PA. Dynamic cardiomyoplasty at seven years. J Thorac Cardiovasc Surg 1993; 106:42-53.

3 Mesana TG, Bauer S, Caus T, Pomane C, Mouly $\Lambda$, Monties JR. Circulatory assist techniques after cardiomyoplasty: determinants for clinical outcome and later consequences. ASAIO J 1995;41:M469-M472.

4 Barbier P. Gerometta P. Tamborini G. Biglioli P. Sisillo l: Guazzi MD. Acute effects of dynamic cardiomyoplasty on ventricular geometry and left ventricular filling detected by transesophageal doppler echocardiography. $\mathrm{Am}$ J Cardiol 1996; 77(9); 783-787.

5 Grandjean PA, Austin L, Chan S, Terpstra B, Bourgeois IM. Dynamic cardiomyoplasty: Clinical follow-up results. J Cardiac Surg 1991;6(suppl): 80-88.

6 Chachques JC, Grandjean PA, Fischer EI, Latremouille C, Jebara VA, Bourgeois I, Carpentier A. Dynamic aortomyoplasty to assist left ventricular failure. Ann Thorac Surg 1990;49(2):225-230.

7 Pattison CW, Cumming DV, Williamson A, Clayton-Jones DG, Dumn MJ, Goldspink G, Yacoub M. Aortic counterpulsation for up to 28 days with autologous latissimus dorsi in sheep. J Thorac Cardiovasc Surg 1991;102(5):766-773.

8 Trainini J, Barisani JC, Cabrera Fischer LI, Chada S, Christen $\Lambda \mathrm{I}$, Elencwajg B. Chronic aortic counterpulsation with latissimus dorsi in heart failure: clinical follow-up. J Heart Lung Transplant. 1999;18(11):1120-1125.

9 Mesana TG, Mouly-Bandini $\Lambda$, Ferzoco SJ, Collart F, Caus T, Reul RM, Monties JR, Schoen FJ, Cohn LH. Dynamic aortomyoplasty: clinical experience and thoracoscopic surgery feasibility study. J Card Surg 1998;13(1):60-69.

10 Burkhoff ZD, Van der Valde E, Kass D, Baan J, Maughan W, Sagawa K. Accuracy of volume measurements by conductance catheter in isolated, ejecting canine hearts. Circulation 1985;72(2):440-447.

11 Gepstein L, Hayam G, Ben-Haim S $\Lambda$. $\Lambda$ novel method for nonfluoroscopic catheterbased electroanatomical mapping of the heart. In vitro and in vivo accuracy results. Circulation 1997;95:1611-1622.

12 Gepstein L, Hayam G, Shpun S, Ben-Haim SA. Hemodynamic evaluation of heart with a nonfluoroscopic electroanatomical mapping technique. Circulation 1997;96:3672- 3680.

13 Dritsas A, Joshi J, Webb SC, Athanassopoulos G, Oakley CM, Nihoyannopoulos P. Beatto beat variability in stroke volume during VVI pacing as predictor of hemodynamic benefit from DDD pacing. PACE 1993;16:1713-1718.

I4 Gepstein L, Goldin A, Lessick J, Hayam G, Shpun S, Schwartz Y, Hakim G, Shofti R, Turgeman A, Kirshenbaum D, Ben-Haim SA. Electromechanical characterization of chronic myocardial infarction in the canine coronary occlusion model. Circulation 1998;98:2055-2064. 
Chapter

Samenvatting en conclusies 
Hoofdstuk 1 geeft een inleiding over de ziekte hartfalen en de chirurgische mogelijkheden om patiënten met ernstig hartfalen te behandelen. Ondanks verbeterde medicatie therapie van patiënten met hartfalen, kent deze ziekte nog steeds een hoge morbiditeit en mortaliteit in de Westerse Wereld. Momenteel maakt een optimale medicamenteuze behandeling gebruik van ACE-remmers, bètablokkers, amiodarone en inotropica, maar desondanks is in 2000 de sterfte van patiënten met ernstig hartfalen nog meer dan $10 \%$ per jaar.

De met medicijnen uitbehandelde hartfaal patiënten, in NYHA klasse III en IV, kunnen alsnog chirurgisch worden behandeld. Als ischemie de ziekteoorzaak is, wordt CABG (aorto-coronaire bypass chirurgie) toegepast die leidt tot verbetering in hartfunctie, of alleen tot vermindering van ziekteverschijnselen en sterftekans.

Naast CABG is harttransplantatie nu een standaardingreep geworden, die helaas slechts bij een beperkt aantal patiënten kan worden uitgevoerd door een tekort aan donorharten. Terwijl in 2000 ongeveer 50 harttransplantaties plaatsvonden in Nederland, zou op basis van recente berekeningen voor 2500 patiënten een harttransplantatie noodzakelijk zijn.

Toepassing van linker ventrikel assist devices (LVAD's of kunstharten) vindt op dit moment alleen plaats bij patiënten als overbrugging naar de harttransplantatie. Deze toepassing zou in de toekomst kunnen worden uitgebreid als deze LVAD's enkele dagen tot weken worden aangesloten om het hart te laten herstellen, of als de LVAD's in staat blijken om permanent het hart te ondersteunen of zelfs totaal te vervangen.

Voorlopig bestaat er een behoefte om het hart langere tijd te kunnen ondersteunen, vooral als een harttransplantatie is uitgesloten. Zowel cardiomyoplastie als aortamyoplastie zijn chirurgische ingrepen, waarbij een rugspier (de latissimus dorsi) wordt gebruikt om de hartfunctie te verbeteren. Dit proefschrift richt zich op deze twee ingrepen.

Het idee om een skeletspier te gebruiken als energiebron om de hartfunctie te verbeteren, is al tientallen jaren oud, maar pas in 1985 is de eerste patiënt behandeld met een cardiomyoplastie operatie. De rugspier werd hierbij om het hart gewikkeld en vastgezet. Deze spier kon vervolgens synchroon met de hartspier samentrekken door gebruik te maken van een speciale spierstimulator.

Een belangrijke doorbraak in de toepassing van deze techniek was de ontdekking enkele jaren tevoren, dat deze rugspier door training in enkele weken was om te vormen tot een vrijwel onvermoeibare spier.

Bij de aortamyoplastie operatie wordt dezelfde rugspier gebruikt om rond de aorta te wikkelen. Ook hier kan de spier synchroon met de hartspier sa- 
mentrekken. De functie komt overeen met die van een intra aorta ballonpomp (IABP), maar is bedoeld als permanente ondersteuning.

Toepassing van een lichaamseigen spier kan veel voordelen hebben boven een harttransplantatie of een LVAD. Afweerreacties zijn niet te verwachten, ontstolling is niet nodig en er zijn geen verbindingen nodig door de huid naar een externe, vaak omvangrijke energiebron en regelmechaniek.

De hoofdstukken 2, 3, 4 en 5 bevatten studies over de cardiomyoplastie operaties, hoofdstukken 6,7 en 8 behandelen aortamyoplastie.

Hoofdstuk 2 behandelt een diermodel om hartvergroting te verkrijgen voor het bestuderen van de cardiomyoplastie ingreep. Als model voor deze hartvergroting is een verbinding tussen een arterie (arteria carotis) en een vene (vena jugularis) in de hals van de geit gekozen (z.g. AV-shunt). Deze ingreep veroorzaakte een stabiele en snelle hartvergroting door een verdubbeling van het hartminuutvolume.

Dit aanpassingsmechanisme van het hart zorgde ervoor dat geen tekenen van nierfalen zijn waargenomen in de studietermijn van 8 weken.

Andere belangrijke veranderingen waren te zien in het eind-diastole volume (toename) en het eind-systole volume (afname), wat resulteerde in een snelle toename van de ejectie fractie en het slagvolume. Opmerkelijk was dat er geen verandering in hartritme optrad. Direct na het maken van deze AV-shunt waren alle veranderingen nog reversibel, zoals bleek uit het tijdelijk sluiten van deze AV-shunt. Dit betekent dat de veranderingen nog binnen het werkzame deel van de Starling curve lagen.

Met echocardiografie werd vastgesteld dat de eind-diastole diameter van de linker ventrikel gedurende de 8 weken van de studie geleidelijk toenam. Deze waarnemingen werden bevestigd bij de catheterisatie op de laatste dag van de studie met de conductantie catheter. Opmerkelijk in deze studie was het uitblijven van tekenen van hartfalen en de lage mortaliteit. Daarnaast werd een significante vergroting van de linker ventrikel in alle dieren waargenomen.

In hoofdstuk 3 wordt beschreven welk hemodynamisch effect de cardiomyoplastie operatie heeft bij proefdieren met een vergroot hart. Het inbrengen van de rugspier in de borstholte verlaagt het hartminuutvolume en het linker ventrikel eind-diastole volume. Het vervolgens wikkelen van de rugspier om het hart heen, zorgde voor een extra daling van het hartminuutvolume.

Deze onderzoeksresultaten komen overeen met bevindingen in patiënten, waar in de directe postoperatieve periode ondersteuning met IABP en inotropica worden aanbevolen. Deze periode zou daarom een extra risico betekenen voor patiënten met een slechte pompfunctie. 
Hoofdstuk 4 beschrijft een studie waarbij in proefdieren een harivergroting is verkregen na 8 weken (zie hoofdstuk 2) en vervolgens een cardiomyoplastie operatie is uitgevoerd. Direct na deze operatie is de spier rond het hart gestimuleerd en het slagvolume bleek aanmerkelijk toe te nemen van $87 \pm 38 \mathrm{ml}$ tot $117 \pm 48 \mathrm{ml}$. Deze toename in slagvolume was in patiënten studies nog niet beschreven. Een mogelijke verklaring is dat deze patiënten studies pas enkele maanden na de operatie werden uitgevoerd, op een moment dat de grootte van de patiënten harten aanmerkelijk was afgenomen.

In hoofdstuk 5 is een nieuw 3 -dimensionale electroanatomische mapping systeem toegepast om de bewegingen van het hart te volgen tijdens gewone hartslagen, en bij hartslagen met een gestimuleerde spier om het hart. Het bleek dat de vermindering in linker ventrikel eind-diastole volume vooral plaats vond in het basale en middelste deel van de ventrikel.

Deze techniek maakt het mogelijk tientallen punten op de linker ventrikel binnenwand te volgen tijdens een hartslag, en zo de beweging in drie dimensies gedetailleerd te analyseren.

In hoofdstuk 6 wordt een acute studie beschreven waarin aortamyoplastie in proefdieren is uitgevoerd. De skeletspier is rond de aorta descendens gewikkeld en electrisch gestimuleerd. In vergelijking met niet gestimuleerde hartslagen was tijdens skeletspiercontracties de aortadruk hoger, en de coronaire doorbloeding verbeterd, maar er was geen daling zichtbaar van de eind-diastole bloeddruk (afterload) in de aorta. Deze positie van de skeletspier zal daarom waarschijnlijk beter geschikt zijn voor patiënten met een ischemische hartziekte.

Hoofdstuk 7 behandelt een vergelijkende studie tussen de aortamyoplastie operatie met de skeletspier om de aorta descendens gewikkeld, en de IABP techniek die als gouden standaard voor couterpulsatie therapie wordt gebruikt.

Terwijl de daling van de eind-diastole bloeddruk in de aorta alleen bij de IABP werd waargenomen, was de toename in de coronaire doorbloeding meer bij de aortamyoplastie dan bij de IABP.

In hoofdstuk 8 zijn twee operatieve mogelijkheden voor aortamyoplastie vergeleken met IABP. De twee mogelijkheden zijn het wikkelen van de skeletspier rond de aorta ascendens of rond de aorta descendens. Een toename van de coronaire doorbloeding werd niet waargenomen bij de aorta ascendens positie, maar de verlaging van de afterload was vergelijkbaar met de IABP. Deze afterload verlaging werd niet bij de aorta descendens positie gezien. Volgens deze gegevens zal de aorta ascendens positie daarom de voorkeur hebben bij patiënten in het eindstadium van hartfalen.

Met betrekking tot de gebruikte diagnostische technieken, valt te concluderen dat echografie een nuttige methode is om het hart tijdens chronische 
dierproeven te analyseren met name door zijn niet invasieve karakter. Wanneer nauwkeurige hemodynamische gegevens vereist zijn voor een studie, is deze methode echter niet voldoende objectief en nauwkeurig. Als alternatief voldoen in die studies een Swan-Ganz catheter, arteriële bloeddrukcatheters en doppler bloedstroommeters om betrouwbare metingen uit te voeren.

Als meer gedetailleerde gegevens nodig zijn om de hemodynamica en de geometrie van het hart te bestuderen, zijn de conductantie catheter en de electroanatomische mapping techniek beide goede opties, waarbij de keuze tussen deze twee afhankelijk is van de precieze vraagstelling van de studies. 


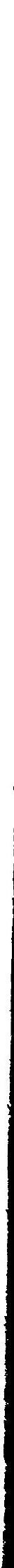




\section{Acknowledgments}

This work presents my first journey in the world of science, which started six years ago when I landed at the small airport of Maastricht (April 1995), as a young cardiothoracic resident from Israel, coming to do six months of basic science. At the airport I met for the first time Erik Van der Veen, accompanied by Kiki and Jill, his young daughters. From the airport Erik took me straight to his home were I met his wife, Astrid, and the two older daughters Nina and Lara. Dear Erik, I do believe that your kind invitation to your home as the first step of our meeting led the way to our long and close professional and personal relationship over the last six years. With this fruitful collaboration, and under your guidance in the magic roads of science, we shared many congress presentations, as well as original articles and, not less important, a lot of fun all over the world! I am sure that this thesis is just an important step in our future work together. Dear Erik, thank you from the bottom of my heart.

In Maastricht I met a group of fine people working like a super team of scientists under the directorship of Prof. Dr. HJJ Wellens who I believe is a role model for the integration between clinicians and scientists. Playing a major role in the team with his unique personality was Jan J. Schreuder. I have warm feelings and real respect for Jo Habets' and Theo Van der Nagel's professional work. The warm and professional hospitality with which I was welcomed includes so many people, of whom I would especially like to thank Birgit, Marion, Lenny and Monique.

This work should be considered as international and multi-center due to the contribution of Hans Kaulbach from Germany who helped me in my first steps in animal experimental surgery and Roberto Lorusso whose help throughout my entire work cannot be overstated. The three of us, from very different backgrounds, shared the common way of young surgeons and scientists. Hans Kaulbach, Roberto Lorusso and I spent a lot of important scientific moments together, as well as a lot of alcoholic drinks! Dear Roberto, I know you will reach the sky in your future career, but still, your strength is in your kind and warm personality, you are a real friend.

Back in Israel in 1995 I established the local group in pre clinical heart failure surgery research. Shlomo Ben-Haim, already a very busy person, supported my first steps just so that he could help a young researcher per se. The team in Israel including Edit Cohen and Tamir Wolf, was found to be very professional and under the guidance of Rona Shofti and Danyiel 
Mordohovich, made the days we spent together in the experimental surgery unit fascinating.

Several companies and their employees supported this thesis:

Telectronics - Stuart McConchie and Gordon Jacobs;

Medtronic - Pierre Grandjean;

Biosense - Yuval Sezbon, Daniel Reisfeld and Uri Yaron.

Special thanks to Max Shamgar and Rosa Boschetto from Italy for their kind support.

I would like to thank Marion Gold and Lee Cornfield for their superb editing of this work.

I met Prof. Bas. Mochtar just before he started his work in Maastricht and I appreciate his confidence in my work and his decision to choose me as the first Ph.D. in the cardiothoracic department under his directorship.

My own chief in Israel Gideon Uretzky, choose me as a medical student, raised me as a cardiac surgeon, sent me to Maastricht and later supported my research work in Israel. Gideon, you showed me the way and opened the gates. Today, together in the new department in Tel-Aviv, I feel I can start to repay you after all the years you invested in me.

Last, but not least, I want to thank my family: my parents Ada (born in Florence) and Emanuel (born in Jerusalem) and my brothers Ron and Dan for their support for so many years, for our special family spirit, and for the great Italian food.

Four years ago I met Leiku. The next day I knew I had found the love of my life. Dear Leiku, being with you helped me remember what is really important in life. Thank you for your wonderful support, thank you for Shiri and the baby on the way.

Gil 


\section{Curriculum vitae}

Born in Tel-Aviv, Israel July 14, 1960.

Technical High School, Ort Yad Singalovsky, 'Tel Aviv, 1974 - 1978.

Military Service, Jet Pilot, Major, Israeli Air-Force, 1978 - 1984.

Medical school, Ben - Gurion, Beer - Sheva, Israel. 1985- 1988.

First Year Graduated with Distinction.

Graduated, The Hebrew University - Hadassah Medical School,

Jerusalem, Israel. M. D. degree 1988 - 1991

Fifth Year Graduated with Distinction.

\section{Medical Training}

- 1992 - 1993 Internship, Sheba Medical Center, Tel Aviv, Israel.

- 1993 - Aviation Medicine, Israeli Air Force.

- 1994 - 1999 Resident at the Department of Cardiothoracic Surgery,

- Carmel Medical Center, Haifa, Israel.

- 1995 -Research fellow, Department of Cardiology, CARIM, Academic Hospital Maastricht, Maastricht, The Netherlands.

- 1997 - Thoracoscopic Surgery, European Surgical Institute, Hamburg, Germany.

- 1999 - Fellowship in Pediatric Cardiac Surgery, Marie-Lannelongue Hospital, Paris, France.

- 2000 - Consultant, Department of Cardiothoracic Surgery, Carmel Medical Center, Haifa, Israel.

- 2000 - Consultant, Department of Cardiothoracic Surgery, Tel Aviv Sourasky Medical Center, Tel Aviv, Israel.

\section{Teaching Activities: Undergraduate}

- 1986 - 1987 - Tutor in epidemiology and statistics for first year medical students at The Ben-Gurion University of the Negev Medical School.

- 1996-2000 - Tutor for sixth year medical students, Technion Medical School, Haifa, Israel, at the Department of Cardiothoracic Surgery, Carmel Medical Center, Haifa, Israel.

- 1999 - 2001 - Lecturer of the course: "Exposure to the medical system", first, second, and third year medical students, Technion Medical School, Haifa, Israel.

- 2001 - Tutor for fifth year medical students, Tel Aviv Sackler Medical School, Tel Aviv, Israel, at the Department of Cardiothoracic Surgery, Tel Aviv Sourasky Medical Center, Tel Aviv, Israel. 



\section{Publications}

\section{Articles}

1. G. Bolotin, F.H. van der Veen, J. Schreuder, R. Lorusso, J. Ben David and (i. Uretzky. "Cardiomyoplasty - the beginning of a new era" Isr I Med Sci, 1996; 32: 321-326.

2. G. Bolotin, J. Schreuder, H. Kaulbach, R. Lorusso, and F.H. van der Veen, "The Maastricht experience with the Carotid Jugular AV shunt model" Concerted Action Heart, Biomed - 1. September, 1995.

3. R. Lorusso, F.H. van der Veen, J. Schreuder, G. Bolotin, H. Kaulbach, R. Frietman, J. Habets, T. van der Nagel, H.J.J. Wellens. Hemodynamic effects in acute cardiomyoplasty of different wrapped muscle activation times as measured by pressure-volume relations. J Card Surg 1996; 1 1:217-225.

4. G.E. Amiel, R. Cohen, R. Amar, I. Sarafov, T. Safadi, M. Saute, E. Porat, R. Sharony, G. Bolotin, M. Tann, G. Uretzky. Using a video conference to teach clinical clerks in one-week cardiothoracic clerkship. Am J Surg. 1997;173:330-332.

5. T. Wolf, G. Bolotin, F.H. van der Veen, Joseph B. David, R. Lorusso, and G. Uretzky. Aortomyoplasty - a surgical treatment for end-stage heart failure. Harefuah, 1998;134(10):792-797.

6. G. Bolotin, F.H. van der Veen, R. Lorusso, J.J. Schreuder, T. Wolf, Joseph B. David and G. Uretzky. Aortomyoplasty. Basic Appl. Myol. 1998; 8(1): 59-65.

7. T. Wolf, G. Bolotin, R. Ammar and G. Uretzky. Anomalous origin of the left main coronary artery: anatomical correction and concomitant LIMA-to-LAD grafting. Eur J Cardiothorac Surg. 1999; 15: 209-212.

8. G. Bolotin, T. Wolf, F.H. van der Veen, R. Shofti, R. Lorusso, J.J. Schreuder, and G. Uretzky. Acute descending aortomyoplasty induces coronary blood flow augmentation. The Ann Thorac Surg. 1999; 68: 1668-1975.

9. G. Bolotin, R. Lorusso, H. Kaulbach, J.J. Schreuder, G. Uretzky, and F.H. van der Veen. Acute and chronic heart dilatation model - induced in goats by carotid jugular A-V shunt. Basic Appl. Myol. 1999;9(5):219-222.

10. G. Bolotin, H. Lazarovici, A Zlotnik A. Tamir and M. Saute. Efficacy of intraoperative, internal intercostal nerve block during video-assisted thoracic surgery for postoperative pain. Ann Thorac Surg 2000;70:1872-1875.

11. G. Bolotin, T. Wolf, R. Shachner, F.H. van der Veen, R. Shofi, R.Lorusso, J. J. Shreuder, G. Uretzky. Hemodynamic evaluation of descending aortomyoplasty versus intra-aortic balloon pump performed in normal animals: an acute study. Eur J Cardiothorac Surg $2001 ; 19: 174-178$ 


\section{Abstracts}

1. G. Bolotin, J. Schreuder, H, Katulbach, R. Lorusso, and I:II. van der Veen, "The Maastricht experience with the Carotid Jugular $\Lambda V$ shunt model" Biomed - 1, Proceedings of the meeting held in Florence, Italy. September 14, 1995.

2. Kaulbach HG, Van der Veen FH, Schreuder, G. Bolotin, R. Lorusso and II. J. Wellens. Dynamic Cardiomyoplasty in chronic Cardiac Overload. The 68 ammal meeting, The American Heart $\Lambda$ ssociation, 1995. Circulation , 1995; 92:I-379.

3. G. Bolotin, F.H. van der Veen, J. Schreuder, H. Kaulbach G. Uretzky and II. J. Wellens. Systolic augmentation after cardiomyoplasty in goats with ventricular dilatation. [Poster]., Israel Heart Society, Annual scientific meeting, April, 1996, Tel Aviv, Isral. Isr J Med Sci, 1996; 32 (supp): S33.

4. G. Bolotin, F.H. van der Veen, J. Schreuder, R. Lorusso, H. Kaulbach and G. Uretzky. The chronic carotid jugular $\mathrm{A}-\mathrm{V}$ shunt as a model of heart failure to study cardiomyoplasty. The Forth World Congress on Heart Failure, Jerusalem, 1996. [Abstract], The Journal of Heart Failure, 1996; 3: 591.

5. R. Lorusso, F.H. van der Veen, J. Schreuder, G. Bolotin, H. Kaulbach, J Habets, T van der Nagel, H.J.J. Wellens. Analysis of different wrapped muscle activation times by pressure/volume relation in acute cardiomyoplasty. [Poster], Cardiostim 10th International Congress, Nice, France, June 1996. The European Journal of Cardiac Pacing and Electrophysiology, 1996; 6 (supp): 152.

6. G. Bolotin, F.H. van der Veen, J. Schreuder, H. Kaulbach, G. Uretzky, IH.J.J. Wellens. Systolic augmentation after cardiomyoplasty in goats with ventricular dilatation. [Poster], Cardiostim 10th International Congress, Nice, France, June 1996. The European Journal of Cardiac Pacing and Electrophysiology, 1996; 6 (supp): 153.

7. E. Porat, A. Avineder, A. Merdler, G. Bolotin, G. Uretzky. Coronary artery fistula - surgical approach. [Film session], The European Association for Cardio-Thoracic Surgery, 10th annual meeting, Prague, Czech Republic, 1996.

8. G. Bolotin, F.H. van der Veen, J. Schreuder, R. Lorusso, H. Kaulbach, G. Uretzky. Acute temporary decrease of end diastolic volume and cardiac output following cardiomyoplasty. Mediterranean Association of Cardiology and Cardiac Surgery, 9th Annual Meeting, Tel Aviv, Israel, October 1996. Isr J Med Sci, 1996;32:911.

9. E. Porat, A. Avineder, A. Merdler, G. Bolotin, G. Uretzky. Coronary artery fistula - surgical approach. Mediterranean Association of Cardiology and Cardiac Surgery, 9th Annual Meeting, Tel Aviv, Israel. Isr J Med Sci, 1996;32:1023.

10. G. Bolotin, T. Wolf, R. Shofti, F.H. van der Veen, J.J. Schreuder, R. Lorusso, Y. Rubin and G. Uretzky. Augmentation of coronary flow following acute aortomyoplasty. The Fifth World Congress on Heart Failure, Washington, USA, 1997. The Journal of Heart Failure, 1997; 4: 744 .

11. G. Bolotin, T. Wolf, R. Shofti, F.H. van der Veen, J.J. Schreuder, R. Lorusso, Y. Rubin and G. Uretzky. Coronary flow diastolic augmentation following acute descending aortomyoplasty. International Society for Heart Research European section, Israeli Subsection. Annual meeting, May, 1997, Bar Ilan University, Israel.

12. G. Bolotin, T. Wolf, R. Shofti, F.H. van der Veen, J.J. Schreuder, R. Lorusso, Y. Rubin and G. Uretzky. Coronary flow diastolic augmentation following acute descending aortomyoplasty. The European Association for Cardio-Thoracic Surgery, 11 th annual meeting, October, 1997, Copenhagen, Denmark. 
13. G. Bolotin, I. Wolf, R. Shofti, F.H. van der Veen, M. smink, J.J. Schreuder, R. Lorusso, Y. Rubin, and G. Uretzky. Coronary blood flow augmentation and afterload reduction: $A$ comparison berween Intra-Aortic Balloon pump and descending aortomyoplasty counter-pulsation. BAM 98 - International Conference on Muscle Plasticity. Abano Terme (Padova), Italy, June 14-16, 1998. Basic and $\Lambda$ pplied Myology. 1998; 8(3): 266.

14. G. Bolotin, T. Wolf, R. Shofti, Y. Rubin, F.II. van der Veen, M. smink, J.J. Schreuder, R. Lorusso, and G. Uretzky. Descending aortomyoplasty augments coronary flow but does not produce afterload reduction: A comparison with Intra-Aortic Balloon pump. 'The European Association for Cardio-Thoracic Surgery, 12th annual meeting, October, 1999, Glasgow, Scotland.

15. G. Bolotin, H. Lazarovici, A Zlotnik A. Tamir and M. Saute. Efficacy of intraoperative, internal intercostal nerve block during video-assisted thoracic surgery for postoperative pain. $36^{\text {th }}$ Annual meeting, The Society of 'Thoracic Surgeons Fort Lauderdale, Florida, USA, February 2000.

16. G. Bolotin, T. Wolf, R. Sachner, R. Shofti, F.H. van der Veen, M. smink, R. Lorusso, and G. Uretzky. Hemodynamic analysis of descending vs. ascending aortomyoplasty, and comparison with intra-aortic balloon pump. The $47^{\text {th }}$ meeting of the Israel lleart Society, Tel- $\Lambda$ viv April 2000 . (J.J. Kellerman young investigator competition). Journal of the Israel Heart Society supp 4. April 2000.

17. G. Bolotin, R. Sachner, T. Wolf, R. Shofti, F.II. van der Veen, M. smink, R. Lorusso, and G. Uretzky. Aortic diastolic pressure augmentation integral as indicator for coronary blood flow augmentation induced by IABP. International forum: Cardiology and Cardiac Surgery into the new millennium. The $47^{\text {th }}$ meeting of the Israel Heart Society, Tel- $\Lambda$ viv April 2000. Journal of the Israel Heart Society supp 81. April 2000.

18. G. Bolotin, F.H. van der Veen, T. Wolf, R. Sachner, U. Sezbon, D. Reisfeld, R. Shofti, R. Lorusso, S. Ben-Haim, and G. Uretzky. The novel non-fluoroscopic three dimensional electroanatomical mapping system as imaging modality for the operating room of the future. The $7^{\text {th }}$ Cardiothoracic Techniques \& Technologies meeting, New Orleans, January 2001. 